\title{
The Synthesis and PKC Binding of a New Class of A-ring Diversifiable Bryostatin Analogs Utilizing a Double Asymmetric Hydrogenation and Cross-coupling Strategy
}

\author{
Paul A. Wender, ${ }^{\star}$ Joshua C. Horan \\ Department of Chemistry and Department of Molecular Pharmacology, Stanford \\ University, Stanford, CA 94305-5080
}

\author{
Supporting Information
}

\section{General experimental methods}

Air and moisture sensitive reactions were carried out in oven-dried $\left(>110{ }^{\circ} \mathrm{C}\right)$ glassware sealed with rubber septa under a positive pressure of dry nitrogen or argon from a manifold. Stirring was effected with Teflon ${ }^{\circledR}$-coated, oven-dried $\left(>110^{\circ} \mathrm{C}\right)$ magnetic stir bars cooled under a positive pressure of dry nitrogen. Air and moisture sensitive liquids and solutions were transferred via syringe or stainless steel cannula under nitrogen atmosphere.

Reaction temperatures refer to the external or bath temperature in which the reaction vessel was partially immersed. Room temperature indicates an external temperature of $20-25{ }^{\circ} \mathrm{C}$. Elevated temperatures were maintained using a silicon oil bath, which was either equilibrated to the desired temperature under constant current through a nichrome wire heating element, or actively controlled using an electronic thermostat. Temperatures of $0{ }^{\circ} \mathrm{C}$ and $-78{ }^{\circ} \mathrm{C}$ were maintained with ice/water mixtures and dry ice/acetone mixtures, respectively. "In vacuo" concentration of organic solutions refers to solvent evaporation using a rotary evaporator with a vacuum pump. Residual solvents were removed from samples using a vacuum line held at $0.1-0.5$ torr. All references to "high-vacuum" refer to a reduced pressure of $0.1-0.5$ torr.

Unless otherwise noted, all commercial reagents were used without further purification. Tetrahydrofuran, diethyl ether, toluene and dichloromethane, were passed through alumina drying columns (Solv-Tek Inc.). Anhydrous 1,4-dioxane was purchased from Aldrich Chemical Company in a Sure/Seal ${ }^{\mathrm{TM}}$ bottle. Deuterated chloroform was purchased from Cambridge Isotope Laboratories and stored over anhydrous potassium carbonate.

\section{Chromatographic methods}

Analytical TLC was performed with $0.25 \mathrm{~mm}$ glass-backed, silica gel $60 \mathrm{~F}_{254}$ coated plates from Merck. After development, plates were visualized using a $254 \mathrm{~nm}$ ultraviolet lamp. Plates were further visualized by treatment with solutions of $p$-anisaldehyde, potassium permanganate or ceric ammonium molybdate followed by gentle heating on a hot plate. Preparative column (flash) chromatography was performed using a forced flow of solvent through silica gel 60 (230-400 mesh, purchased from EMD Chemicals Inc.) as described by Still et al. ${ }^{1}$

Reverse phase high pressure liquid chromatography (RP-HPLC) was performed on a Varian ProStar model 320 or a Rainin HPXL HPLC system with a UV detector set at $254 \mathrm{~nm}$. Reverse phase column specifications were as follows: preparative column: Alltech Altima C18 $(10 \mu \mathrm{m})$ column (length: $250 \mathrm{~mm}$; ID: $22 \mathrm{~mm})$; semi-preparative column: Alltech Altima C18 (10 $\mu \mathrm{m})$

\footnotetext{
${ }^{1}$ Still, W. C.; Kahn, M.; Mitra, A. J. Org. Chem. 1978, 43, 2923-2925.
} 
column (length: $250 \mathrm{~mm}$; ID: $10 \mathrm{~mm}$ ); analytical column: Alltech Altima C18 (5 $\mu \mathrm{m})$ column (length: $150 \mathrm{~mm}$; ID: $4.6 \mathrm{~mm}$ ). Solvent was removed by lyophilization.

Normal phase HPLC was performed on a Hewlett Packard series 1100 HPLC system with a UV detector set to $254 \mathrm{~nm}$. Chiral separations were performed using a Chiralcel OD-H column. Achiral separations were performed using an Agilent Zorbax CN 4.6x250 mm column.

Gas chromatography (GC) was performed on a Hewlett Packard 6890/5973 GC/MS system equipped with an Alltech EC-5 (0.32x30 mm, $0.25 \mu \mathrm{m}$ film) column.

\section{Physical and spectroscopic measurements}

Melting points were determined using an Arthur H. Thomas Unimelt capillary melting point apparatus and are uncorrected. Optical rotations were measured using a JASCO DIP-360 digital polarimeter and are reported as follows: $[\alpha]_{D}^{\text {temperature }}=$ specific rotation in ${ }^{\circ}(\mathrm{c}=$ concentration in $\mathrm{g} / 100 \mathrm{~mL}$, solvent). Nuclear magnetic resonance spectra were measured on a Varian INOVA 600 $\left({ }^{1} \mathrm{H}\right.$ at $600 \mathrm{MHz},{ }^{13} \mathrm{C}$ at $\left.150 \mathrm{MHz}\right)$, Varian INOVA $500\left({ }^{1} \mathrm{H}\right.$ at $500 \mathrm{MHz},{ }^{13} \mathrm{C}$ at $\left.125 \mathrm{MHz}\right)$, Varian $\mathrm{XL}-400\left({ }^{1} \mathrm{H}\right.$ at $400 \mathrm{MHz},{ }^{13} \mathrm{C}$ at $\left.100 \mathrm{MHz}\right)$, or Varian $\mathrm{Gem}-300\left({ }^{1} \mathrm{H}\right.$ at $300 \mathrm{MHz},{ }^{13} \mathrm{C}$ at $\left.75 \mathrm{MHz}\right)$ magnetic resonance spectrometer. Data for ${ }^{1} \mathrm{H}$ NMR spectra are reported as follows: chemical shift $(\delta \mathrm{ppm})$ relative to residual solvent or TMS internal standard, integration, multiplicity $(\mathrm{s}=$ singlet, brs $=$ broad singlet, $\mathrm{d}=$ doublet, brd $=$ broad doublet, $\mathrm{t}=$ triplet, $\mathrm{q}=$ quartet, pent $=$ pentet, sept $=$ septet, $\mathrm{qd}=$ quartet of doublets, $\mathrm{dq}=$ doublet of quartets, $\mathrm{dd}=$ doublet of doublets, $\mathrm{dt}=$ doublet of triplets, ddd $=$ doublet of doublet of doublets, dddd $=$ doublet of doublet of doublet of doublets, ddt $=$ doublet of doublet of triplets, dsept $=$ doublet of septets, $\mathrm{m}=$ multiplet), enumerated coupling constants (in $\mathrm{Hz}$ ), and proton assignment. Proton assignments were made based on 2D NMR experiments including, COSY, HSQC, and HMBC data. Data for ${ }^{13} \mathrm{C}$ are reported in terms of chemical shift $(\delta \mathrm{ppm})$ relative to residual solvent peaks. Infrared spectra were recorded on a Perkin-Elmer 1600 Series Fourier transform spectrometer (FTIR), are reported in wavenumbers $\left(\mathrm{cm}^{-1}\right)$, and are externally referenced to polystyrene film $\left(1601 \mathrm{~cm}^{-1}\right)$. High-resolution mass spectra (HRMS) were recorded at the NIH regional mass spectrometry facility at the University of California, San Francisco, the high-resolution mass spectrometry facility at the University of California, Riverside, and the Vincent Coates foundation mass spectrometry laboratory at Stanford University, California. Reported mass values are within error limits of $5 \mathrm{ppm}$. Elemental analyses were performed by Desert Analytics, Tucson, Arizona. Reported atomic percentages are within error limits of $\pm 0.4 \%$. X-ray crystallographic data was acquired at the University of California, Berkeley and analyzed by Xavier Ottenwaelder at Stanford University. 


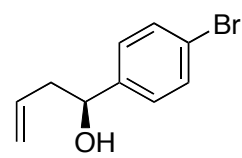

4

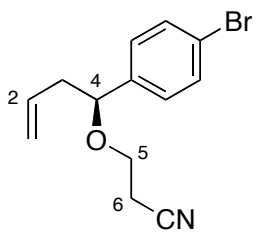

5

\author{
$\mathrm{C}_{13} \mathrm{H}_{14} \mathrm{BrNO}$ \\ Exact Mass: 279.0259 \\ Mol. Wt.: 280.1604 \\ $\mathrm{C}, 55.73 ; \mathrm{H}, 5.04 ; \mathrm{Br}, 28.52$; \\ N, $5.00 ; 0,5.71$
}

To a rt solution of alcohol $4(4.411 \mathrm{~g}, 19.42 \mathrm{mmol}$, prepared according to the method of Hanawa et al. $)^{2}$ in $\mathrm{CH}_{2} \mathrm{Cl}_{2}(39 \mathrm{~mL})$ was added acrylonitrile $(6.4 \mathrm{~mL}, 97 \mathrm{mmol})$ and Triton $\mathrm{B}(40 \%$ wt in $\mathrm{H}_{2} \mathrm{O}, 0.76 \mathrm{~mL}, 1.9 \mathrm{mmol}$ ) in separate single portions via syringe. The yellow solution was stirred for $2 \mathrm{~h}$ then poured into sat. aq. $\mathrm{NH}_{4} \mathrm{Cl}(100 \mathrm{~mL})$. The layers were separated and the aqueous layer was extracted with $\mathrm{CH}_{2} \mathrm{Cl}_{2}(3 \times 50 \mathrm{~mL})$. The combined organic layers were dried with anhydrous $\mathrm{Na}_{2} \mathrm{SO}_{4}$, filtered and concentrated in vacuo to give a brown oil. The oil was chromatographed on silica (15\% EtOAc / pentane) to give $5.203 \mathrm{~g}(96 \%)$ of the nitrile $\mathbf{5}$ as a pale yellow oil ( $92.4 \%$ ee by chiral HPLC analysis). Upon standing neat in the freezer $\left(-20{ }^{\circ} \mathrm{C}\right)$ for one week, the oil solidified into a white crystalline solid.

Recrystallization procedure: A portion of nitrile $5(711.8 \mathrm{mg})$ was suspended in $\mathrm{Et}_{2} \mathrm{O}(1 \mathrm{~mL})$ and pentane $(2.5 \mathrm{~mL})$ and warmed until all of the solids had dissolved. The solution was revoved from the heat source and one small seed crystal $(<1 \mathrm{mg})$ was added. The solution was covered and allowed to sit at $\mathrm{rt}$ for $20 \mathrm{~h}$, over which time crystal growth occurred. The supernatant was removed via pipette and $29 \% \mathrm{Et}_{2} \mathrm{O}$ /pentane $(0.5 \mathrm{~mL})$ was added to the crystals. This liquid was removed via pipette and combined with the original supernatant. The remaining crystals were dried under high-vacuum yielding $340.7 \mathrm{mg}$ of $\mathbf{5}$ as large, colorless needles ( $>99.9 \%$ ee by chiral HPLC).

The combined supernatant was concentrated in vacuo. The resulting solids were suspended in $\mathrm{Et}_{2} \mathrm{O}(0.5 \mathrm{~mL})$ and pentane $(1.25 \mathrm{~mL})$ and warmed until all of the solids had dissolved. The solution was revoved from the heat source and a $2.1 \mathrm{mg}$ seed crystal from the first crop was added. The solution was covered and allowed to sit at rt overnight, over which time crystal growth occurred. The supernatant was removed via pipette and the crystals were rinsed with $29 \%$ $\mathrm{Et}_{2} \mathrm{O} /$ pentane $(0.5 \times 2 \mathrm{~mL})$. The crystals were dried under high-vacuum yielding $209.5 \mathrm{mg}$ of $\mathbf{5}$ as large, colorless needles ( $>99.9 \%$ ee by chiral HPLC).

The combined supernatant was concentrated in vacuo. The resulting solids were were suspended in $\mathrm{Et}_{2} \mathrm{O}(0.25 \mathrm{~mL})$ and pentane $(0.63 \mathrm{~mL})$ and warmed until all of the solids had dissolved. The solution was revoved from the heat source and a $2.5 \mathrm{mg}$ seed crystal from the previous crop was added. The solution was covered and allowed to sit at $\mathrm{rt}$ for $27 \mathrm{~h}$, over which time crystal growth occurred. The supernatant was removed via pipette and the crystals were rinsed with $29 \% \mathrm{Et}_{2} \mathrm{O}$ /pentane $(0.25 \times 2 \mathrm{~mL})$. The crystals were dried under high-vacuum yielding $30.6 \mathrm{mg}$ of $\mathbf{5}$ as a single, colorless needle ( $99.4 \%$ ee by chiral HPLC).

The combined supernatant was concentrated in vacuo. The resulting solids were were suspended in $\mathrm{Et}_{2} \mathrm{O}(0.07 \mathrm{~mL})$ and pentane $(0.20 \mathrm{~mL})$ and warmed until all of the solids had dissolved. The solution was revoved from the heat source and a $3.0 \mathrm{mg}$ seed crystal from the previous crop was added. The solution was covered and allowed to sit overnight, over which time crystal growth occurred. The flask was uncovered and allowed to sit in a fume hood until the total liquid volume was reduced by $50 \%$. The supernatant was removed via pipette and the crystals were rinsed with $30 \% \mathrm{Et}_{2} \mathrm{O} /$ pentane $(0.1 \times 3 \mathrm{~mL})$. The crystals were dried under high-vacuum yielding $25.8 \mathrm{mg}$ of $\mathbf{5}$ as colorless needles (99.6\% ee by chiral HPLC). In total, $599 \mathrm{mg}$ of $\mathbf{5}$ ( $>99.8 \%$ ee) was recovered ( $84 \%$ recrystallization yield).

\footnotetext{
${ }^{2}$ Hanawa, H.; Hashimoto, T.; Maruoka, K. J. Am. Chem. Soc. 2003, 125, 1708-1709.
} 
Data for 5 :

$\mathbf{R}_{f}=0.39$ (15\% EtOAc / pentane $)-$ one black spot w/ p-anisaldehyde stain.

MP: $49.0-50.0^{\circ} \mathrm{C}$.

IR (thin film): 3077, 2876, 2252, 1906, 1839, 1792, 1641, 1592, 1486, 1409, 1394, 1339, 1297 , $1277,1221,1104,1070,1009,917,821 \mathrm{~cm}^{-1}$.

${ }^{1}$ HNMR (400 MHz, CDCl $)_{3}$ : $\delta 7.49$ (2H, m, ArH), $7.18(2 \mathrm{H}, \mathrm{m}, \mathrm{ArH}), 5.75$ (1H, m, C2), 5.04 $(2 \mathrm{H}, \mathrm{m}, \mathrm{C} 1), 4.28(1 \mathrm{H}, \mathrm{dd}, J=6.0,7.5 \mathrm{~Hz}, \mathrm{C} 4), 3.51(1 \mathrm{H}, \mathrm{dt}, J=9.3,6.3 \mathrm{~Hz}, \mathrm{C} 5), 3.48(1 \mathrm{H}, \mathrm{dt}, J$ $=9.3,6.3 \mathrm{~Hz}, \mathrm{C} 5), 2.56(3 \mathrm{H}, \mathrm{m}, \mathrm{C} 3 \mathrm{\&} \mathrm{C} 6), 2.38(1 \mathrm{H}, \mathrm{dddt}, J=6.0,7.2,14.4,1.2 \mathrm{~Hz}, \mathrm{C} 3)$.

${ }^{13}$ CNMR (100 MHz, $\mathrm{CDCl}_{3}$ ): $\delta$ 140.0, 133.7, 131.7 (2C), 128.3 (2C), 121.8, 117.74, 117.72, 82.1, $63.3,42.2,19.0$.

HRMS (DCI): Calculated for $\mathrm{C}_{13} \mathrm{H}_{18} \mathrm{BrN}_{2} \mathrm{O}$ [M+NH 4 ]: 297.0603. Found: 297.0603.

Elemental Analysis: Calculated for $\mathrm{C}_{13} \mathrm{H}_{14} \mathrm{BrNO}$ : C 55.73; H 5.04; N 5.00; Found: C 55.96; H $5.35 ; \mathrm{N} 4.78$.

$[\alpha]_{D}^{23}=-44.3^{\circ}\left(c=1.4, \mathrm{CHCl}_{3}\right)$.

X-Ray Crystal ORTEP:

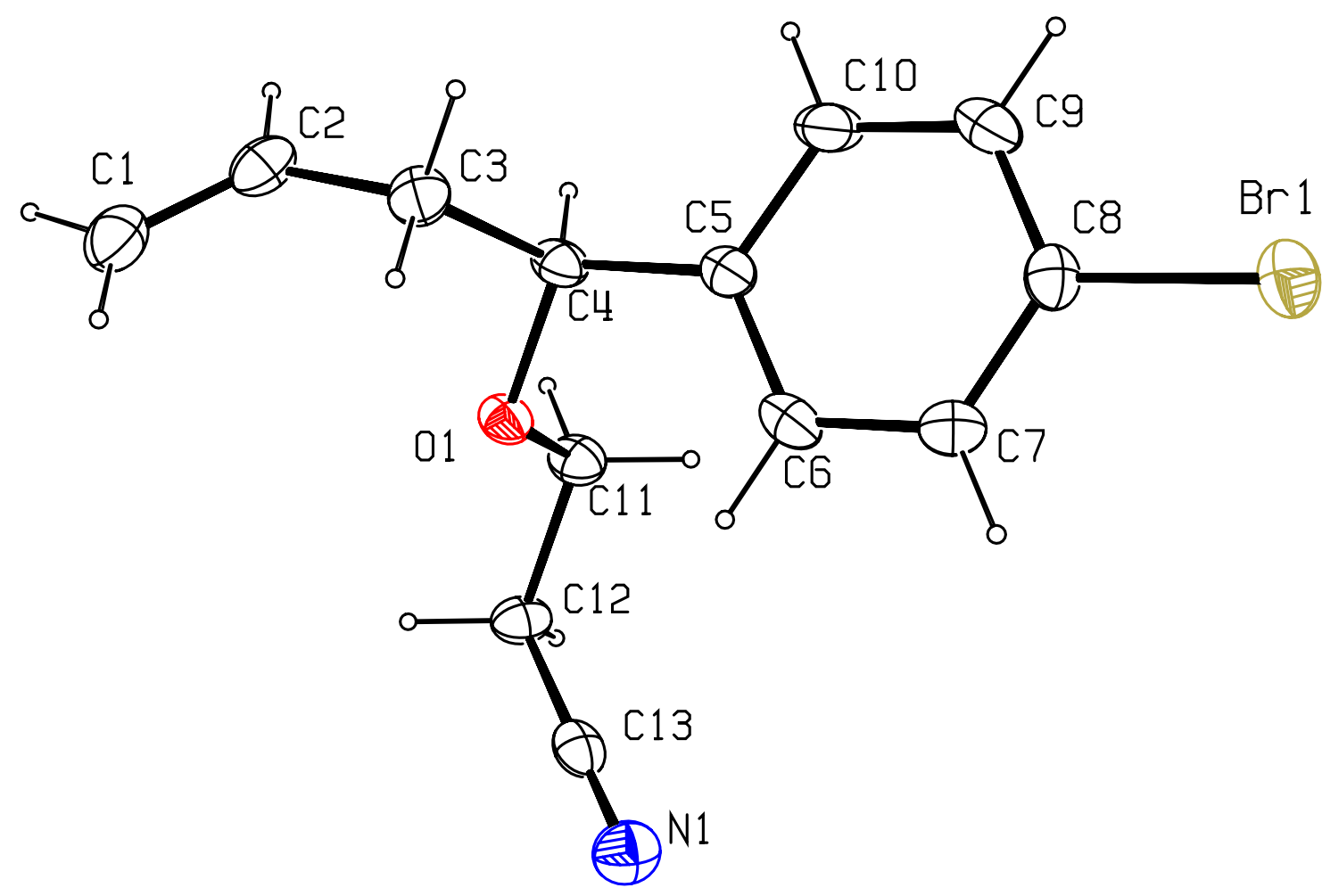



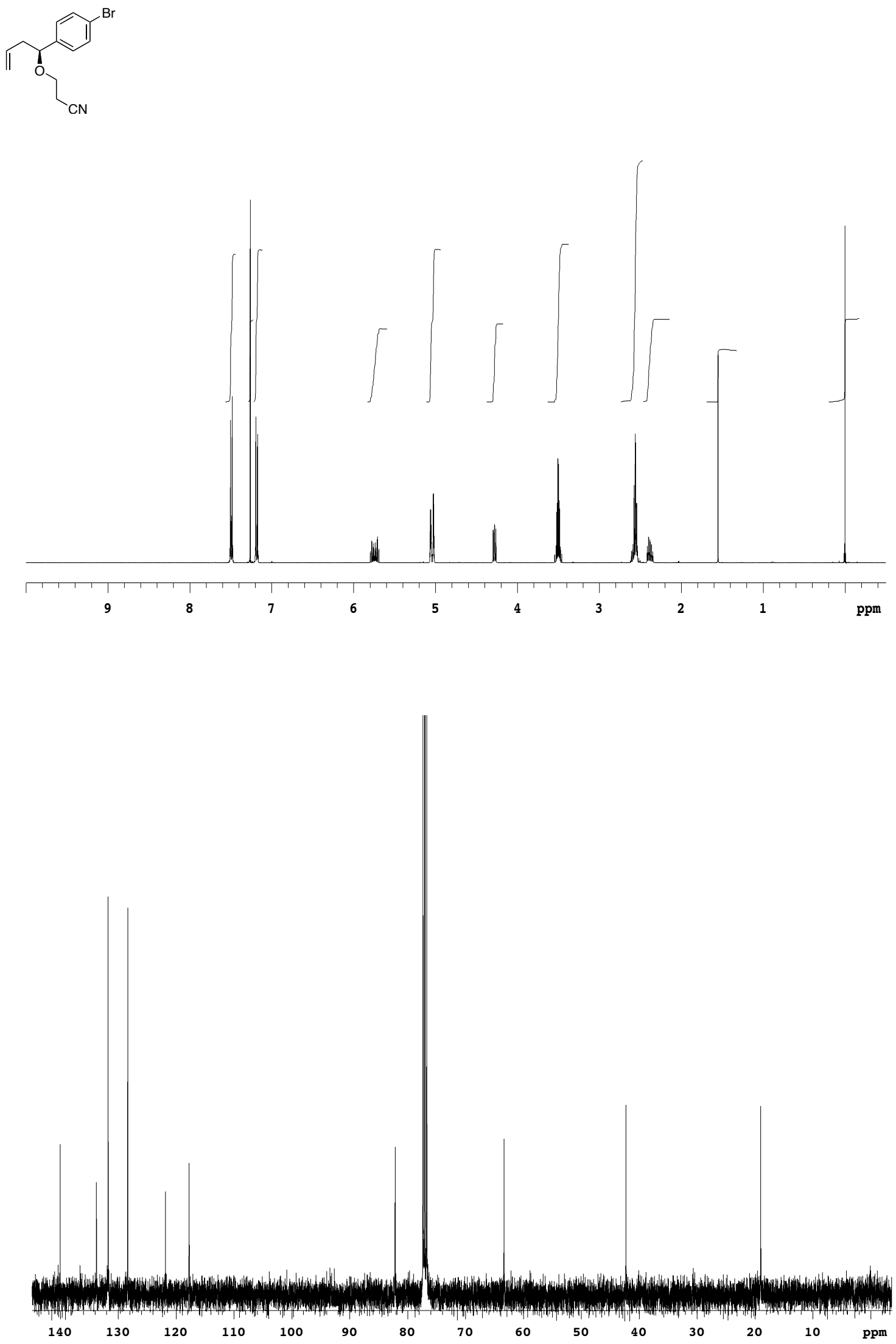

S-5 
Normal Phase HPLC for 5: chiral column; isocratic $10 \% i$-PrOH / hexanes.

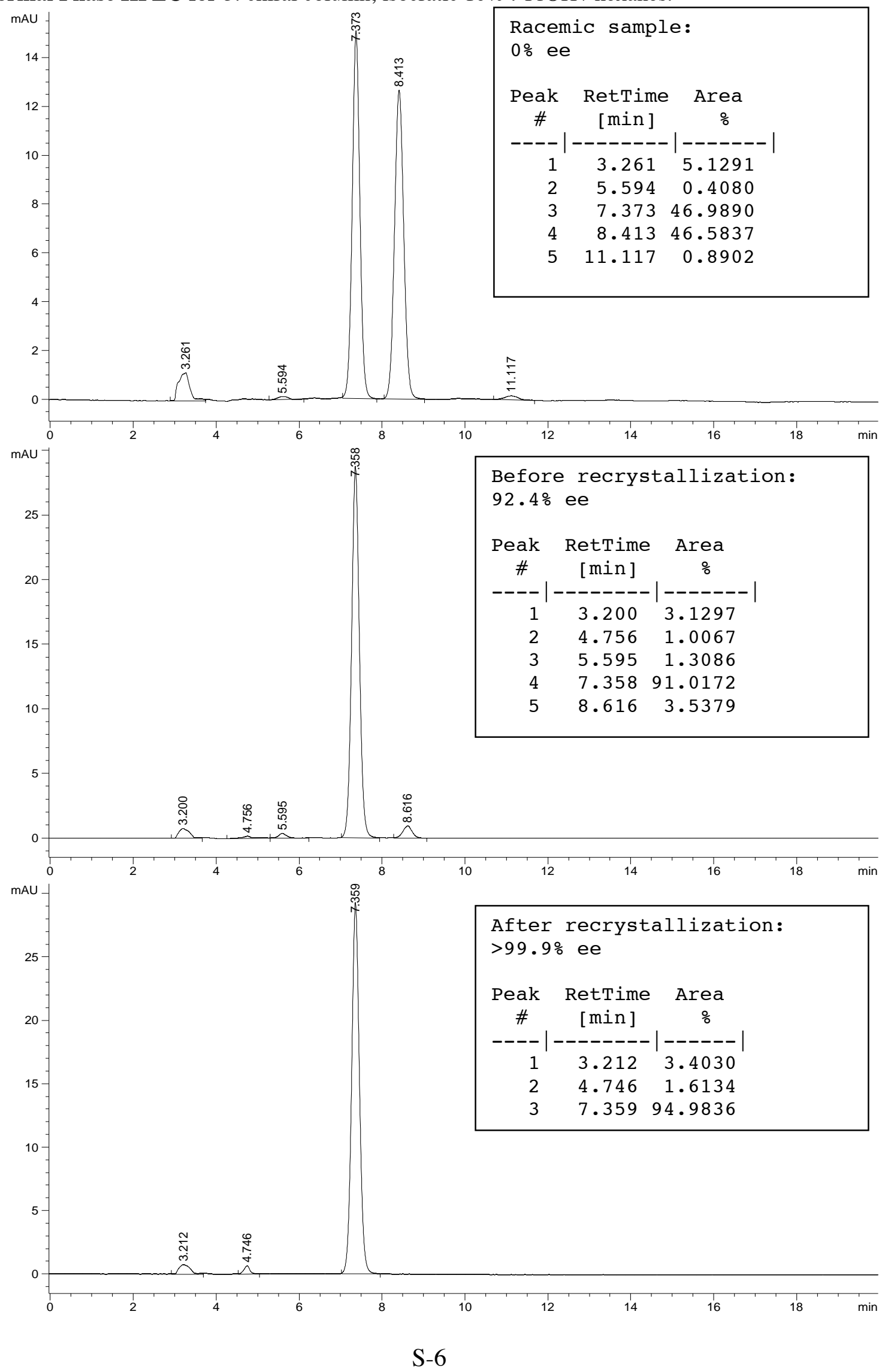




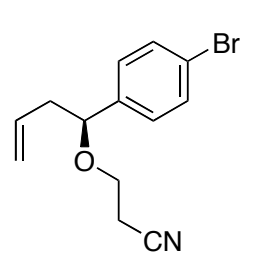

5

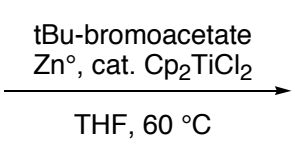

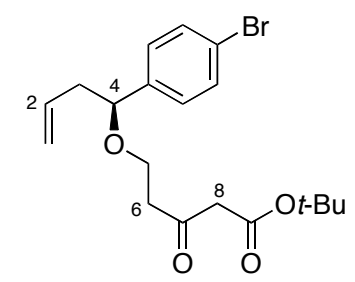

6

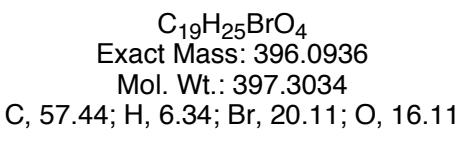

Exact Mass: 396.0936

Nitrile 5 (0.680 g, $2.43 \mathrm{mmol})$, zinc dust (2.40 g, $36.4 \mathrm{mmol})$ and $\mathrm{Cp}_{2} \mathrm{TiCl}_{2}(18.1 \mathrm{mg}, 0.0728$ mmol) were measured into a dry flask at rt. To this solid mixture was added THF $(12 \mathrm{~mL})$ followed by $t$-butyl-bromoacetate ( 3 drops). The reaction flask was partially immersed in a $60{ }^{\circ} \mathrm{C}$ oil bath and $t$-butyl-bromoacetate $(3.30 \mathrm{~mL}, 24.3 \mathrm{mmol})$ was added via syringe portionwise over $3 \mathrm{~h}$. The reaction was stirred for an additional $1 \mathrm{~h}$ at $60{ }^{\circ} \mathrm{C}$ and then allowed to cool to rt. The green suspension was filtered through a pad of celite using $\mathrm{Et}_{2} \mathrm{O}$. Aqueous $1 \mathrm{~N} \mathrm{HCl}(25 \mathrm{~mL})$ was added to the filtrate (a white precipitate initially formed and then slowly dissolved) and the biphasic mixture was stirred for $3 \mathrm{~h}$. The layers were separated and the aqueous layer was extracted $3 \times 30 \mathrm{~mL}$ with $\mathrm{Et}_{2} \mathrm{O}$. The combined organic layers were dried with anhydrous $\mathrm{MgSO}_{4}$, filtered and concentrated in vacuo to give a brown oil. The oil was chromatographed on silica (10\% EtOAc / pentane) to give $926.4 \mathrm{mg}(96 \%)$ of $\beta$-ketoester $\mathbf{6}$ as a pale yellow liquid.

Data for 6 :

$\mathbf{R}_{f}=0.56$ (15\% EtOAc / pentane) - one black/red spot w/ p-anisaldehyde stain.

IR (thin film): 3077, 2979, 2933, 2873, 1716, 1642, 1592, 1485, 1393, 1368, 1315, 1253, 1148, $1094,1071,1010,951,918,823 \mathrm{~cm}^{-1}$.

${ }^{1}$ HNMR (500 MHz, CDCl $)_{3}$ ): $7.46(2 \mathrm{H}, \mathrm{m}, \operatorname{ArH}), 7.15(2 \mathrm{H}, \mathrm{m}, \operatorname{ArH}), 5.70(1 \mathrm{H}, \mathrm{m}, \mathrm{C} 2), 5.00$ $(2 \mathrm{H}, \mathrm{m}, \mathrm{C} 1), 4.22(1 \mathrm{H}, \mathrm{dd}, J=6.1,7.5 \mathrm{~Hz}, \mathrm{C} 4), 3.55(2 \mathrm{H}, \mathrm{dt}, J=1.9,6.1 \mathrm{~Hz}, \mathrm{C} 5), 3.39(2 \mathrm{H}, \mathrm{s}$, C8), $2.77(1 \mathrm{H}, \mathrm{dt}, J=16.7,6.4 \mathrm{~Hz}, \mathrm{C} 6), 2.72(1 \mathrm{H}, \mathrm{dt}, J=16.7,6.1 \mathrm{~Hz}, \mathrm{C} 6), 2.50(1 \mathrm{H}, \mathrm{dddt}, J=$ 6.9, 7.4, 14.2, $1.3 \mathrm{~Hz}, \mathrm{C} 3), 2.33(1 \mathrm{H}, \mathrm{dddt}, J=5.9,7.1,14.2,1.3 \mathrm{~Hz}, \mathrm{C} 3), 1.47(9 \mathrm{H}, \mathrm{s}, \mathrm{t}-\mathrm{Bu})$; Note: spectrum shows $\sim 10 \%$ of the enol tautomer present in solution.

${ }^{13}$ CNMR (125 MHz, $\mathrm{CDCl}_{3}$ ): $\delta$ 201.7, 166.2, 140.7, 134.1, 131.5 (2C), 128.3 (2C), 121.4, 117.3, $81.9,81.8,63.7,51.1,42.9,42.3,27.9(3 \mathrm{C})$.

HRMS (DCI): Calculated for $\mathrm{C}_{19} \mathrm{H}_{29} \mathrm{BrNO}_{4}\left[\mathrm{M}+\mathrm{NH}_{4}\right]$ : 414.1280. Found: 414.1275.

Elemental Analysis: Calculated for $\mathrm{C}_{19} \mathrm{H}_{25} \mathrm{BrO}_{4}$ : C 57.44; H 6.34. Found: C 57.23; H 6.16.

$$
[\alpha]_{D}^{23}=-11.3^{\circ}\left(c=2.33, \mathrm{C}_{6} \mathrm{H}_{6}\right)
$$



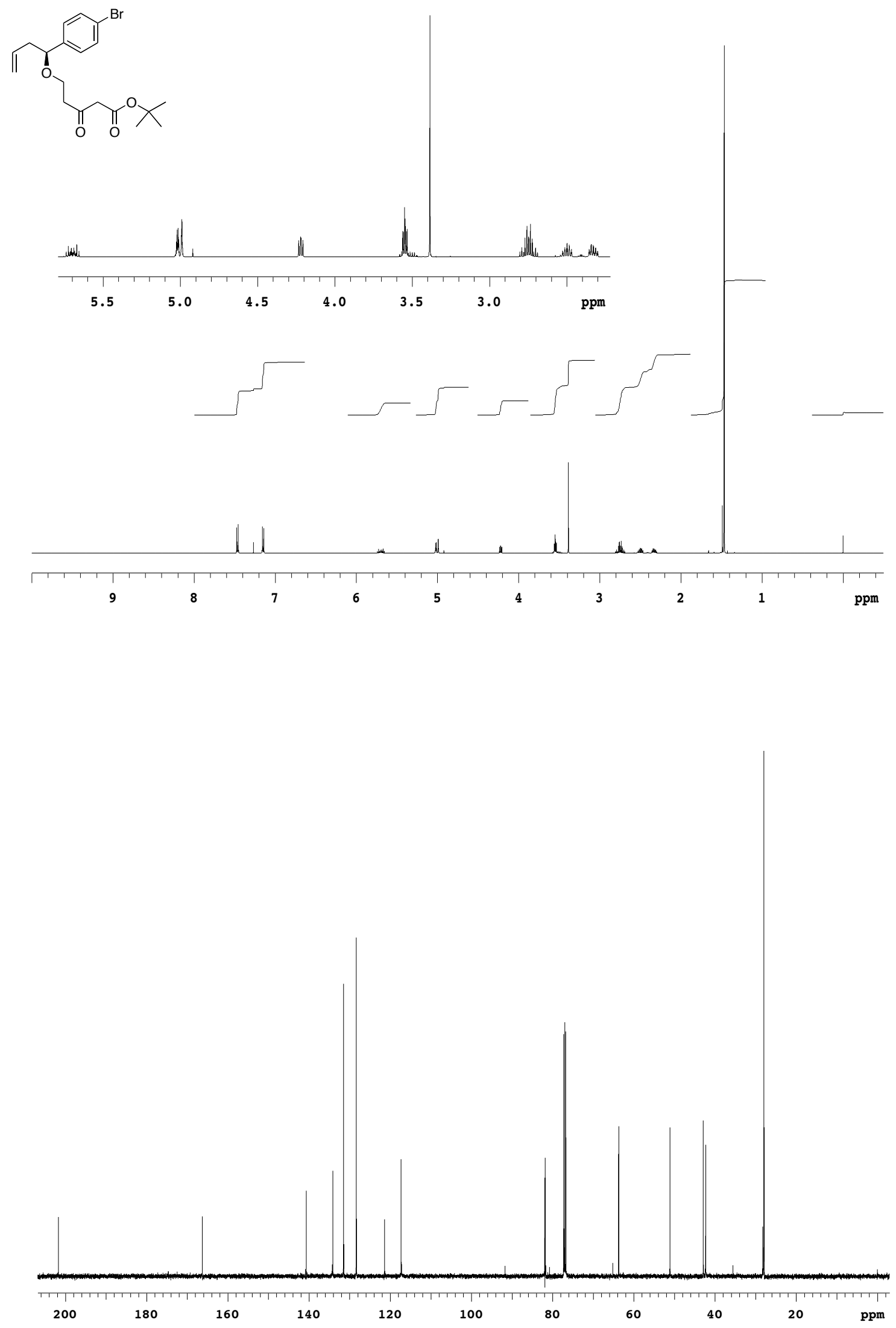


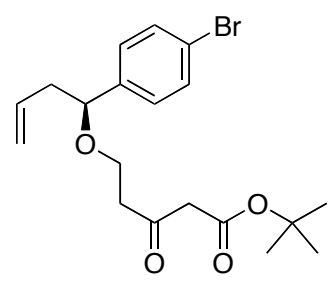

6 a. $\mathrm{O}_{3}, \mathrm{CH}_{2} \mathrm{Cl}_{2},-78^{\circ} \mathrm{C}$ b. $\mathrm{PPh}_{3},-78^{\circ} \mathrm{C} \rightarrow \mathrm{rt}$ c. ethyldiazoacetate
$\mathrm{SnCl}_{4},-78^{\circ} \mathrm{C} \rightarrow \mathrm{rt}$

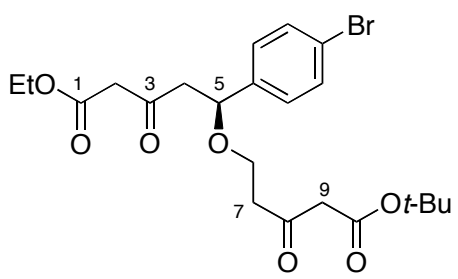

7
$\mathrm{C}_{22} \mathrm{H}_{29} \mathrm{BrO}_{7}$

Exact Mass: 484.1097

Mol. Wt.: 485.3655

C, 54.44; $\mathrm{H}, 6.02$;

$\mathrm{Br}, 16.46 ; \mathrm{O}, 23.07$

Ozone was bubbled through a solution of olefin 6 (2.05 g, $5.16 \mathrm{mmol})$ in $\mathrm{CH}_{2} \mathrm{Cl}_{2}(57 \mathrm{~mL})$ at $-78^{\circ} \mathrm{C}$ (bath temp) until a blue color persisted $(5 \mathrm{~min})$. Oxygen gas was then bubbled through the solution until the blue color disappeared $(7 \mathrm{~min})$. To the cooled solution was added solid triphenylphosphine $(1.42 \mathrm{~g}, 5.41 \mathrm{mmol})$ in one portion. After removal of the cold bath, the flask was allowed to warm to $\mathrm{rt}$ and the solution was stirred for $2 \mathrm{~h}$ (the phosphine did not completely dissolve until the temperature reached $\mathrm{rt}$ ). The reaction flask was reimmersed in the $-78{ }^{\circ} \mathrm{C}$ bath and ethyl diazoacetate $(2.02 \mathrm{~mL}, 15.5 \mathrm{mmol})$ was added as a single portion via syringe. Following this, $\mathrm{SnCl}_{4}\left(1 \mathrm{M}\right.$ in $\left.\mathrm{CH}_{2} \mathrm{Cl}_{2}, 2.58 \mathrm{~mL}, 2.58 \mathrm{mmol}\right)$ was added via syringe in a single portion. The bath was allowed to warm to $-65^{\circ} \mathrm{C}$ over $20 \mathrm{~min}$, then the flask was transferred to a $-20{ }^{\circ} \mathrm{C}$ bath. The bath was allowed to gradually warm to $-10{ }^{\circ} \mathrm{C}$ over $1 \mathrm{~h}$ and then to $5{ }^{\circ} \mathrm{C}$ over 1 additional $\mathrm{h}$. The bath was removed and the flask was allowed to warm to $\mathrm{rt}$ for the last $1 \mathrm{~h}$ of stirring. The reaction was poured into a mixture of sat aq. $\mathrm{NaHCO}_{3}(20 \mathrm{~mL})$ and $\mathrm{H}_{2} \mathrm{O}(20 \mathrm{~mL})$. The layers were separated and the aqueous layer was extracted with $\mathrm{CH}_{2} \mathrm{Cl}_{2}(2 \times 75 \mathrm{~mL})$. The combined organic layers were dried with anhydrous $\mathrm{MgSO}_{4}$, filtered and concentrated in vacuo (a white precipitate formed upon concentration). The resulting liquid mixture was chromatographed on silica $(20 \rightarrow 35 \%$ EtOAc / pentane) to give $1.8219 \mathrm{~g}(73 \%)$ of the di- $\beta$-ketoester 7 as a pale yellow oil.

Data for 7:

$\mathbf{R}_{f}=0.47$ (30\% EtOAc / pentane) - one red spot w/ p-anisaldehyde stain.

IR (thin film): 2979, 2933, 1718, 1643, 1485, 1395, 1368, 1319, 1252, 1148, 1096, 1032, 1010, $948,826 \mathrm{~cm}^{-1}$.

${ }^{1}$ HNMR $\left(500 \mathrm{MHz}, \mathrm{CDCl}_{3}\right): \delta 7.48(2 \mathrm{H}, \mathrm{m}, \mathrm{ArH}), 7.19(2 \mathrm{H}, \mathrm{m}, \mathrm{ArH}), 4.73(1 \mathrm{H}, \mathrm{dd}, J=4.3,9.1$ $\mathrm{Hz}, \mathrm{C} 5), 4.17(2 \mathrm{H}, \mathrm{q}, J=7.2 \mathrm{~Hz}, \mathrm{Et}), 3.58(1 \mathrm{H}, \mathrm{ddd}, J=5.9,6.6,9.6 \mathrm{~Hz}, \mathrm{C} 6), 3.53(1 \mathrm{H}, \mathrm{ddd}, J=$ $5.6,6.4,9.6 \mathrm{~Hz}, \mathrm{C} 6), 3.44(2 \mathrm{H}, \mathrm{d}, J=1.2, \mathrm{C} 2$ or C9), $3.36(2 \mathrm{H}, \mathrm{d}, J=3.9, \mathrm{C} 2$ or C9), $3.01(1 \mathrm{H}$, $\mathrm{dd}, J=9.0,16.4 \mathrm{~Hz}, \mathrm{C} 4), 2.74(2 \mathrm{H}, \mathrm{dt}, J=5.7,6.7 \mathrm{~Hz}, \mathrm{C} 7), 2.67(1 \mathrm{H}, \mathrm{dd}, J=4.2,16.4 \mathrm{~Hz}, \mathrm{C} 4)$, $1.46(9 \mathrm{H}, \mathrm{s}, \mathrm{t}-\mathrm{Bu}), 1.26(3 \mathrm{H}, \mathrm{t}, J=7.2$, Et); Note: spectrum shows $>13 \%$ of enol tautomers present in solution.

${ }^{13}$ CNMR $\left(125 \mathrm{MHz}, \mathrm{CDCl}_{3}\right): \delta$ 201.4, 200.3, 166.8, 166.2, 139.6, 131.8 (2C), 128.2 (2C), 121.9, 82.0, 77.4, 63.7, 61.4, 50.9, 50.8, 50.1, 42.7, 27.9 (3C), 14.1.

HRMS (EI+): Calculated for $\mathrm{C}_{18} \mathrm{H}_{20} \mathrm{BrO}_{7}\left[\mathrm{M}^{+}-t \mathrm{Bu}\right]: 427.0392$. Found: 427.0410.

Elemental Analysis: Calculated for $\mathrm{C}_{22} \mathrm{H}_{29} \mathrm{BrO}_{7}$ : C 54.44; H 6.02. Found: C 54.60; H 6.31.

$[\alpha]_{D}^{23}=-23.8^{\circ}\left(c=4.12, \mathrm{C}_{6} \mathrm{H}_{6}\right)$. 

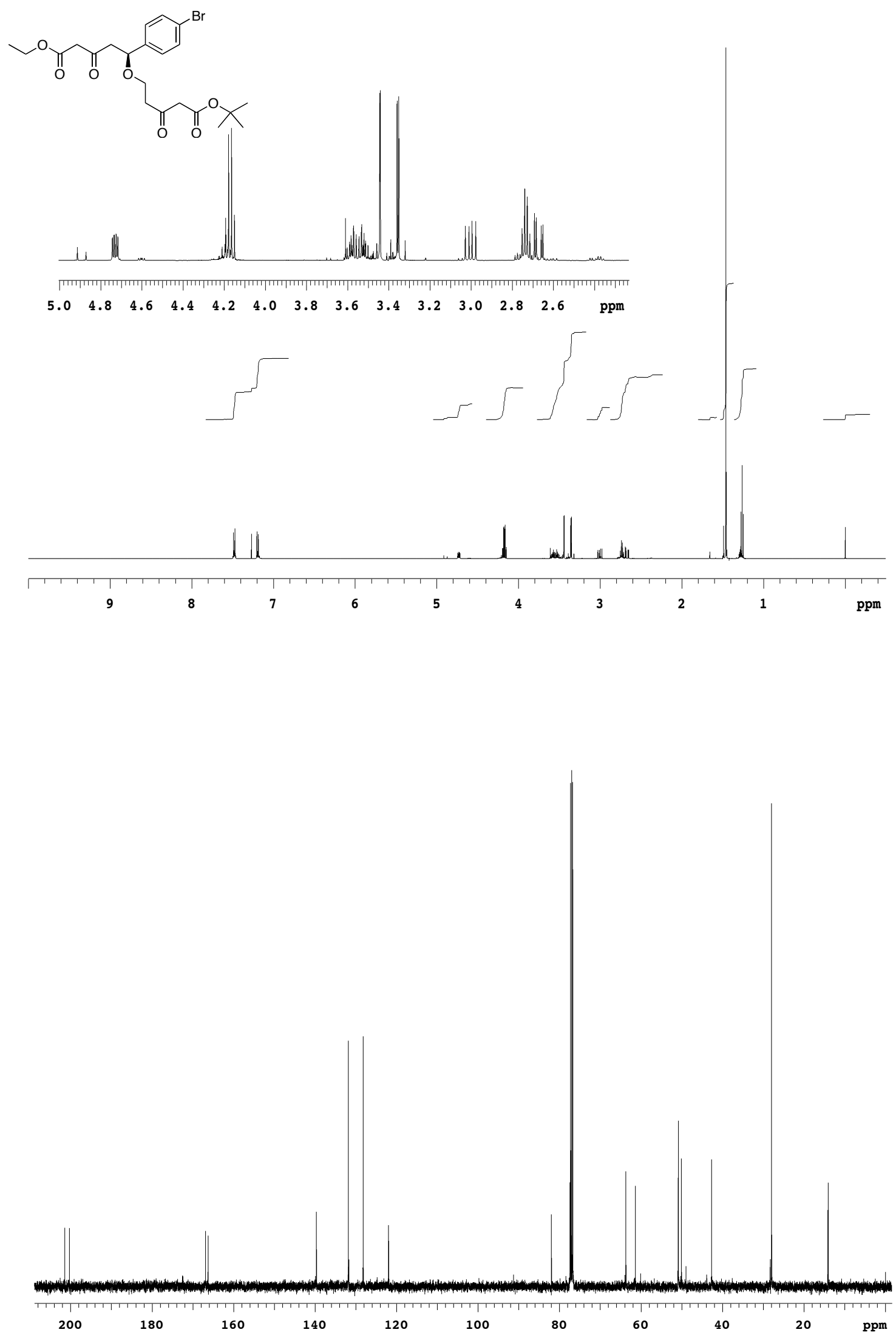

S-10 


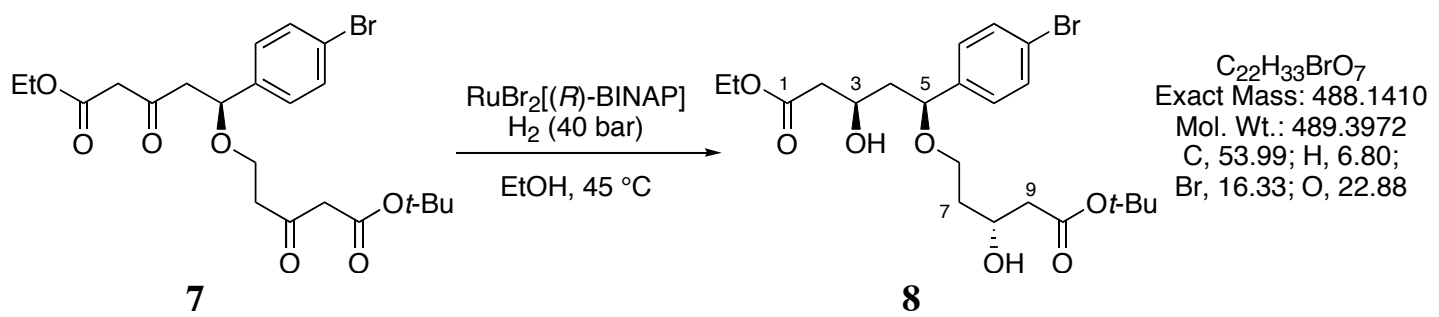

The catalyst was prepared in a manner similar to the procedure used by Blanc et $a l^{3}(R)$ BINAP $(34.7 \mathrm{mg}, 55.7 \mu \mathrm{mol})$ and (COD)Ru(2-methylallyl) $)_{2}(14.8 \mathrm{mg}, 46.4 \mu \mathrm{mol}$, Acros) were placed into a dry flask under $\mathrm{N}_{2}$ and degassed anhydrous acetone $(6.5 \mathrm{~mL})$ was added. A methanolic solution of $\mathrm{HBr}(11.3 \mu \mathrm{L}$ of $49 \%$ aq. $\mathrm{HBr}$ in $564 \mu \mathrm{L}$ degassed $\mathrm{MeOH}, 0.102 \mathrm{mmol})$ was added to the resulting suspension in one portion via syringe and the red mixture was stirred at $\mathrm{rt}$ for $50 \mathrm{~min}$. The solvent was reduced by $75 \%$ under a stream of $\mathrm{N}_{2}$ gas and then concentrated to dryness in vacuo. The resulting red/brown solid was placed under high-vacuum for $45 \mathrm{~min}$ and used directly as the catalyst for the hydrogenation reaction.

To the prepared catalyst was added di- $\beta$-ketoester $7(450 \mathrm{mg}, 0.927 \mathrm{mmol})$ as a solution in degassed EtOH (2.0 mL, 200 proof, Gold Shield Chemical Company) and the reaction flask was placed in a high-pressure bomb, flushed with $\mathrm{N}_{2}$ and sealed. The apparatus was pressurized and purged with $\mathrm{H}_{2}(20 \mathrm{bar})$ three times and then finally pressurized to $40 \mathrm{bar} \mathrm{H}_{2}$. The apparatus was placed in a $45{ }^{\circ} \mathrm{C}$ oil bath on a stir plate and allowed to proceed for $72 \mathrm{~h}$. The apparatus was removed from the bath, allowed to cool to $\mathrm{rt}$ and then slowly depressurized. The solvent was removed in vacuo and the brown residue was chromatographed on silica (45\% EtOAc / pentane) to give $419.5 \mathrm{mg}$ of a viscous, pale green liquid. HPLC analysis of this mixture revealed it to be a 97.8:1.4:0.7:0.1 mixture of the four possible diastereomers. The major diastereomer was separated from the mixture using the same chromatography conditions listed above to give 391.7 $\mathrm{mg}$ of $\mathbf{8}$ as a colorless oil ( $>99 \%$ de, $89 \%)$.

Non-selective reduction (prepared for comparative purposes): To a solution of di- $\beta$-ketoester $7(39 \mathrm{mg}, 0.081 \mathrm{mmol})$ in $\mathrm{MeOH}(1.0 \mathrm{~mL})$ in a $0{ }^{\circ} \mathrm{C}$ ice bath was added $\mathrm{NaBH}_{4}(5.5 \mathrm{mg}, 0.15$ mmol). The reaction was stirred for $20 \mathrm{~min}$ at $0^{\circ} \mathrm{C}$ then sat. aq. $\mathrm{NH}_{4} \mathrm{Cl}(1.5 \mathrm{~mL})$ was added and the mixture was extracted with EtOAc $(3 \times 3 \mathrm{~mL})$. The combined organic layers were dried over $\mathrm{MgSO}_{4}$ and concentrated. Flash chromatography (silica, 40\% EtOAc / pentane) provided $30 \mathrm{mg}$ of a mixture of diastereomers as a colorless, viscous oil.

Data for 8:

$\mathbf{R}_{f}=0.26$ (40\% EtOAc / pentane $)$ - one black spot $\mathrm{w} / \mathrm{p}$-anisaldehyde stain.

IR (thin film): 3454 (br), 2979, 2934, 1726, 1592, 1486, 1393, 1368, 1295, 1258, 1154, 1096, $1071,1010,949,827 \mathrm{~cm}^{-1}$.

${ }^{1}$ HNMR (500 MHz, $\left.\mathrm{CDCl}_{3}\right): \delta 7.48(2 \mathrm{H}, \mathrm{m}, \mathrm{ArH}), 7.20(2 \mathrm{H}, \mathrm{m}, \mathrm{ArH}), 4.49(1 \mathrm{H}, \mathrm{dd}, J=5.6,8.3$ $\mathrm{Hz}, \mathrm{C} 5), 4.14(2 \mathrm{H}, \mathrm{dq}, J=1.5,7.2 \mathrm{~Hz}, \mathrm{Et}), 4.11(1 \mathrm{H}, \mathrm{m}, \mathrm{C} 8), 4.06(1 \mathrm{H}$, dddd, $J=2.8,4.4,8.1$, $12.3 \mathrm{~Hz}, \mathrm{C} 3), 3.70(1 \mathrm{H}$, br s, $-\mathrm{OH}), 3.48(1 \mathrm{H}, \mathrm{ddd}, J=5.5,7.0,9.4 \mathrm{~Hz}, \mathrm{C} 6), 3.41(1 \mathrm{H}, \mathrm{dt}, J=9.4$, $5.7 \mathrm{~Hz}, \mathrm{C} 6), 3.39(1 \mathrm{H}, \mathrm{br} \mathrm{s},-\mathrm{OH}), 2.50(1 \mathrm{H}, \mathrm{dd}, J=8.1,16.1, \mathrm{C} 2), 2.40(1 \mathrm{H}, \mathrm{dd}, J=4.4,16.1$, C2), $2.36(1 \mathrm{H}, \mathrm{dd}, J=4.8,16.4, \mathrm{C} 9), 2.32(1 \mathrm{H}, \mathrm{dd}, J=7.7,16.4, \mathrm{C} 9), 2.01$ (1H, ddd, $J=8.4,9.7$, $14.3 \mathrm{~Hz}, \mathrm{C} 4), 1.69$ (3H, m, C4 \& C7), 1.46 (9H, s, t-Bu), 1.25 (3H, t, $J=7.08 \mathrm{~Hz}, \mathrm{Et})$.

${ }^{13}$ CNMR (125 MHz, $\mathrm{CDCl}_{3}$ ): $\delta$ 172.2, 172.0, 140.5, 131.8 (2C), 128.3 (2C), 121.7, 81.3, 81.1, 66.8, 66.0, 65.9, 60.7, 44.3, 42.3, 41.6, 36.1, 28.1 (3C), 14.1;

\footnotetext{
${ }^{3}$ Blanc, D.; Ratovelomanana-Vidal, V.; Marinetti, A.; Genet, J. P. Synlett 1999, 480-482.
} 
HRMS (FAB+): Calculated for $\mathrm{C}_{22} \mathrm{H}_{33} \mathrm{BrO}_{7} \mathrm{Na}[\mathrm{M}+\mathrm{Na}]$ : 511.1307 . Found: 511.1313.

Elemental Analysis: Calculated for $\mathrm{C}_{22} \mathrm{H}_{33} \mathrm{BrO}_{7}$ : C 53.99; H 6.80. Found: C 53.69; H 7.19. $[\alpha]_{D}^{23}=-36.1^{\circ}\left(c=1.70, \mathrm{CH}_{2} \mathrm{Cl}_{2}\right)$. 

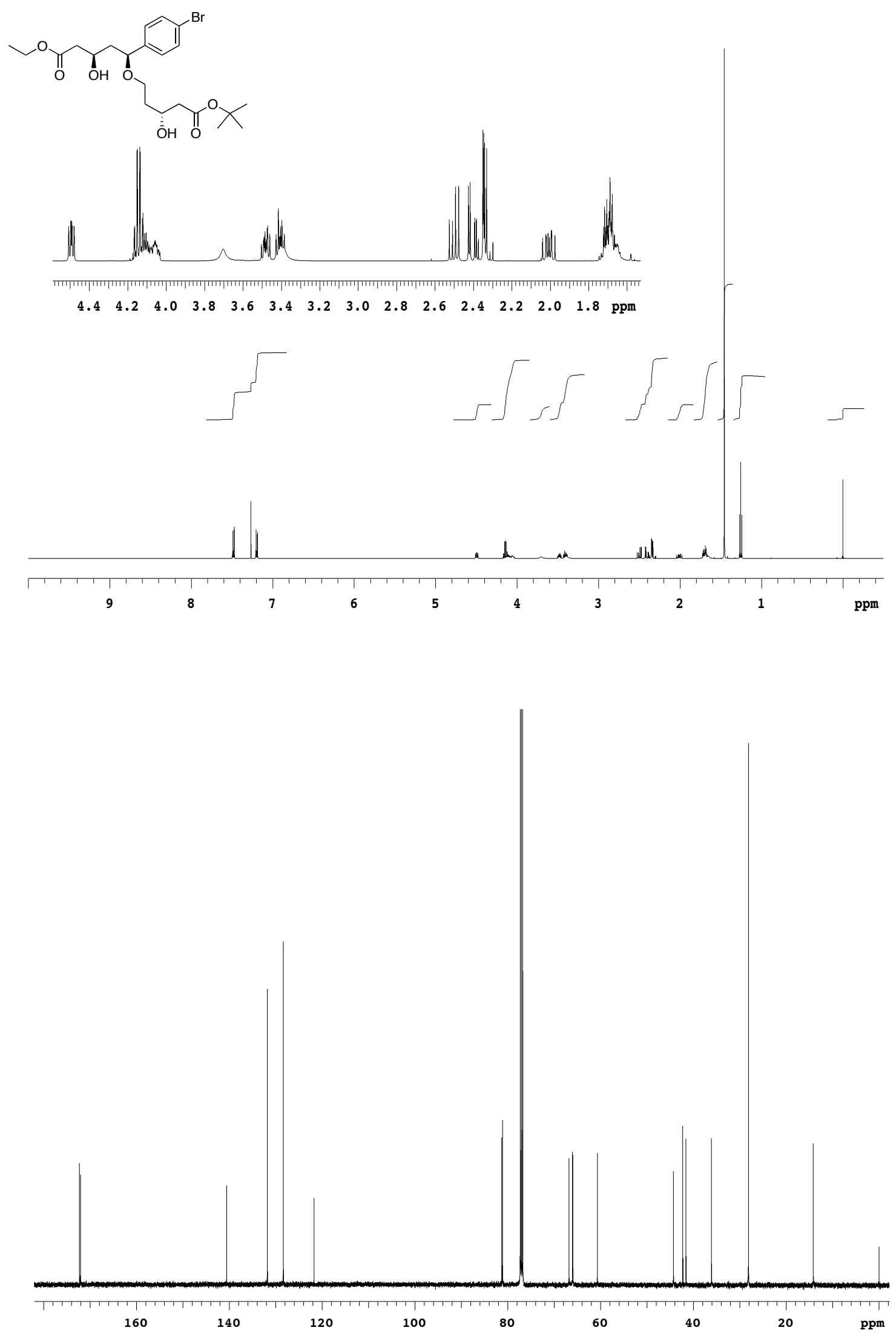

S-13 
Normal Phase HPLC analysis for 8: achiral column; isocratic 4\% $i$-PrOH / hexanes.

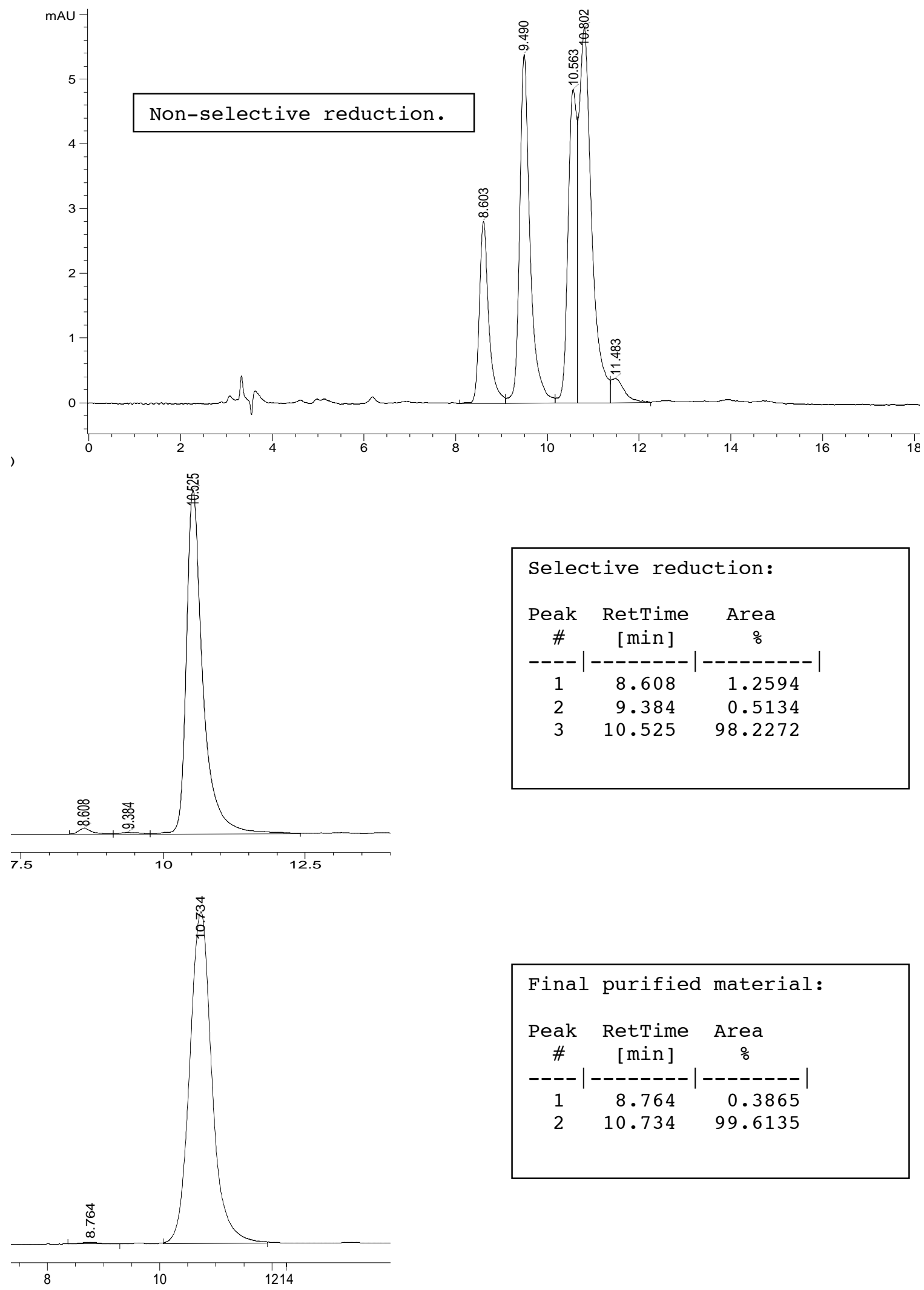




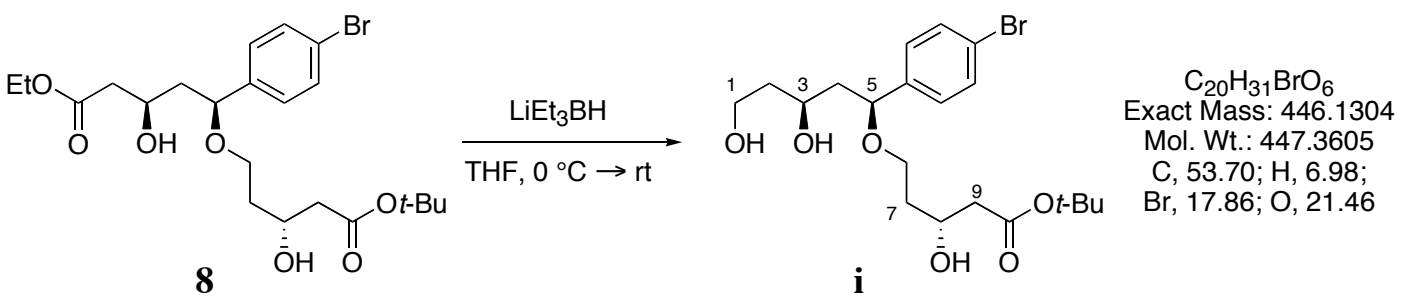

To a solution of diol $8(106 \mathrm{mg}, 0.217 \mathrm{mmol})$ in THF $(2.2 \mathrm{~mL})$, under $\mathrm{N}_{2}$ at $0{ }^{\circ} \mathrm{C}$, was added Super Hydride $(910 \mu \mathrm{L}, 1.0 \mathrm{M}$ solution of lithium triethylborohydride in THF, $0.910 \mathrm{mmol})$ dropwise over $1 \mathrm{~min}$ via syringe. The reaction was stirred for $1.25 \mathrm{~h}$ at $0{ }^{\circ} \mathrm{C}$ then the bath was removed and the reaction was allowed to stir at $\mathrm{rt}$ for $30 \mathrm{~min}$. The reaction was poured into saturated aq. $\mathrm{NH}_{4} \mathrm{Cl}(25 \mathrm{~mL})$ and extracted with EtOAc $(3 \times 25 \mathrm{~mL})$. The combined organic layers were concentrated and redissolved in THF $(2 \mathrm{~mL})$. To this solution was added $\mathrm{H}_{2} \mathrm{O}_{2}(0.5 \mathrm{~mL}$, $30 \%$ ) followed by aq. $\mathrm{NaOH}(3$ drops, $3 \mathrm{~N}$ ) and the mixture was allowed to stir for $25 \mathrm{~min}$. Saturated aq. $\mathrm{NH}_{4} \mathrm{Cl}(2 \mathrm{~mL})$ was added and the layers were separated. The aqueous layer was extracted EtOAc $(3 \times 2 \mathrm{~mL})$. The combined organic layers were concentrated in vacuo and purified by preparative reverse phase HPLC $\left(5 \rightarrow 95 \% \mathrm{MeCN} / \mathrm{H}_{2} \mathrm{O}\right)$ to give $68.0 \mathrm{mg}$ of triol $\mathbf{i}$ as a colorless oil (70\%) and $9.2 \mathrm{mg}$ of the recovered starting material (77\% at $91 \%$ conversion).

Data for i:

$\mathbf{R}_{f}=0.29(100 \%$ EtOAc $)-$ one black spot w/ p-anisaldehyde stain.

IR (thin film): 3404 (br), 2976, 1939, 2878, 1724, 1591, 1485, 1408, 1392, 1367, 1341, 1295, $1257,1154,1095,1070,1010,954,826 \mathrm{~cm}^{-1}$.

${ }^{1}$ HNMR $\left(500 \mathrm{MHz}, \mathrm{CDCl}_{3}\right) ; \delta 7.48(2 \mathrm{H}, \mathrm{m}, \operatorname{ArH}), 7.19(2 \mathrm{H}, \mathrm{m}, \operatorname{ArH}), 4.48(1 \mathrm{H}, \mathrm{dd}, J=3.8,10.0$ $\mathrm{Hz}, \mathrm{C} 5), 4.11(2 \mathrm{H}, \mathrm{m}, \mathrm{C} 3 \& \mathrm{C} 8), 4.00(1 \mathrm{H}, \mathrm{d}, J=1.2 \mathrm{~Hz},-\mathrm{OH}), 3.85(1 \mathrm{H}, \mathrm{tt}, J=4.8,6.0 \mathrm{~Hz}, \mathrm{C} 1)$, $3.80(1 \mathrm{H}, \mathrm{tt}, J=4.5,6.4 \mathrm{~Hz}, \mathrm{C} 1), 3.49(1 \mathrm{H}, \mathrm{dd}, J=6.3,6.8,9.6 \mathrm{~Hz}, \mathrm{C} 6), 3.43(1 \mathrm{H}, \mathrm{d}, J=3.9 \mathrm{~Hz}$, -OH), $3.42(1 \mathrm{H}, \mathrm{dt}, J=5.8,9.6 \mathrm{~Hz}, \mathrm{C} 6), 2.83(1 \mathrm{H}, \mathrm{dd}, J=4.3,6.3, \mathrm{C} 1-\mathrm{OH}), 2.37(1 \mathrm{H}, \mathrm{dd}, J=$ 4.2, 16.4, C9), $2.33(1 \mathrm{H}, \mathrm{dd}, J=8.1,16.4, \mathrm{C} 9), 1.99(1 \mathrm{H}, \mathrm{dt}, J=14.6,9.7 \mathrm{~Hz}, \mathrm{C} 4), 1.69(5 \mathrm{H}, \mathrm{m}$, $\mathrm{C} 4, \mathrm{C} 7$ \& $\mathrm{C} 2), 1.46$ (9H, s, t-Bu).

${ }^{13}$ CNMR (125 MHz, $\mathrm{CDCl}_{3}$ ): $\delta$ 172.1, 140.7, 131.8 (2C), 128.1 (2C), 121.7, 82.6, 81.4, 71.4, $65.8,65.6,61.2,43.3,42.2,38.6,36.0,28.1(3 \mathrm{C})$.

HRMS (FAB+): Calculated for $\mathrm{C}_{20} \mathrm{H}_{31} \mathrm{BrO}_{6} \mathrm{Na}[\mathrm{M}+\mathrm{Na}]$ : 469.1202. Found: 469.1184.

Elemental Analysis: Calculated for $\mathrm{C}_{20} \mathrm{H}_{31} \mathrm{BrO}_{6}$ : C 53.70; H 6.98. Found: C 53.89; H 7.11.

$[\alpha]_{D}^{25}=-27.8^{\circ}\left(c=0.88, \mathrm{C}_{6} \mathrm{H}_{6}\right)$. 
<smiles>CC(C)(C)OC(=O)CC[C@H](O)CCOC(CC(O)CCO)c1ccc(Br)cc1</smiles>

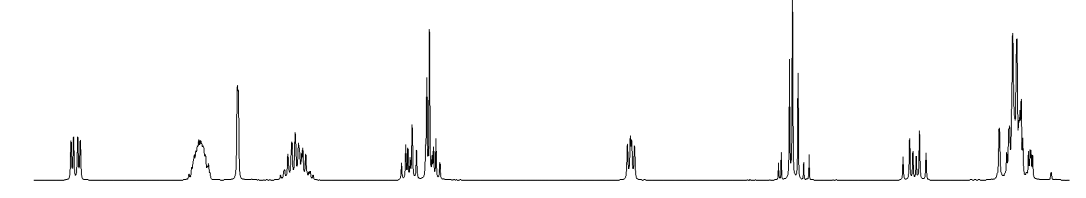

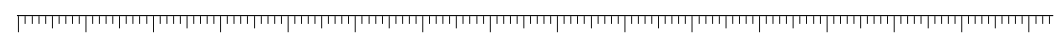
$\begin{array}{lllllllllllllllll}4.6 & 4.4 & 4.2 & 4.0 & 3.8 & 3.6 & 3.4 & 3.2 & 3.0 & 2.8 & 2.6 & 2.4 & 2.2 & 2.0 & 1.8 & \mathrm{ppm}\end{array}$
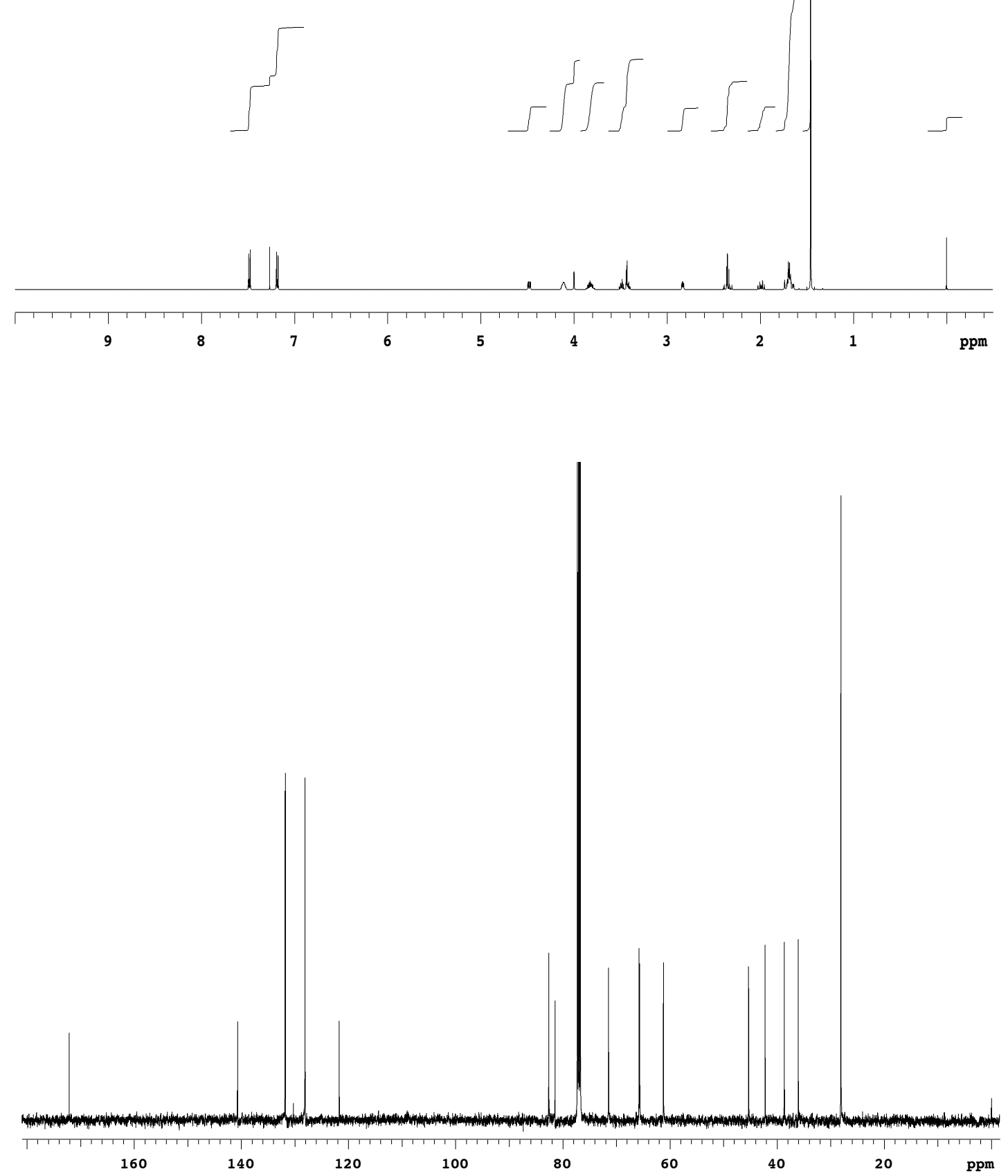


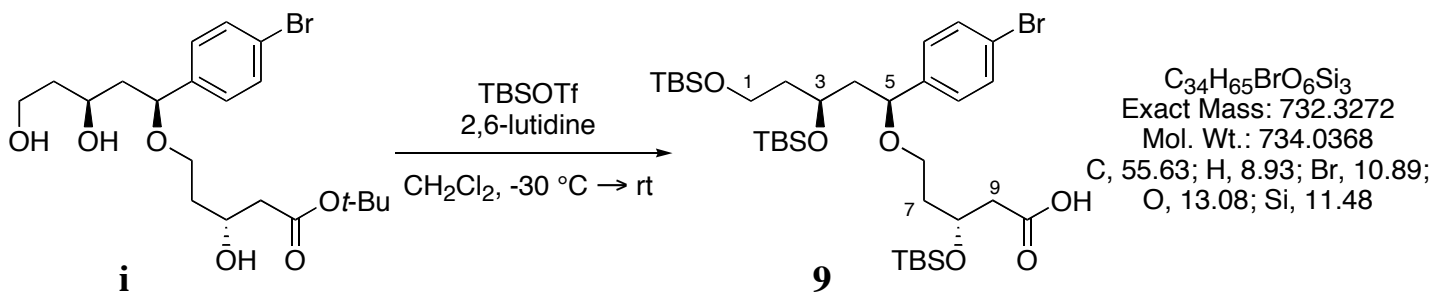

To a solution of triol $\mathbf{i}(42.3 \mathrm{mg}, 0.0945 \mathrm{mmol})$ in $\mathrm{CH}_{2} \mathrm{Cl}_{2}(190 \mu \mathrm{L})$, under $\mathrm{N}_{2}$ at rt, was added 2,6-lutidine $(331 \mu \mathrm{L}, 2.84 \mathrm{mmol})$ in one portion via syringe. The flask was placed in a $-30{ }^{\circ} \mathrm{C}$ bath and TBSOTf $(217 \mu \mathrm{L}, 0.945 \mathrm{mmol})$ was added in one portion via syringe. The bath was allowed to warm to $0{ }^{\circ} \mathrm{C}$ over $40 \mathrm{~min}$ and then the reaction was removed from the bath and allowed to stir at $\mathrm{rt}$ for $23 \mathrm{hr}$. $\mathrm{NaOH}\left(2 \mathrm{~mL}, 1 \mathrm{~N}\right.$ in 3:1:1 MeOH:THF: $\left.\mathrm{H}_{2} \mathrm{O}\right)$ was added to the reaction and the color of the solution was observed to change from pink to a light yellow color. The biphasic mixture was stirred for $1.5 \mathrm{~h}$ and then solid $\mathrm{NaHSO}_{4}$ was added until the $\mathrm{pH}$ of the aqueous phase reached 3 . The mixture was extracted with $\mathrm{Et}_{2} \mathrm{O}(3 \times 4 \mathrm{~mL})$ and the combined organic layers were dried over anhydrous $\mathrm{MgSO}_{4}$, filtered and concentrated in vacuo. Chromatography on silica (10\% EtOAc / pentane $+1 \% \mathrm{AcOH})$ gave $56.2 \mathrm{mg}$ of carboxylic acid 9 as a colorless oil $(81 \%)$.

Data for 9:

$\mathbf{R}_{f}=0.57$ (20\% EtOAc / pentane) - one black spot w/ p-anisaldehyde stain.

IR (thin film): 2960 (br), 2955, 2929, 2885, 2857, 1713, 1472, 1394, 1361, 1293, 1256, 1187, 1096, 1010, 939, 836, $775 \mathrm{~cm}^{-1}$.

${ }^{1}$ HNMR $\left(500 \mathrm{MHz}, \mathrm{CDCl}_{3}\right): \delta 7.45(2 \mathrm{H}, \mathrm{m}, \mathrm{ArH}), 7.14(2 \mathrm{H}, \mathrm{m}, \mathrm{ArH}), 4.28(1 \mathrm{H}, \mathrm{dd}, J=5.2,8.0$ $\mathrm{Hz}, \mathrm{C} 5), 4.25(1 \mathrm{H}$, quint, $J=5.9 \mathrm{~Hz}, \mathrm{C} 8), 3.87(1 \mathrm{H}, \mathrm{tt}, J=5.2,6.6 \mathrm{~Hz}, \mathrm{C} 3), 3.66(1 \mathrm{H}, \mathrm{dt}, J=$ $10.3,6.5 \mathrm{~Hz}, \mathrm{C} 1), 3.64(1 \mathrm{H}, \mathrm{dt}, J=9.4,5.7 \mathrm{~Hz}, \mathrm{C} 1), 3.27(1 \mathrm{H}, \mathrm{dt}, J=9.4,6.0 \mathrm{~Hz}, \mathrm{C} 6), 3.24(1 \mathrm{H}$, ddd, $J=5.6,7.2,9.3 \mathrm{~Hz}, \mathrm{C} 6), 2.47(1 \mathrm{H}, \mathrm{dd}, J=5.3,15.1 \mathrm{~Hz}, \mathrm{C} 9), 2.40(1 \mathrm{H}, \mathrm{dd}, J=6.0,15.1$, C9), $1.96(1 \mathrm{H}, \mathrm{ddd}, J=5.3,8.1,13.9 \mathrm{~Hz}, \mathrm{C} 4), 1.71-1.79(5 \mathrm{H}, \mathrm{m}, \mathrm{C} 4, \mathrm{C} 7 \& \mathrm{C} 2), 0.89(9 \mathrm{H}, \mathrm{s},-$ OTBDMS), 0.86 (9H, s, -OTBDMS), 0.84 (9H, s, -OTBDMS), 0.07 (3H, s, -OTBDMS), 0.06 (3H, s, -OTBDMS), 0.04 (3H, s, -OTBDMS), 0.03 (3H, s, -OTBDMS), 0.017 (3H, s, OTBDMS), 0.016 (3H, s, -OTBDMS).

${ }^{13}$ CNMR (100 MHz, $\mathrm{CDCl}_{3}$ ): $\delta$ 175.0, 141.7, 131.6 (2C), 128.4 (2C), 121.4, 79.0, 66.9, 66.5, 64.7, 59.8, 45.8, 41.8, 40.0, 37.2, 25.9 (x6), 25.7 (3C), 18.2, 18.0, 17.9, -4.37, -4.42, -4.67, -4.88, $-5.35(2 \mathrm{C})$.

HRMS (MALDI): Calculated for $\mathrm{C}_{34} \mathrm{H}_{65} \mathrm{BrO}_{6} \mathrm{NaSi}_{3}$ [M+Na]: 755.3165. Found: 755.3154.

Elemental Analysis: Calculated for $\mathrm{C}_{34} \mathrm{H}_{65} \mathrm{BrO}_{6} \mathrm{Si}_{3}$ : C 55.63; H 8.93. Found: C 55.81; $\mathrm{H} 9.05$.

$[\alpha]_{D}^{26}=-15.9^{\circ}\left(c=0.64, \mathrm{CDCl}_{3}\right)$. 

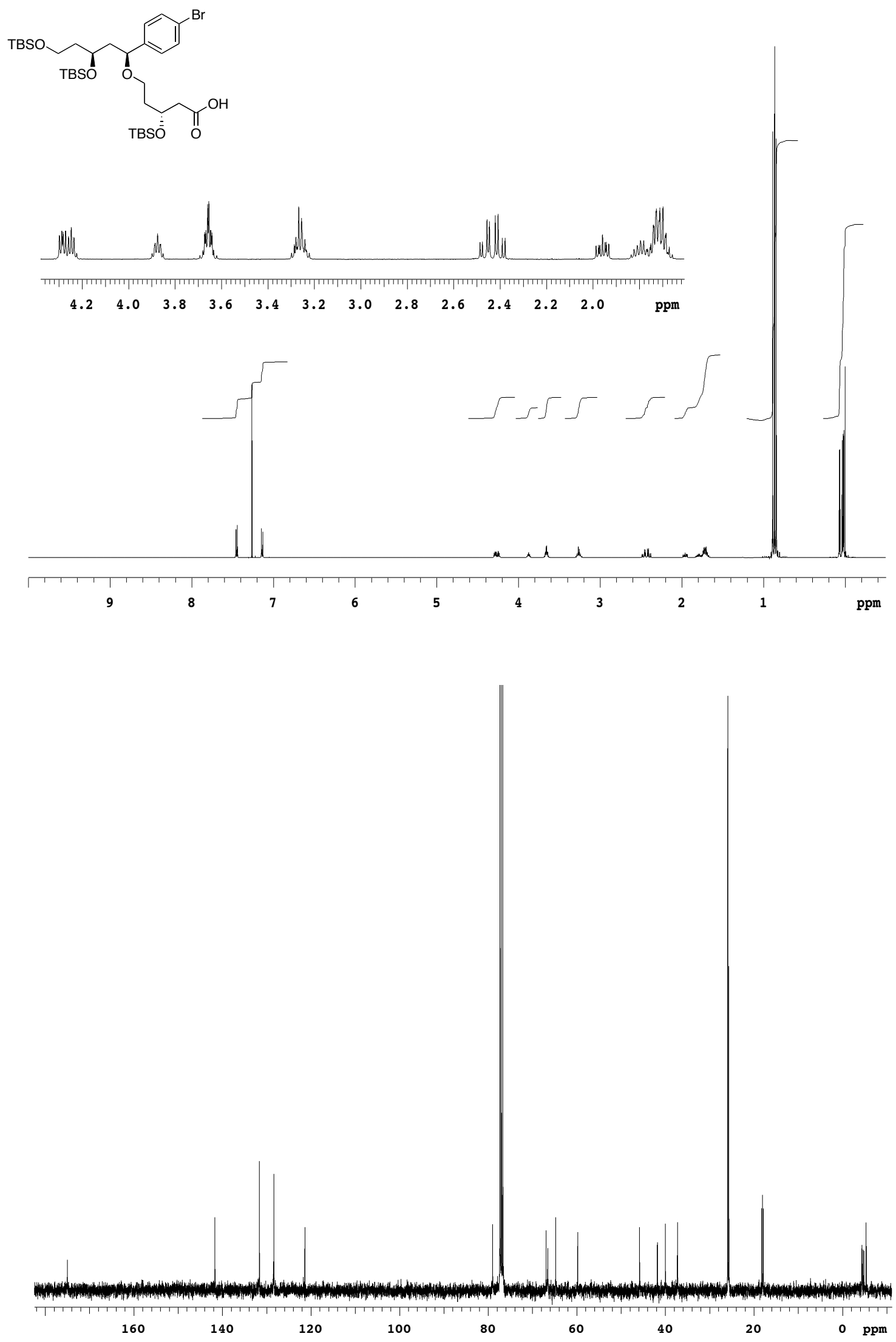

S-18 


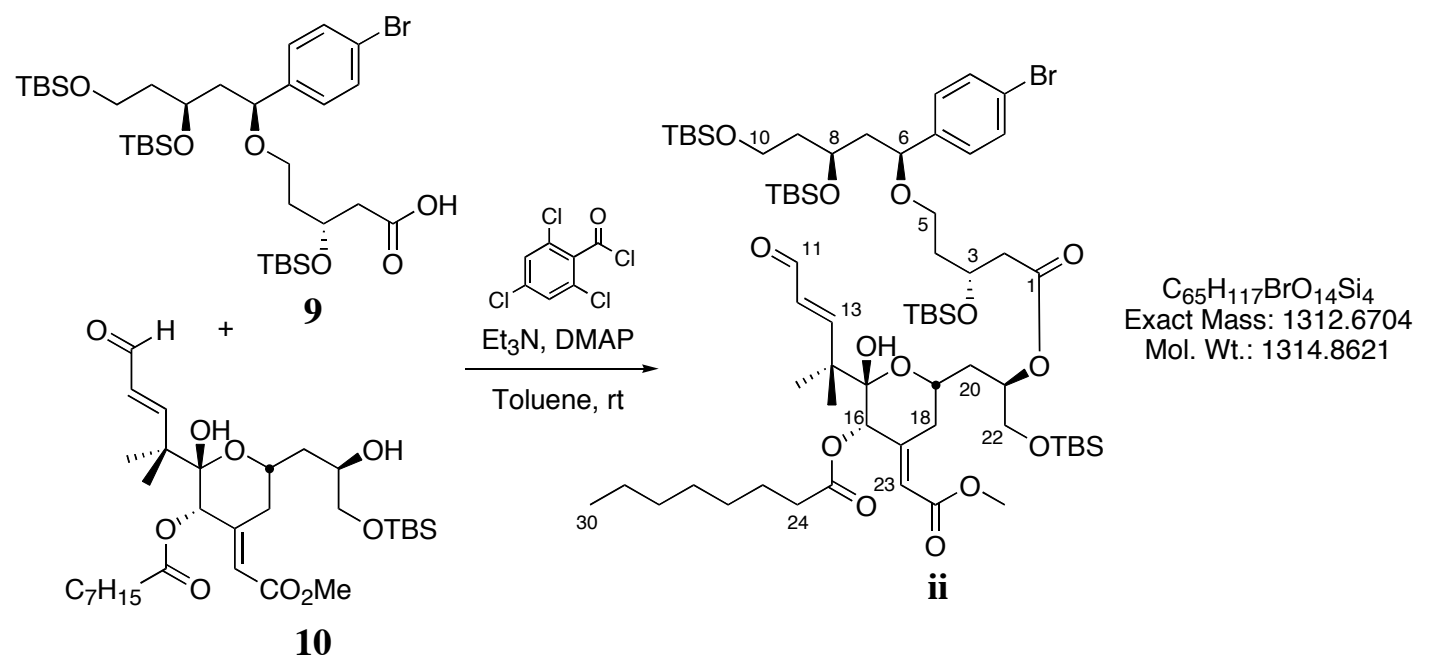

To a solution of carboxylic acid $9(33.7 \mathrm{mg}, 45.9 \mu \mathrm{mol})$ in $\mathrm{MePh}(2.1 \mathrm{~mL})$ at $\mathrm{rt}$ under $\mathrm{N}_{2}$, was added a solution of 2,4,6-trichlorobenzoyl chloride in toluene $(0.46 \mathrm{M}, 100 \mu \mathrm{L}, 46 \mathrm{mmol})$ in one portion via syringe. To this solution was added $\mathrm{Et}_{3} \mathrm{~N}(24.4 \mu \mathrm{L}, 0.175 \mathrm{mmol})$ in one portion via syringe and the solution was allowed to stir for $4 \mathrm{~h}$. To this mixture was added a solution of alcohol $10(25.0 \mathrm{mg}, 41.7 \mu \mathrm{mol})$ and DMAP $(25.5 \mathrm{mg}, 0.209 \mathrm{mmol})$ in $\mathrm{MePh}(2.5 \mathrm{~mL})$ in one portion via syringe. The cloudy mixture was stirred for $1.75 \mathrm{~h}$, the solvent volume was reduced by $50 \%$ in vacuo and the remaining mixture was directly chromatographed on silica (10\% EtOAc / pentane) to give $44.5 \mathrm{mg}$ of the product ii as a colorless oil (81\%).

Data for ii:

$\mathbf{R}_{f}=0.69$ (15\% EtOAc, 85\% pentane) - one black spot $\mathrm{w} / \mathrm{p}$-anisaldehyde stain.

IR (thin film): 3483 (w), 2945, 2929, 2857, 1725, 1692, 1471, 1464, 1436, 1386, 1361, 1300, $1256,1228,1156,1096,1036,1010,983,939,836,776 \mathrm{~cm}^{-1}$.

${ }^{1}$ HNMR $\left(500 \mathrm{MHz}, \mathrm{CDCl}_{3}\right): \delta 9.57(1 \mathrm{H}, \mathrm{d}, J=7.8 \mathrm{~Hz}, \mathrm{C} 11), 7.44(2 \mathrm{H}, \mathrm{m}, \mathrm{Ar}-\mathbf{H}), 7.41(1 \mathrm{H}, \mathrm{d}, J$ $=16.0 \mathrm{~Hz}, \mathrm{C} 13), 7.14(2 \mathrm{H}, \mathrm{m}, \mathrm{Ar}-\mathbf{H}), 6.03(1 \mathrm{H}, \mathrm{d}, J=2.0 \mathrm{~Hz}, \mathrm{C} 23), 5.96(1 \mathrm{H}, \mathrm{dd}, J=7.8,16.1$ $\mathrm{Hz}, \mathrm{C} 12), 5.20(1 \mathrm{H}$, ddt $J=1.2,10.7,5.0 \mathrm{~Hz}, \mathrm{C} 21), 5.13(1 \mathrm{H}, \mathrm{s}, \mathrm{C} 16), 4.28(1 \mathrm{H}, \mathrm{dd}, J=5.5,7.6$ $\mathrm{Hz}, \mathrm{C} 6), 4.22(1 \mathrm{H}, \mathrm{m}, \mathrm{C} 3), 3.85(1 \mathrm{H}$, quint, $J=6.0 \mathrm{~Hz}, \mathrm{C} 8), 4.08(1 \mathrm{H}, \mathrm{tt}, J=1.8,11.3 \mathrm{~Hz}, \mathrm{C} 19)$, $3.69(3 \mathrm{H}, \mathrm{s},-\mathrm{OMe}), 3.61-3.71(5 \mathrm{H}, \mathrm{m}, \mathrm{C} 10, \mathrm{C} 22, \& \mathrm{C} 18), 3.25(1 \mathrm{H}, \mathrm{dt}, J=9.2,6.4 \mathrm{~Hz}, \mathrm{C} 5), 3.21$ $(1 \mathrm{H}, \mathrm{dt}, J=9.2,6.7 \mathrm{~Hz}, \mathrm{C} 5), 3.11(1 \mathrm{H}, \mathrm{s}, \mathrm{C} 15-\mathrm{OH}), 2.41(1 \mathrm{H}, \mathrm{dd}, J=6.9,15.1 \mathrm{~Hz}, \mathrm{C} 2), 2.36(1 \mathrm{H}$, $\mathrm{dd}, J=5.1,15.1 \mathrm{~Hz}, \mathrm{C} 2), 2.10(1 \mathrm{H}, \mathrm{dt}, J=16.3,7.4 \mathrm{~Hz}, \mathrm{C} 24), 2.07(1 \mathrm{H}, \mathrm{m}, \mathrm{C} 18), 2.02(1 \mathrm{H}, \mathrm{dt}, J$ $=16.3,7.6 \mathrm{~Hz}, \mathrm{C} 24), 1.94(2 \mathrm{H}, \mathrm{m}, \mathrm{C} 7 \& \mathrm{C} 20), 1.78(1 \mathrm{H}, \mathrm{ddd}, J=1.7,10.9,14.4 \mathrm{~Hz}, \mathrm{C} 20), 1.71$ $(5 \mathrm{H}, \mathrm{m}, \mathrm{C} 4 \&$ C7 \& C9), $1.49(1 \mathrm{H}, \mathrm{dt}, J=14.4,7.5 \mathrm{~Hz}, \mathrm{C} 25), 1.24(9 \mathrm{H}, \mathrm{m}, \mathrm{C} 25-\mathrm{C} 29), 1.22(3 \mathrm{H}$, $\left.\mathrm{s},-\mathbf{C H}_{3}\right), 1.16$ (3H, s, -CH $), 0.90$ (9H, s, -OTBDMS), 0.89 (9H, s, -OT BDMS), 0.88 (3H, m, C30), 0.86 (9H, s, -OTBDMS), 0.80 (9H, s, -OTBDMS), 0.071 (3H, s, -OTBDMS), 0.067 (3H, s, -OTBDMS), 0.038 (3H, s, -OTBDMS), 0.019 (3H, s, -OTBDMS), 0.016 (3H, s, -OTBDMS), 0.014 (3H, s, -OTBDMS), 0.005 (3H, s, -OTBDMS), -0.003 (3H, s, -OTBDMS).

${ }^{13}$ CNMR $\left(125 \mathrm{MHz}, \mathrm{CDCl}_{3}\right): \delta$ 194.6, 172.6, 171.7, 166.5, 166.4, 150.3, 141.8, 131.6 (2C), 128.4 (2C), 127.5, 121.3, 120.8, 99.5, 78.9, 72.5, 71.5, 66.53, 66.50, 66.1, 64.9, 64.8, 59.7, 51.2, 45.71, $45.72,42.4,40.1,37.5,37.3,34.5,31.6,30.8,28.89,28.86,25.91$ (3C), 25.88 (3C), 25.82 (3C), 25.7 (3C), 24.5, 23.0, 22.5, 20.0, 18.3, 18.2, 18.1, 17.9, 14.1, -4.3 (2C), -4.73, -4.74, -5.30, -5.32, $-5.34,-5.36$.

HRMS (MALDI): Calculated for $\mathrm{C}_{65} \mathrm{H}_{117} \mathrm{BrO}_{14} \mathrm{NaSi}_{4}$ [M+Na]: 1335.6596. Found: 1335.6634. 
$[\alpha]_{D}^{23}=-37.2^{\circ}\left(c=0.93, \mathrm{CH}_{2} \mathrm{Cl}_{2}\right)$. 

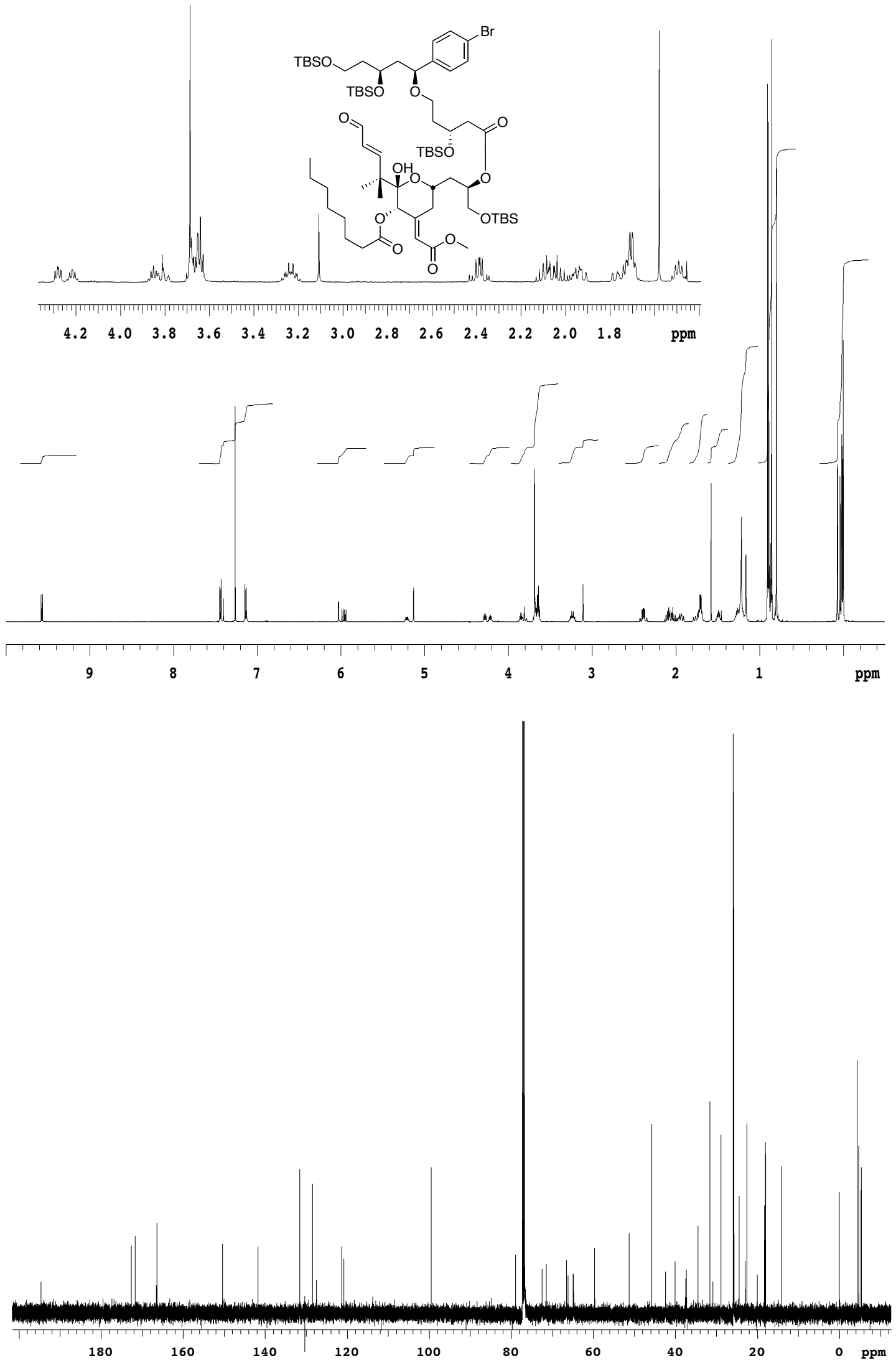


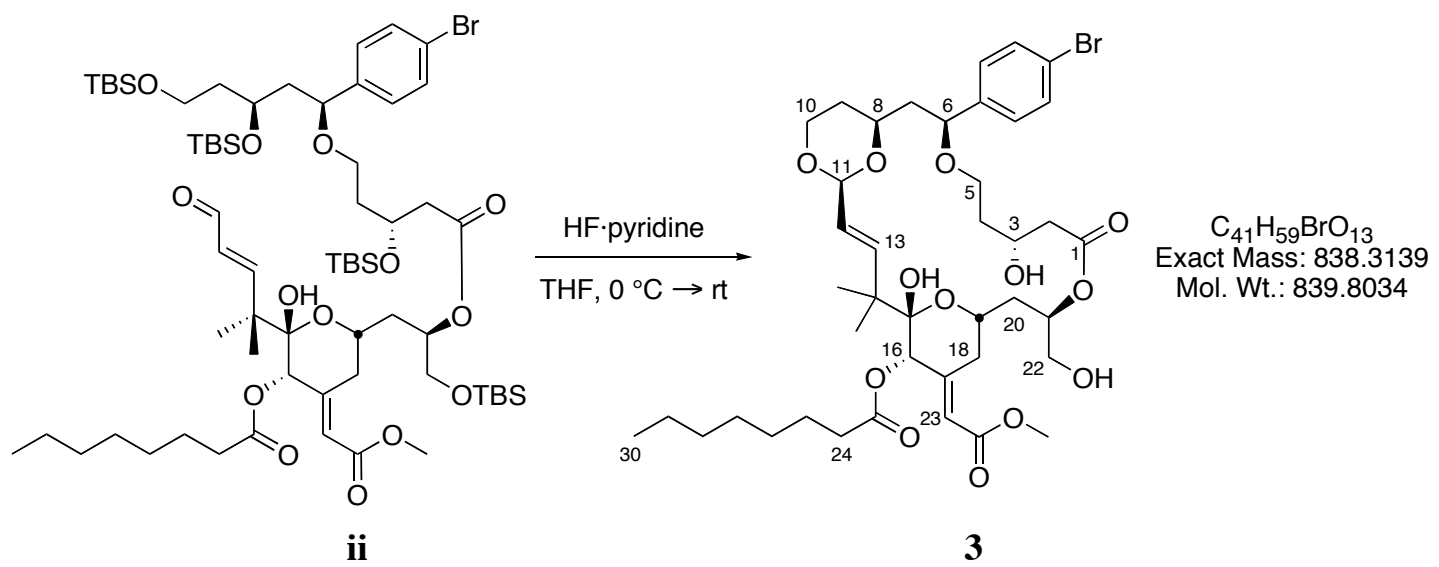

To a solution of the seco aldehyde ii $(44.5 \mathrm{mg}, 33.8 \mu \mathrm{mol})$ in THF $(9.0 \mathrm{~mL})$, under $\mathrm{N}_{2}$ at $0{ }^{\circ} \mathrm{C}$ in a polypropylene vial, was added HF.pyridine $(1.73 \mathrm{~mL})$ dropwise via syringe. The solution was stirred for $1 \mathrm{~h}$ at $0{ }^{\circ} \mathrm{C}$; the bath was removed and the reaction was allowed to stir for an additional $4.5 \mathrm{~h}$. The reaction solution was transferred to a separatory funnel containing water $(30 \mathrm{~mL})$ using EtOAc $(30 \mathrm{~mL})$. Saturated aq. $\mathrm{NaHCO}_{3}$ was added portionwise until gas evolution ceased (41 $\mathrm{mL}$, vigorous bubbling!). The layers were separated and the aqueous phase was extracted EtOAc $(3 \times 50 \mathrm{~mL})$. The combined organic layers were dried over anhydrous $\mathrm{Na}_{2} \mathrm{SO}_{4}$, filtered and concentrated in vacuo. Chromatography on silica $(60 \rightarrow 100 \%$ EtOAc / pentane) gave $23.7 \mathrm{mg}$ of analog 3 as a white amorphous solid (83\%).

Data for 3:

$\mathbf{R}_{f}=0.42(70 \%$ EtOAc, $30 \%$ pentane $)-$ one black spot $\mathrm{w} / \mathrm{p}$-anisaldehyde stain.

IR (thin film): 3461, 3334 (br), 2925, 2855, 1728, 1668, 1373, 1286, 1259, 1230, 1159, 1135, 1103, 1082, 1005, 980, $807 \mathrm{~cm}^{-1}$.

${ }^{1}$ HNMR $\left(600 \mathrm{MHz}, \mathrm{CDCl}_{3}\right): \delta 7.47(2 \mathrm{H}, \mathrm{m}, \mathrm{Ar}-\mathrm{H}), 7.14(2 \mathrm{H}, \mathrm{m}, \mathrm{Ar}-\mathbf{H}), 6.00(1 \mathrm{H}, \mathrm{d}, J=15.9 \mathrm{~Hz}$, C13), $6.00(1 \mathrm{H}, \mathrm{d}, J=2.0 \mathrm{~Hz}, \mathrm{C} 23), 5.43(1 \mathrm{H}, \mathrm{dd}, J=7.3,15.9 \mathrm{~Hz}, \mathrm{C} 12), 5.43(1 \mathrm{H}, \mathrm{m}, \mathrm{C} 21)$, $5.15(1 \mathrm{H}, \mathrm{s}, \mathrm{C} 16$ or $\mathrm{C} 15-\mathrm{OH}), 5.10(1 \mathrm{H}, \mathrm{s}, \mathrm{C} 16$ or $\mathrm{C} 15-\mathrm{OH}), 5.09(1 \mathrm{H}, \mathrm{d}, J=7.3 \mathrm{~Hz}, \mathrm{C} 11), 4.36$ $(1 \mathrm{H}, \mathrm{d}(\mathrm{br}), J=11.7 \mathrm{~Hz}, \mathrm{C} 3-\mathrm{OH}), 4.32(1 \mathrm{H}, \mathrm{dd}, J=2.4,11.7 \mathrm{~Hz}, \mathrm{C} 6), 4.28(1 \mathrm{H}, \mathrm{m}, \mathrm{C} 3), 4.06$ $(3 \mathrm{H}, \mathrm{m}, \mathrm{C} 8, \mathrm{C} 10$ \& $\mathrm{C} 19), 3.90(1 \mathrm{H}, \mathrm{dt}, J=2.2,12.2 \mathrm{~Hz}, \mathrm{C} 10), 3.86(1 \mathrm{H}, \mathrm{dd}, J=2.9,12.0 \mathrm{~Hz}$, C22), $3.72(1 \mathrm{H}, \mathrm{dd}, J=2.2,13.9 \mathrm{~Hz}, \mathrm{C} 18), 3.68(3 \mathrm{H}, \mathrm{s},-\mathrm{OMe}), 3.66(1 \mathrm{H}, \mathrm{dd}, J=5.9,12.0 \mathrm{~Hz}$, C22), $3.41(1 \mathrm{H}, \mathrm{ddd}, J=1.7,5.1,9.5 \mathrm{~Hz}, \mathrm{C} 5), 3.30(1 \mathrm{H}, \mathrm{ddd}, J=2.7,9.8,12.9 \mathrm{~Hz}, \mathrm{C} 5), 2.54(1 \mathrm{H}$, $\mathrm{dd}, J=10.7,12.9 \mathrm{~Hz}, \mathrm{C} 2), 2.51(1 \mathrm{H}, \mathrm{dd}, J=2.9,12.7 \mathrm{~Hz}, \mathrm{C} 2), 2.30(2 \mathrm{H}, \mathrm{m}, \mathrm{C} 24), 2.22(1 \mathrm{H}$, dddd, $J=2.7,5.1,12.9,15.6 \mathrm{~Hz}, \mathrm{C} 4), 2.04(3 \mathrm{H}, \mathrm{m}, \mathrm{C} 7, \mathrm{C} 20 \& \mathrm{C} 18), 1.81(1 \mathrm{H}, \mathrm{ddd}, J=2.6,11.7$, $14.2 \mathrm{~Hz}, \mathrm{C} 20), 1.75(1 \mathrm{H}, \mathrm{dq}, J=4.6,12.5 \mathrm{~Hz}, \mathrm{C} 9), 1.60(3 \mathrm{H}, \mathrm{m}, \mathrm{C} 7 \& \mathrm{C} 25), 1.49$ (1H, d (br), $J=$ $14.9 \mathrm{~Hz}, \mathrm{C} 4), 1.38(1 \mathrm{H}, \mathrm{d}(\mathrm{br}), J=13.0 \mathrm{~Hz}, \mathrm{C} 9), 1.26(8 \mathrm{H}, \mathrm{m}, \mathrm{C} 26-\mathrm{C} 29), 1.19\left(3 \mathrm{H}, \mathrm{s},-\mathrm{C}\left(\mathrm{CH}_{3}\right)_{2}\right)$, $1.04\left(3 \mathrm{H}, \mathrm{s},-\mathrm{C}\left(\mathrm{CH}_{3}\right)_{2}\right), 0.86(3 \mathrm{H}, \mathrm{t}, J=7.2 \mathrm{~Hz}, \mathrm{C} 30)$.

${ }^{13}$ CNMR (100 MHz, $\left.\mathrm{CDCl}_{3}\right): \delta 172.3,172.1,167.0,151.6,142.6,140.2,131.9$ (2C), 128.1 (2C), $125.9,121.9,119.9,102.3,99.0,83.4,75.7,74.1,71.6,68.7,66.2,65.9,65.7,64.6,51.1,45.5$, 45.1, 42.1, 35.9, 34.6, 33.6, 32.3, 31.6, 31.0, 29.0, 28.9, 24.7, 24.3, 22.6, 19.4, 14.0.

HRMS (FAB+): Calculated for $\mathrm{C}_{41} \mathrm{H}_{59} \mathrm{BrO}_{13} \mathrm{Na}[\mathrm{M}+\mathrm{Na}]$ : 861.3031. Found: 861.2991.

$[\alpha]_{D}^{27}=-122.3^{\circ}\left(c=0.32, \mathrm{C}_{6} \mathrm{H}_{6}\right)$. 

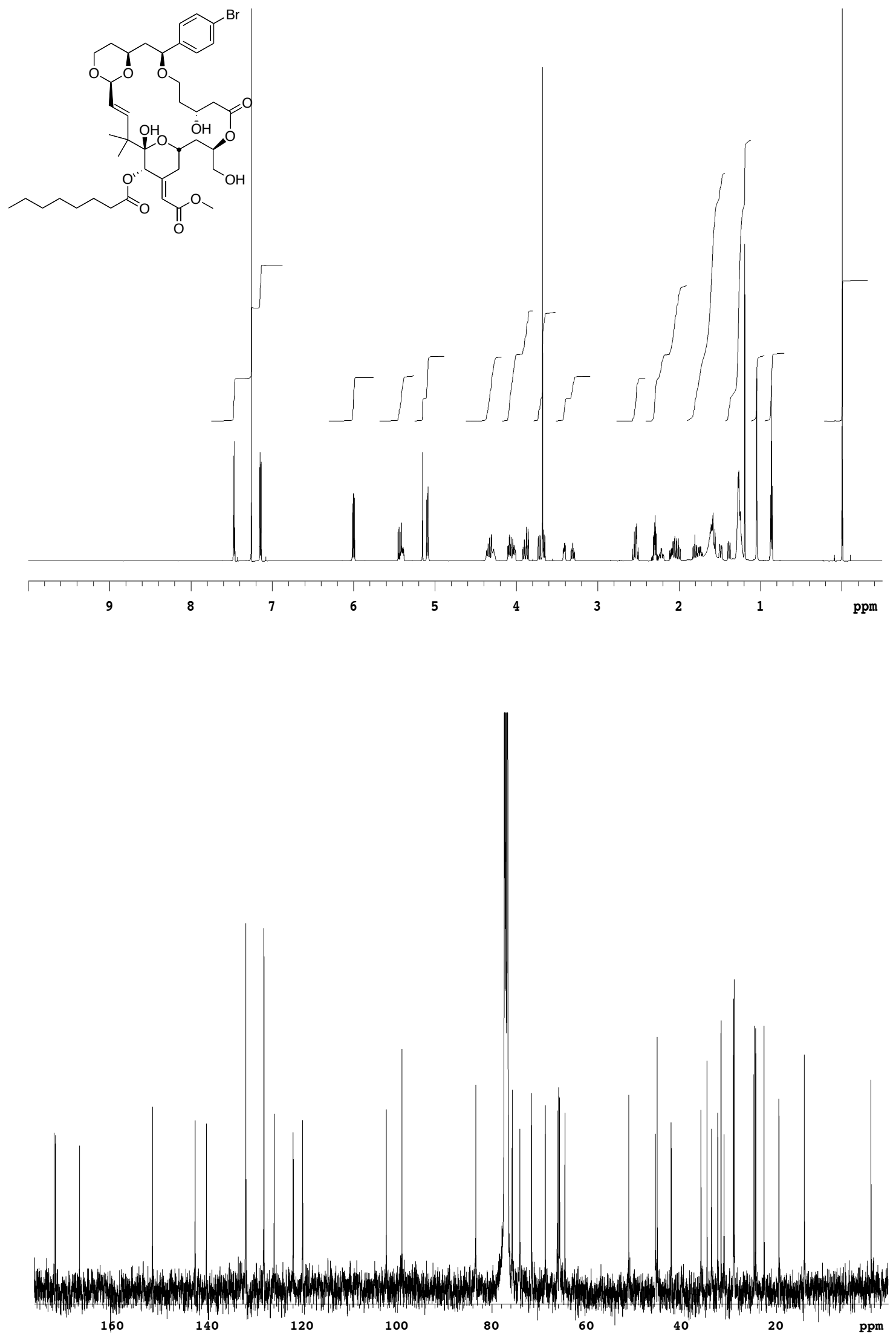

S-23 


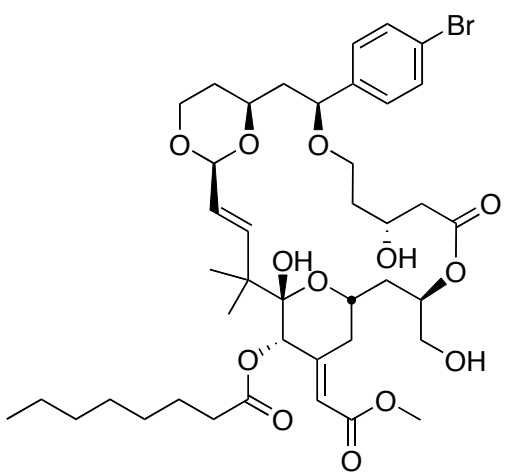

3

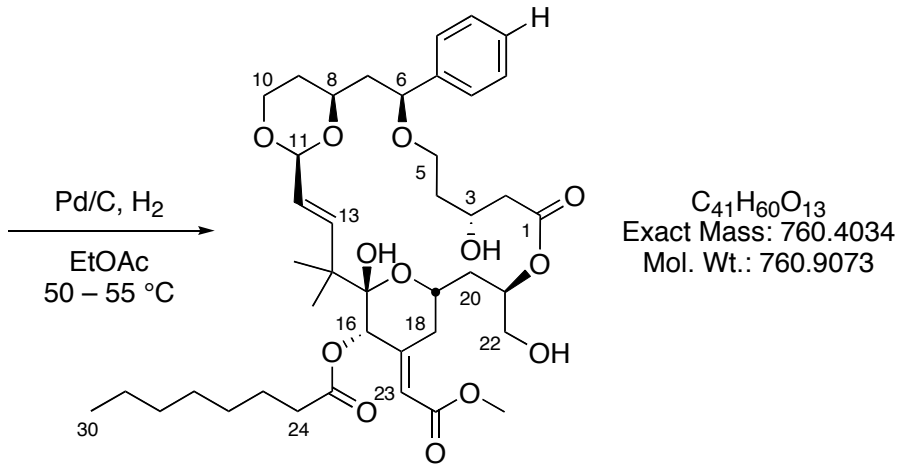

2

To a solution of analog $3(1.2 \mathrm{mg}, 1.4 \mu \mathrm{mol})$ in EtOAc $(0.2 \mathrm{~mL})$, was added $\mathrm{Pd} / \mathrm{C}(10 \% \mathrm{wt} \mathrm{Pd}$, $1.0 \mathrm{mg}$ ). The vial was purged with $\mathrm{H}_{2}$ and the reaction was allowed to stir under 1 atm of $\mathrm{H}_{2}$ in an oil bath maintained at $50-55^{\circ} \mathrm{C}$ for $4 \mathrm{~h}$. The reaction mixture was filtered through a plug of celite using EtOAc. The concentrated filtrate was purified by reverse phase HPLC $(65 \rightarrow 90 \%$ $\mathrm{MeCN} / \mathrm{H}_{2} \mathrm{O}$ ) to give $0.8 \mathrm{mg}$ of analog 2 as a white amorphous solid (73\%).

Data for 2:

RP-HPLC: Co-injection of $\mathbf{2}$ with an equimolar amount of a sample of $\mathbf{2}$ prepared by the old synthetic route ${ }^{4}$ showed only one peak.

${ }^{1}$ HNMR: Proton NMR of 2 matched the spectrum obtained for 2 prepared by the old synthetic route.

LRMS (ESI+) Calculated for $\mathrm{C}_{41} \mathrm{H}_{60} \mathrm{O}_{13} \mathrm{Na}[\mathrm{M}+\mathrm{Na}]$ : 783.4. Found: 783.6.

${ }^{4}$ Wender, P. A.; Clarke, M. O.; Horan, J. C. Org. Lett. 2005, 7, 1995-1998. 


\section{HNMR of 2:}

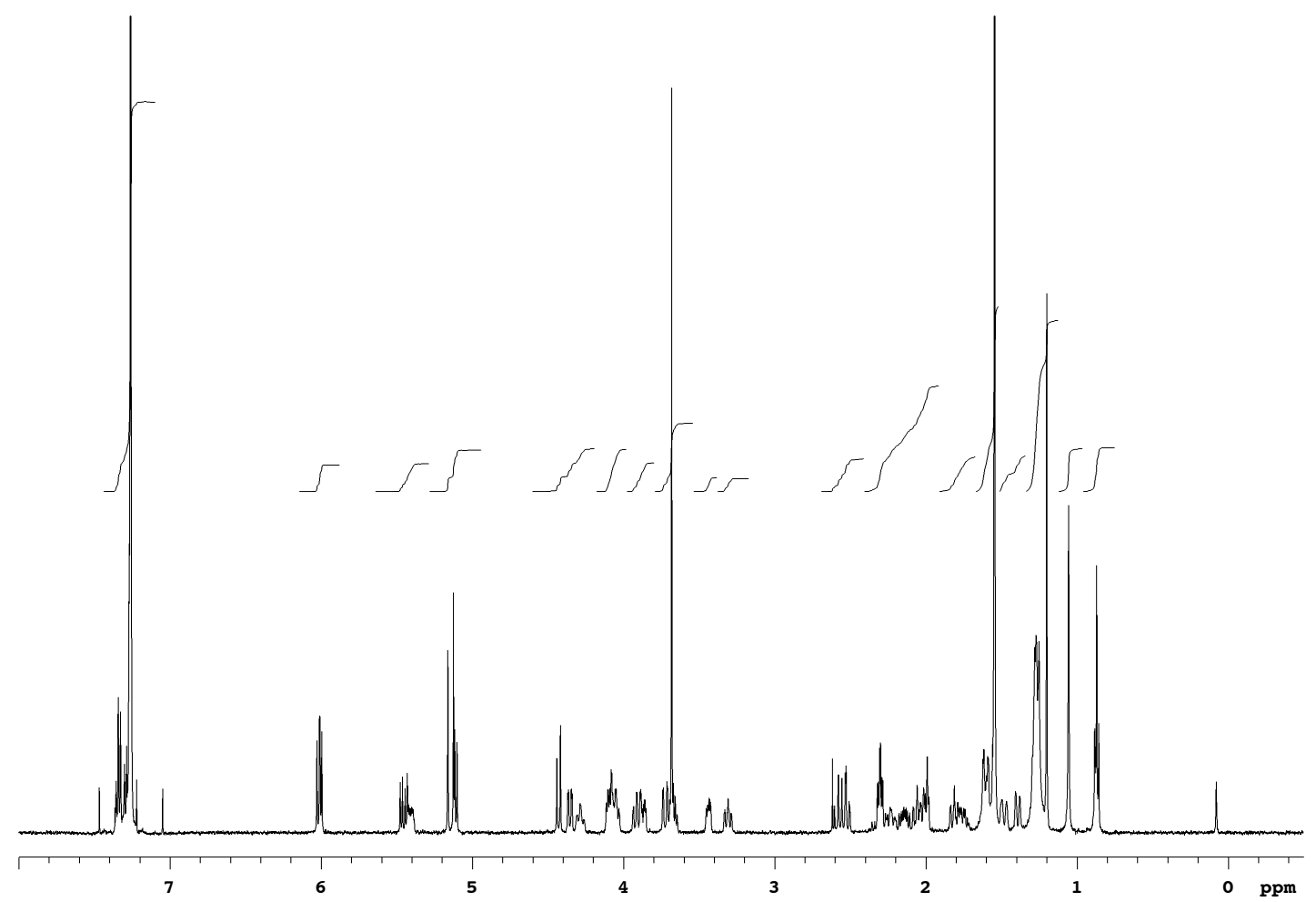

HNMR of $\mathbf{2}$ prepared by the old synthetic route:

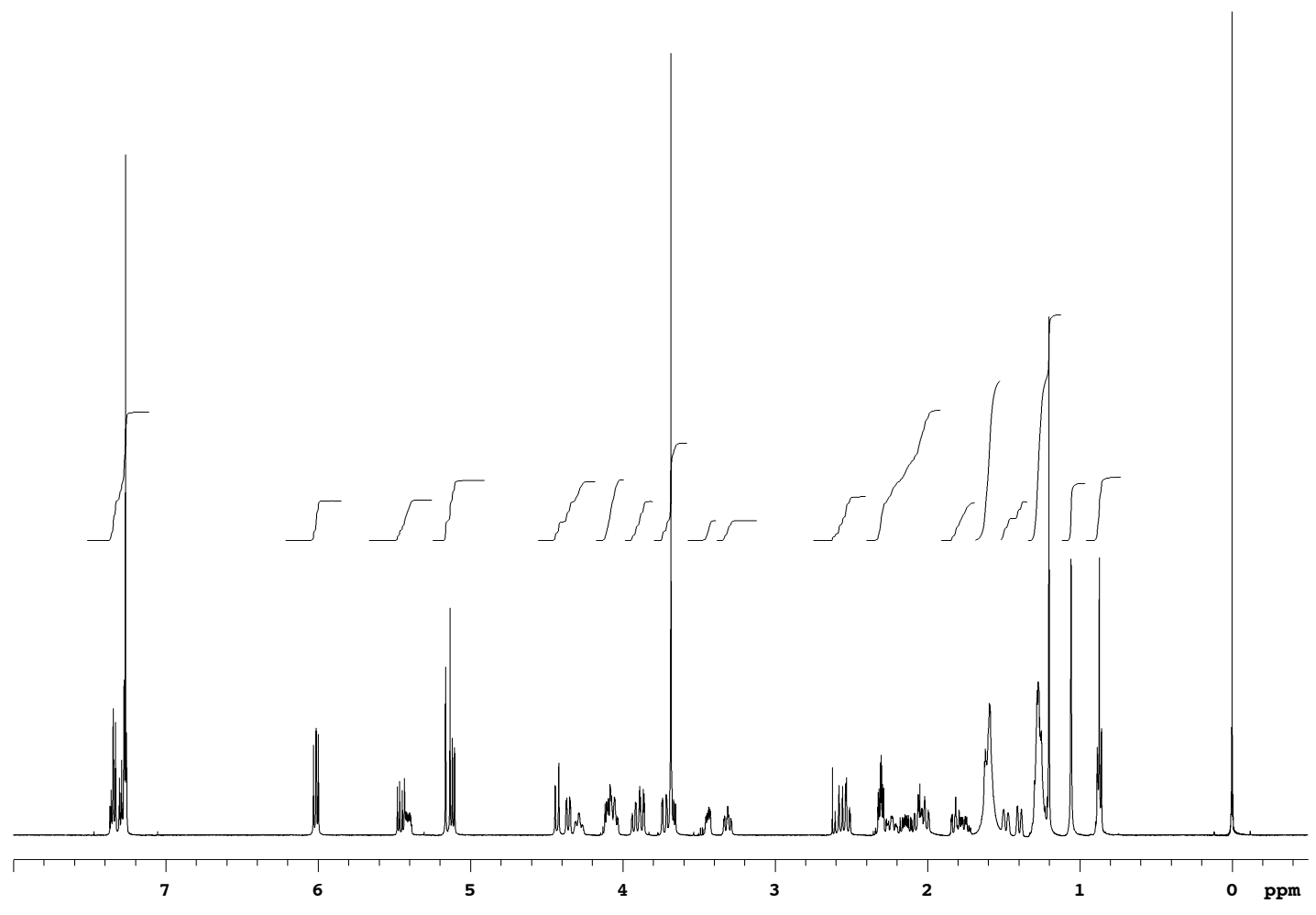




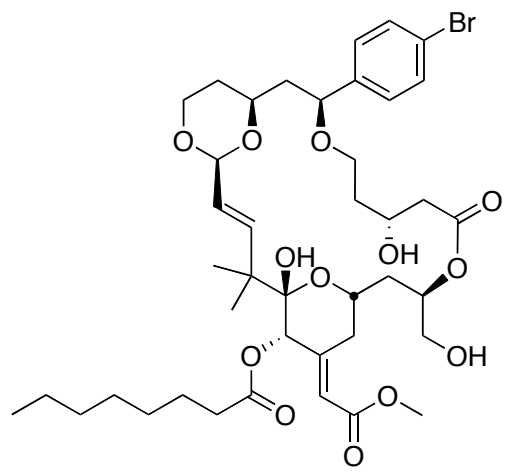

3

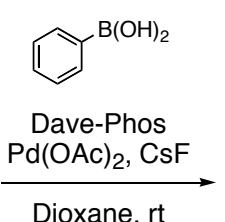

Dioxane, rt

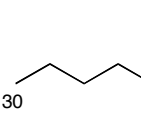

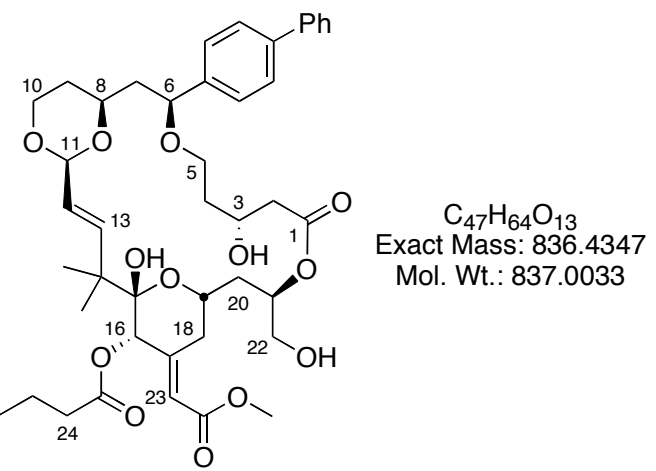

11

Aryl bromide $3(3.0 \mathrm{mg}, 3.6 \mu \mathrm{mol})$ and $\mathrm{CsF}(4.0 \mathrm{mg}, 26 \mu \mathrm{mol})$ were measured into a dry 2 $\mathrm{mL}$ glass vial and placed under high vacuum for $10 \mathrm{~min}$. The vial was removed from the vacuum under $\mathrm{N}_{2}$ gas and placed under an $\mathrm{N}_{2}$ atmosphere. To this vial was added phenylboronic acid (1.5 $\mathrm{mg}, 12 \mu \mathrm{mol})$, palladium acetate $(1.0 \mathrm{mg}, 4.5 \mu \mathrm{mol})$, and 2-dicyclohexylphosphino-2'-(N,Ndimethylamino)-biphenyl (Dave-Phos, $2.6 \mathrm{mg}, 6.6 \mu \mathrm{mol}$, Strem Chemicals) together as a single portion of a solid mixture. The vial was purged with $\mathrm{N}_{2}$ for $1 \mathrm{~min}$, then dioxane $(70 \mu \mathrm{L})$ was added via syringe. The rubber septum was removed and the vial was sealed with 2-ply teflon tape, followed by a plastic screw-cap which was finally sealed with parafilm. The red/orange suspension was allowed to stir at $\mathrm{rt}$ for $28 \mathrm{~h}$. Saturated aq. $\mathrm{NaHCO}_{3}(1 \mathrm{~mL})$ was added and the mixture was extracted with EtOAc $(4 \times 1 \mathrm{~mL})$. The combined organic layers were dried over anhydrous $\mathrm{Na}_{2} \mathrm{SO}_{4}$, filtered and concentrated under stream of $\mathrm{N}_{2}$. The residue was flashed through a plug of silica $(50 \rightarrow 100 \%$ EtOAc / pentane). All fractions containing product were further purified on reverse phase HPLC (see below for conditions) to give $1.6 \mathrm{mg}$ of $\mathbf{1 1}(53 \%)$ as a white amorphous solid.

Data for 11:

$\mathbf{R}_{f}=0.53$ (70\% EtOAc, 30\% pentane) - one black spot $\mathrm{w} / \mathrm{p}$-anisaldehyde stain.

RP-HPLC: $\quad$ Retention Time $=35.10 \mathrm{~min}$.

Method: Analytical column; $\mathrm{MeCN} / \mathrm{H}_{2} \mathrm{O}$ at $1.3 \mathrm{~mL} / \mathrm{min}$ start\%(hold time) $\rightarrow$ rate $\rightarrow$ end $\%$ (hold time)

$65 \%(6.4 \mathrm{~min}) \rightarrow 0.52 \% / \mathrm{min} \rightarrow 75 \%(0 \mathrm{~min}) \rightarrow 1.55 \% / \mathrm{min} \rightarrow 90 \%(0 \mathrm{~min})$

IR (thin film): 3460, 3322 (br), 2925, 2854, 1728, 1665, 1454, 1373, 1286, 1259, 1231, 1159, $1135,1104,1082,1005,980 \mathrm{~cm}^{-1}$.

${ }^{1}$ HNMR $\left(600 \mathrm{MHz}, \mathrm{CDCl}_{3}\right): \delta 7.57(4 \mathrm{H}, \mathrm{m}, \mathrm{Ar}-\mathbf{H}), 7.44(2 \mathrm{H}, \mathrm{m}, \mathrm{Ar}-\mathbf{H}), 7.34(3 \mathrm{H}, \mathrm{m}, \mathrm{Ar}-\mathbf{H})$, $6.02(1 \mathrm{H}, \mathrm{d}, J=12.7 \mathrm{~Hz}, \mathrm{C} 13), 6.01(1 \mathrm{H}, \mathrm{d}, J=1.3 \mathrm{~Hz}, \mathrm{C} 23), 5.46(1 \mathrm{H}, \mathrm{dd}, J=7.7,16.2 \mathrm{~Hz}$, C12), $5.41(1 \mathrm{H}, \mathrm{ddt}, J=6.0,12.0,3.0 \mathrm{~Hz}, \mathrm{C} 21), 5.17(1 \mathrm{H}, \mathrm{s}, \mathrm{C} 16), 5.13(1 \mathrm{H}, \mathrm{s}, \mathrm{C} 15-\mathrm{OH}), 5.12$ $(1 \mathrm{H}, \mathrm{d}, J=7.5 \mathrm{~Hz}, \mathrm{C} 11), 4.44(1 \mathrm{H}, \mathrm{d}, J=11.9 \mathrm{~Hz}, \mathrm{C} 3-\mathrm{OH}), 4.41(1 \mathrm{H}, \mathrm{dd}, J=2.9,11.6 \mathrm{~Hz}, \mathrm{C} 6)$, $4.30(1 \mathrm{H}, \mathrm{m}, \mathrm{C} 3), 4.09(3 \mathrm{H}, \mathrm{m}, \mathrm{C} 8, \mathrm{C} 10 \& \mathrm{C} 19), 3.92(1 \mathrm{H}, \mathrm{dt}, J=2.6,12.2 \mathrm{~Hz}, \mathrm{C} 10), 3.87(1 \mathrm{H}$, dd, $J=3.1,11.9 \mathrm{~Hz}, \mathrm{C} 22), 3.73(1 \mathrm{H}, \mathrm{dd}, J=2.4,13.9 \mathrm{~Hz}, \mathrm{C} 18), 3.68(3 \mathrm{H}, \mathrm{s},-\mathrm{OMe}), 3.67(1 \mathrm{H}$, dd, $J=5.8,12.1 \mathrm{~Hz}, \mathrm{C} 22), 3.51(1 \mathrm{H}, \mathrm{ddd}, J=1.5,5.1,9.8 \mathrm{~Hz}, \mathrm{C} 5), 3.35(1 \mathrm{H}, \mathrm{ddd}, J=2.7,10.0$, $12.9 \mathrm{~Hz}, \mathrm{C} 5), 2.60(1 \mathrm{H}, \mathrm{dd}, J=11.7,12.7 \mathrm{~Hz}, \mathrm{C} 2), 2.53(1 \mathrm{H}, \mathrm{dd}, J=2.5,12.5 \mathrm{~Hz}, \mathrm{C} 2), 2.30(2 \mathrm{H}$, m, C24), $2.26(1 \mathrm{H}, \mathrm{m}, \mathrm{C} 4), 2.19(1 \mathrm{H}, \mathrm{ddd}, J=7.3,11.7,15.6 \mathrm{~Hz}, \mathrm{C} 7), 2.06(1 \mathrm{H}, \mathrm{ddd}, J=2.4$, $11.5,13.9 \mathrm{~Hz}, \mathrm{C} 18), 2.02(1 \mathrm{H}, \mathrm{ddd}, J=2.7,12.0,14.1 \mathrm{~Hz}, \mathrm{C} 20), 1.82(1 \mathrm{H}$, ddd, $J=2.4,11.2$, $13.9 \mathrm{~Hz}, \mathrm{C} 20), 1.77(1 \mathrm{H}, \mathrm{dq}, J=4.4,12.9 \mathrm{~Hz}, \mathrm{C} 9), 1.62(3 \mathrm{H}, \mathrm{m}, \mathrm{C} 7 \& \mathrm{C} 25), 1.50(1 \mathrm{H}, \mathrm{d}$ (br), $J=$ $15.8 \mathrm{~Hz}, \mathrm{C} 4), 1.41(1 \mathrm{H}, \mathrm{d}(\mathrm{br}), J=13.1 \mathrm{~Hz}, \mathrm{C} 9), 1.27(8 \mathrm{H}, \mathrm{m}, \mathrm{C} 26-\mathrm{C} 29), 1.21\left(3 \mathrm{H}, \mathrm{s},-\mathrm{C}\left(\mathrm{CH}_{3}\right)_{2}\right)$, $1.06\left(3 \mathrm{H}, \mathrm{s},-\mathrm{C}\left(\mathrm{CH}_{3}\right)_{2}\right), 0.87(3 \mathrm{H}, \mathrm{t}, J=7.2 \mathrm{~Hz}, \mathrm{C} 30)$. 
${ }^{13}$ CNMR (150 MHz, $\mathrm{CDCl}_{3}$, values obtained from 2D HSQC \& HMBC experiments): $\delta$ 172.4, 172.1, 167.0, 151.6, 142.5, 141.0, 140.5, 140.1, 128.8 (2C), 127.4 (2C), 127.4, 127.0 (2C), 126.8 (2C), 126.0, 119.9, 102.3, 99.0, 83.8, 74.5, 74.1, 71.6, 68.1, 66.3, 65.8 (2C), 64.6, 51.1, 45.6, 45.1, 42.2, 36.0, 34.7, 33.7, 32.5, 31.8, 31.1, 29.0 (2C), 24.7, 24.4, 22.5, 19.5, 14.2.

HRMS (MALDI): Calculated for $\mathrm{C}_{47} \mathrm{H}_{64} \mathrm{O}_{13} \mathrm{Na}[\mathrm{M}+\mathrm{Na}]$ : 859.4239. Found: 859.4221.

$[\alpha]_{D}^{25}=-39.1^{\circ}\left(c=0.09, \mathrm{CHCl}_{3}\right)$.

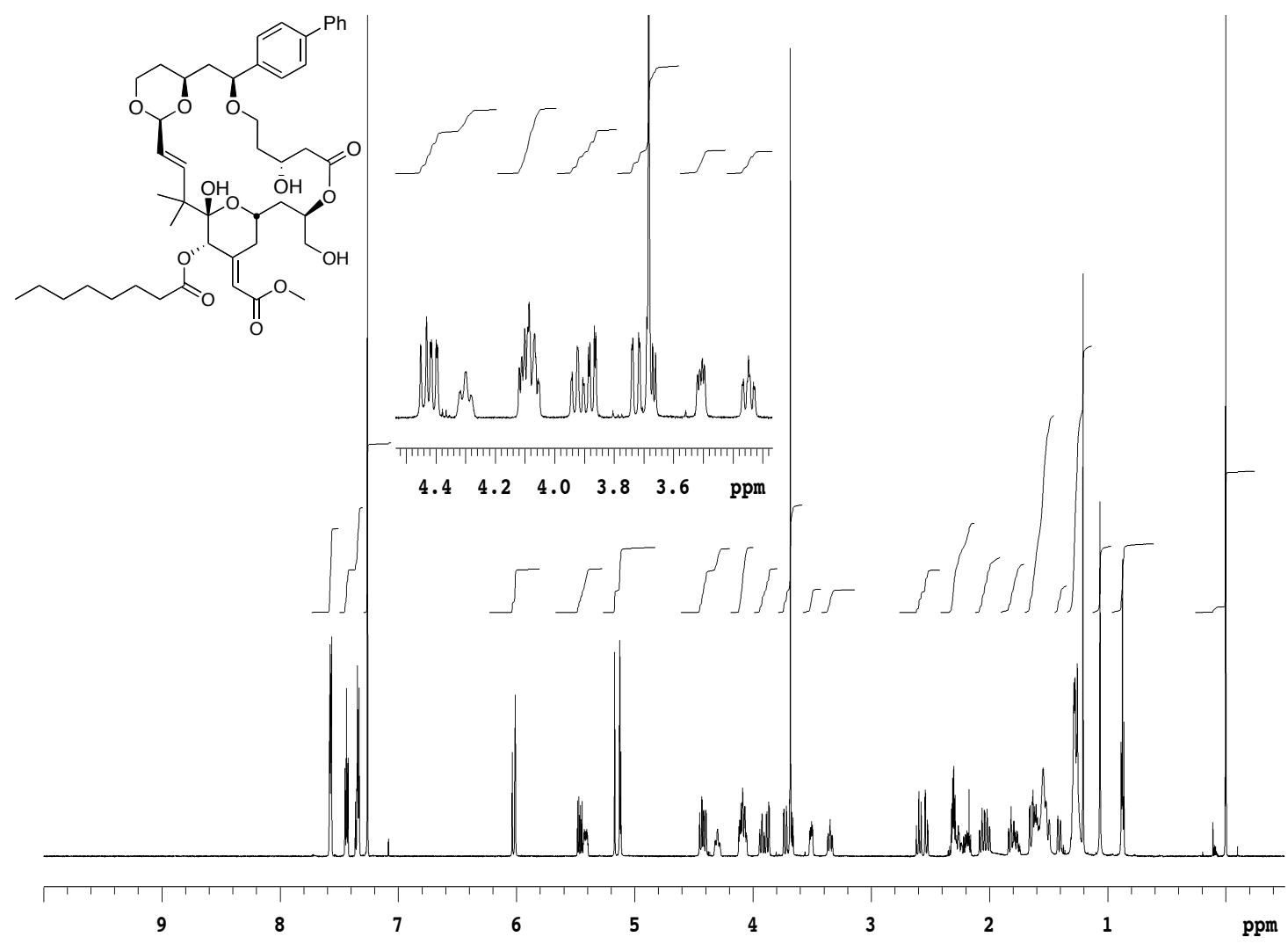




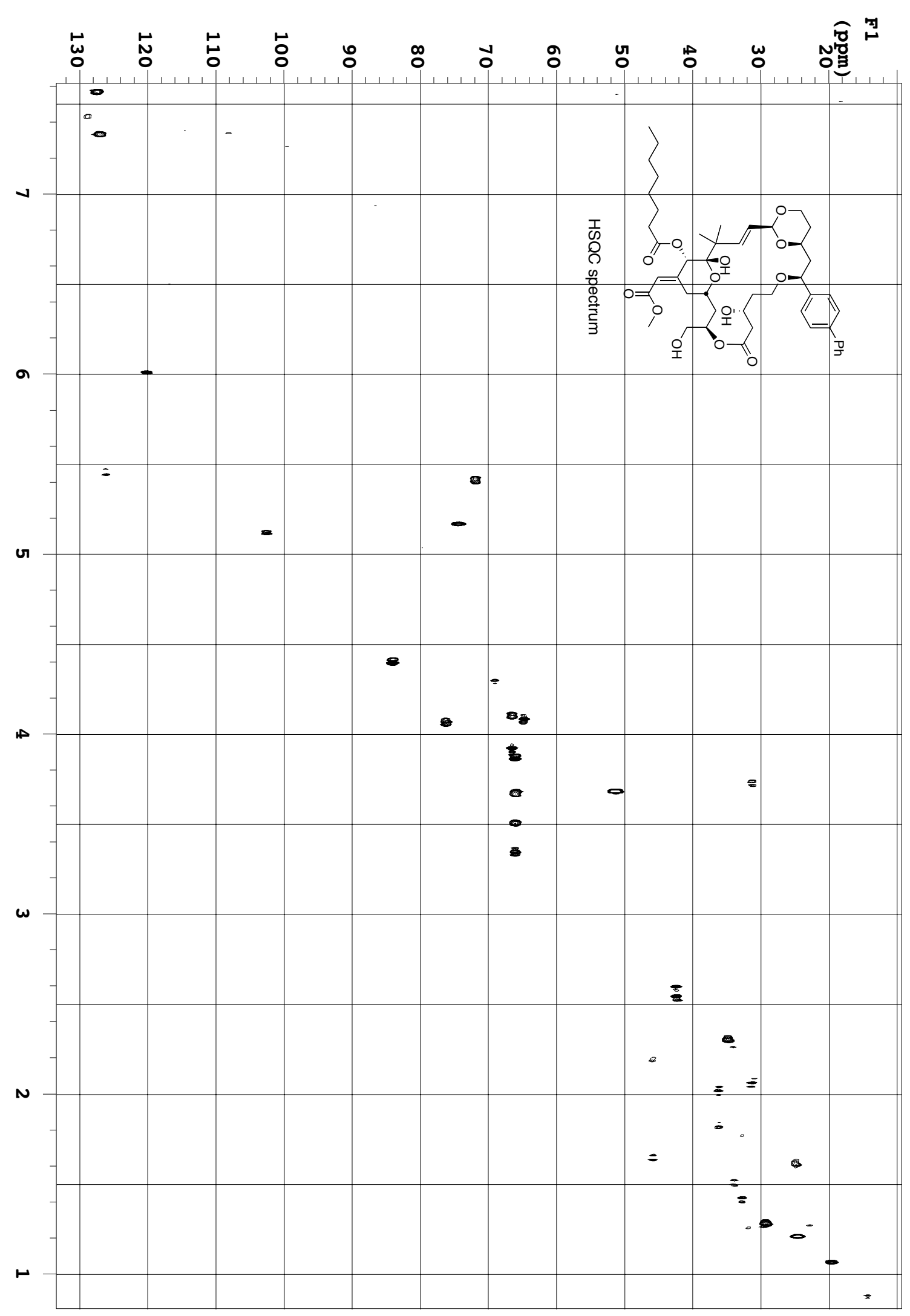




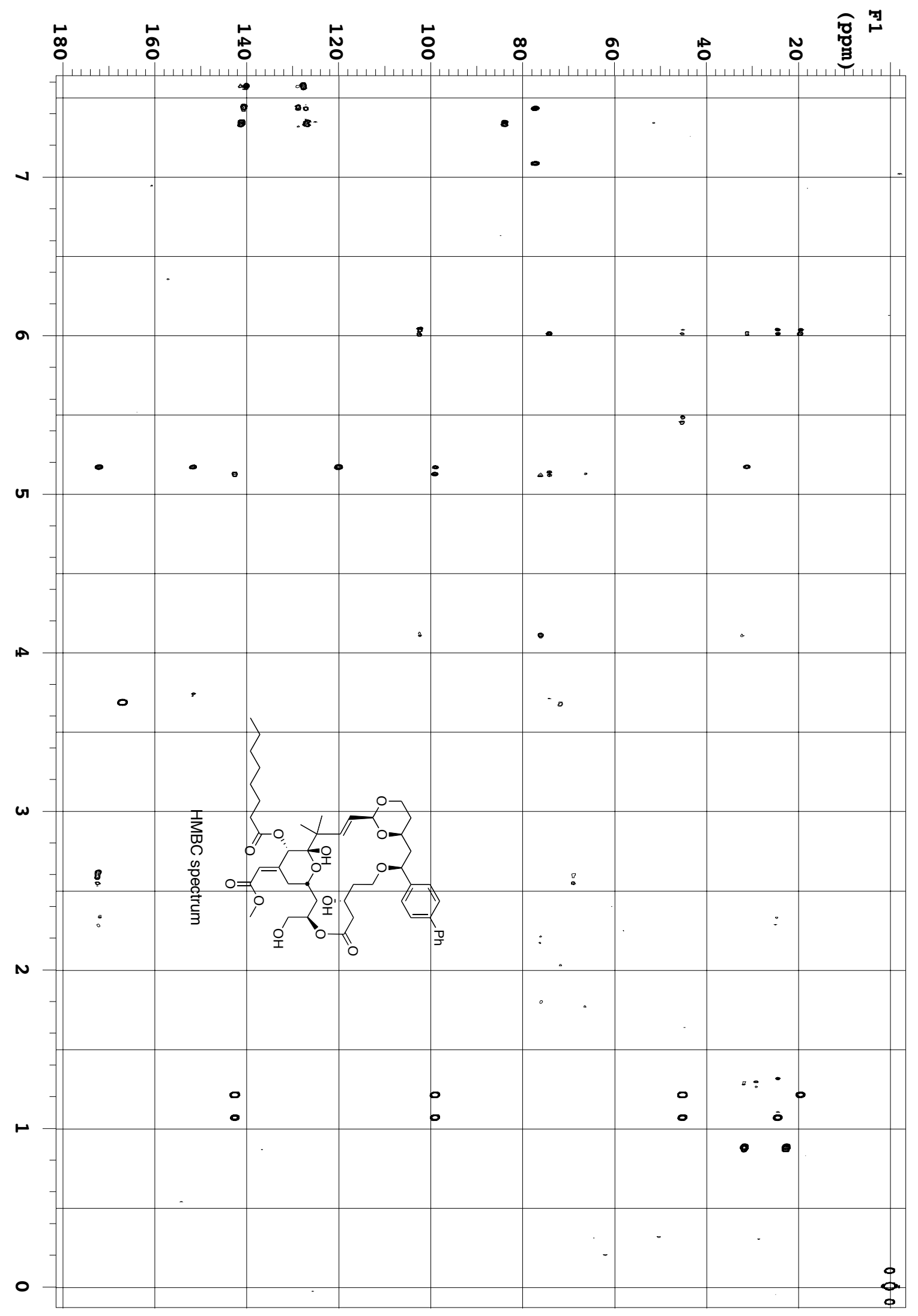



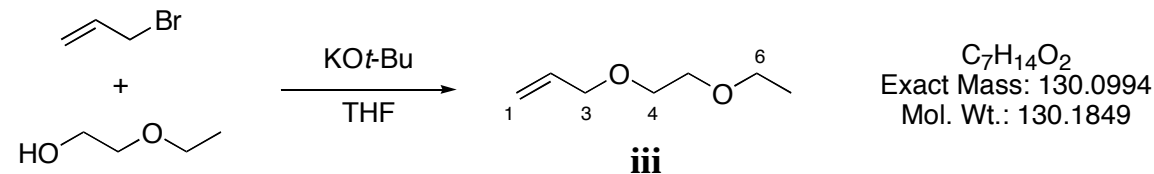

To a solution of 2-ethoxyethanol $(2.0 \mathrm{~mL}, 20.6 \mathrm{mmol})$ in THF $(50 \mathrm{~mL})$ at $\mathrm{rt}$ under $\mathrm{N}_{2}$, was added solid potassium tert-butoxide $(4.63 \mathrm{~g}, 41.3 \mathrm{mmol})$ in one portion. The white suspension was stirred for $15 \mathrm{~min}$ then allyl bromide $(2.6 \mathrm{~mL}, 31.0 \mathrm{mmol})$ was added dropwise over $2 \mathrm{~min}$ via syringe. The suspension was stirred for $1 \mathrm{~h}$, over which time the reaction turned a light brown color. The reaction mixture was poured into sat. aq. $\mathrm{NH}_{4} \mathrm{Cl}(60 \mathrm{~mL})$ and the layers were separated. The aqueous layer was extracted with $\mathrm{Et}_{2} \mathrm{O}(2 \times 30 \mathrm{~mL})$ and the combined organic layers were dried over anhydrous $\mathrm{MgSO}_{4}$, filtered and concentrated to give a yellow liquid. Flash chromatography on silica (15\% EtOAc / pentane) gave $626 \mathrm{mg}$ of 3-(2-ethoxy-ethoxy)-prop-1-ene (iii) as a colorless liquid (16\%). A significant amount of material was lost while removing residual solvent under high vacuum due to the volatility of the product.

Data for iii:

$\mathbf{R}_{f}=0.49$ (10\% EtOAc, $90 \%$ pentane $)-$ one spot, $\mathrm{KMnO}_{4}$ stain.

GC: $\quad$ Retention Time $=9.42 \min (>98 \%$ purity $)$.

Method: start $^{\circ}$ (time) $\rightarrow$ rate $\rightarrow$ end $^{\circ}$ (time)

$40^{\circ}(5 \mathrm{~min}) \rightarrow 5^{\circ} / \mathrm{min} \rightarrow 70^{\circ}(0 \mathrm{~min}) \rightarrow 20^{\circ} / \mathrm{min} \rightarrow 150^{\circ}(2 \mathrm{~min})$

IR (thin film): 3083, 2977, 2866, 1642, 1445, 1373, 1350, 1293, 1247, 1121, 1052, $996 \mathrm{~cm}^{-1}$.

${ }^{1}$ HNMR (400 MHz, $\left.\mathrm{CDCl}_{3}\right): \delta 5.93(1 \mathrm{H}, \mathrm{ddt}, J=10.6,17.1,5.7 \mathrm{~Hz}, \mathrm{C} 2), 5.28(1 \mathrm{H}, \mathrm{m}, \mathrm{C} 1), 5.19$ $(1 \mathrm{H}, \mathrm{m}, \mathrm{C} 1), 4.04(2 \mathrm{H}, \mathrm{m}, \mathrm{C} 3), 3.60(4 \mathrm{H}, \mathrm{s}, \mathrm{C} 4 \& \mathrm{C} 5), 3.54(2 \mathrm{H}, \mathrm{q}, J=7.0 \mathrm{~Hz}, \mathrm{C} 6), 1.23(3 \mathrm{H}, \mathrm{t}, J$ $=7.0 \mathrm{~Hz}, \mathrm{C} 7)$.

${ }^{13}$ CNMR (100 MHz, $\mathrm{CDCl}_{3}$ ): $\delta$ 134.8, 117.2, 72.3, 69.8, 69.4, 66.7, 15.1 .

HRMS (EI+): Calculated for $\mathrm{C}_{7} \mathrm{H}_{15} \mathrm{O}_{2}[\mathrm{M}+\mathrm{H}]:$ 131.1072. Found: 131.1068 . 

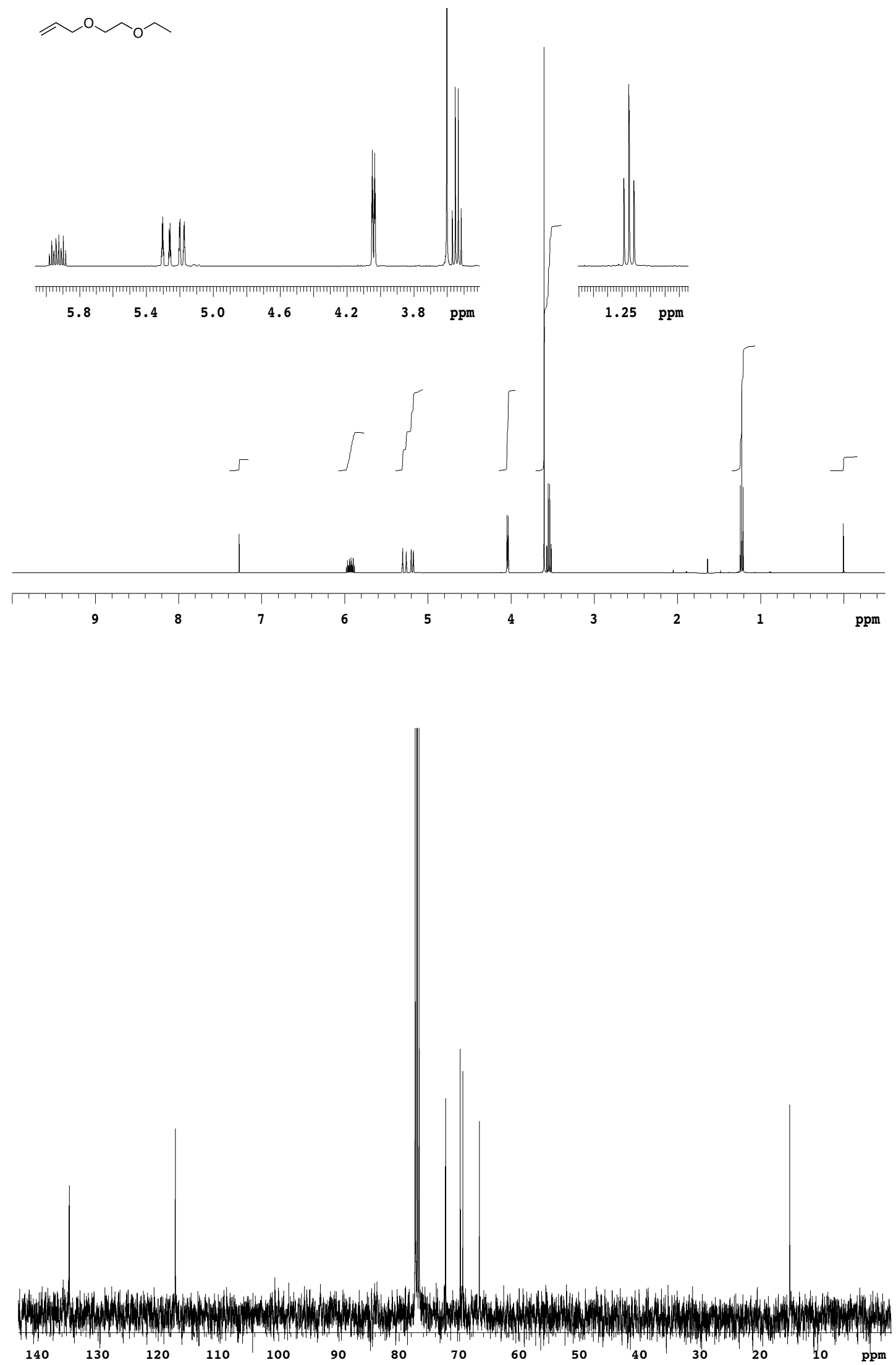

S-31 


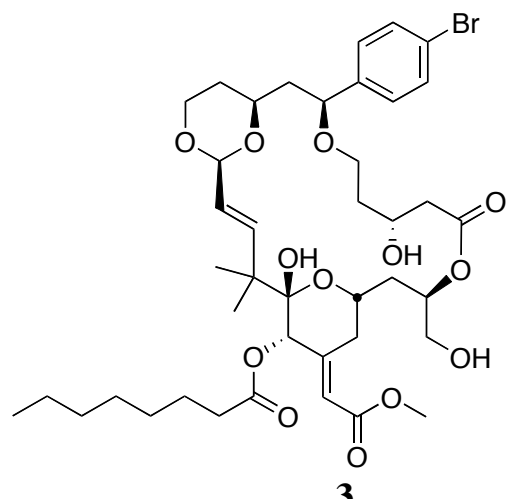

3

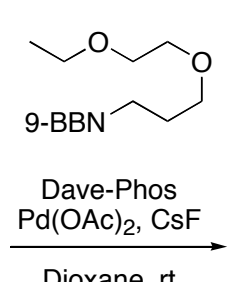

Dioxane, rt

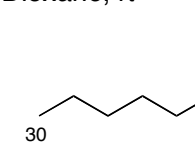

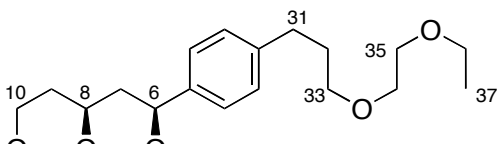

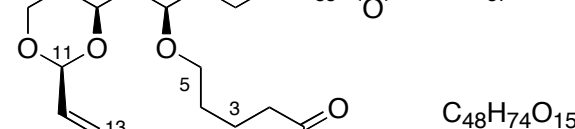

Exact Mass: 890.5028

Mol. Wt.: 891.0922

C, $64.70 ; \mathrm{H}, 8.37 ; \mathrm{O}, 26.93$

A stock solution of the alkylborane was prepared as follows: 3-(2-ethoxy-ethoxy)-propene (iii) $(20 \mu \mathrm{L}, 0.15 \mathrm{mmol})$ was measured into a dried $4 \mathrm{~mL}$ vial under $\mathrm{N}_{2}$. The vial was cooled to 0 ${ }^{\circ} \mathrm{C}$ in an ice bath and 9-BBN ( $310 \mu \mathrm{L}$ of a $0.5 \mathrm{M}$ solution, $\left.0.15 \mathrm{mmol}\right)$ was added in a single portion via syringe. The bath was removed and the solution allowed to stir for $4.5 \mathrm{~h}$.

Aryl bromide $3(2.8 \mathrm{mg}, 3.3 \mu \mathrm{mol})$ and $\mathrm{CsF}(4.4 \mathrm{mg}, 29 \mu \mathrm{mol})$ were measured into a dry 2 $\mathrm{mL}$ glass vial and placed under high vacuum for $1.5 \mathrm{~h}$. The vial was removed from the vacuum under $\mathrm{N}_{2}$ gas and placed under an $\mathrm{N}_{2}$ atmosphere. To this vial was added palladium acetate (2.0 $\mathrm{mg}, 9.0 \mu \mathrm{mol}$ ), and 2-dicyclohexylphosphino-2'-(N,N-dimethylamino)-biphenyl (Dave-Phos, 5.2 $\mathrm{mg}, 13 \mu \mathrm{mol}$, Strem Chemicals) together as a single portion of a solid mixture. The vial was purged with $\mathrm{N}_{2}$ for $1 \mathrm{~min}$, then dioxane $(70 \mu \mathrm{L})$ was added via syringe followed by an aliquot of the stock alkyl-borane solution $(25 \mu \mathrm{L}, 11 \mu \mathrm{mol})$. The rubber septum was removed and the vial was sealed with 2-ply teflon tape, followed by a plastic screw-cap which was finally sealed with parafilm. The vial was vortexed momentarily (to wash solids on the vial walls into the solvent) and the light orange suspension was allowed to stir at $\mathrm{rt}$ for $53.8 \mathrm{~h}$. Saturated aq. $\mathrm{NaHCO}_{3}(1 \mathrm{~mL})$ was added and the mixture was extracted with EtOAc $(6 \times 1 \mathrm{~mL})$. The combined organic layers were dried over anhydrous $\mathrm{Na}_{2} \mathrm{SO}_{4}$, filtered and concentrated under stream of $\mathrm{N}_{2}$. The residue was flashed through a plug of silica $(50 \rightarrow 100 \%$ EtOAc / pentane). All fractions containing product were further purified on reverse phase HPLC $\left(65 \rightarrow 90 \% \mathrm{MeCN} / \mathrm{H}_{2} \mathrm{O}\right)$ to give $0.6 \mathrm{mg}$ of the hydrogenolysis product (24\%) and $0.6 \mathrm{mg}$ of $\mathbf{1 2}(20 \%)$ as a white amorphous solid.

Data for 12:

$\mathbf{R}_{f}=0.39$ (80\% EtOAc / pentane $)-$ one black spot w/ p-anisaldehyde stain.

RP-HPLC: $\quad$ Retention Time $=35.52 \mathrm{~min}$.

Method: Analytical column; $\mathrm{MeCN} / \mathrm{H}_{2} \mathrm{O}$ at $1.3 \mathrm{~mL} / \mathrm{min}$ start $\%$ (hold time) $\rightarrow$ rate $\rightarrow$ end $\%$ (hold time)

$65 \%(6.4 \mathrm{~min}) \rightarrow 0.52 \% / \mathrm{min} \rightarrow 75 \%(0 \mathrm{~min}) \rightarrow 1.55 \% / \mathrm{min} \rightarrow 90 \%(0$

$\mathrm{min}) \rightarrow-10.42 \% / \mathrm{min} \rightarrow 65 \%(2.47 \mathrm{~min})$

IR (thin film): 3460, 3330 (br), 2927, 2858, 1727, 1433, 1402, 1378, 1362, 1287, 1258, 1230, $1159,1135,1107,1005,980 \mathrm{~cm}^{-1}$.

${ }^{1}$ HNMR $\left(600 \mathrm{MHz}, \mathrm{CDCl}_{3}\right): \delta 7.16(4 \mathrm{H}, \mathrm{s}, \mathrm{Ar}-\mathrm{H}), 6.00(1 \mathrm{H}, \mathrm{d}, J=15.9 \mathrm{~Hz}, \mathrm{C} 13), 6.00(1 \mathrm{H}, \mathrm{d}, J$ $=2.0 \mathrm{~Hz}, \mathrm{C} 23), 5.44(1 \mathrm{H}, \mathrm{dd}, J=7.6,16.1 \mathrm{~Hz}, \mathrm{C} 12), 5.40(1 \mathrm{H}, \mathrm{ddt}, J=6.0,12.0,3.0 \mathrm{~Hz}, \mathrm{C} 21)$, $5.15(1 \mathrm{H}, \mathrm{s}, \mathrm{C} 16), 5.11(1 \mathrm{H}, \mathrm{s}, \mathrm{C} 15-\mathrm{OH}), 5.09(1 \mathrm{H}, \mathrm{d}, J=7.6 \mathrm{~Hz}, \mathrm{C} 11), 4.42(1 \mathrm{H}, \mathrm{d}, J=11.7 \mathrm{~Hz}$, C3-OH), $4.31(1 \mathrm{H}, \mathrm{dd}, J=2.7,11.7 \mathrm{~Hz}, \mathrm{C} 6), 4.27(1 \mathrm{H}, \mathrm{m}, \mathrm{C} 3), 4.05(3 \mathrm{H}, \mathrm{m}, \mathrm{C} 8, \mathrm{C} 10$ \& C19), $3.90(1 \mathrm{H}, \mathrm{dt}, J=2.2,11.8 \mathrm{~Hz}, \mathrm{C} 10), 3.86(1 \mathrm{H}, \mathrm{ddd}, J=2.9,5.1,11.9 \mathrm{~Hz}, \mathrm{C} 22), 3.71(1 \mathrm{H}, \mathrm{dd}, J=$ 2.2, $13.7 \mathrm{~Hz}, \mathrm{C} 18), 3.67(3 \mathrm{H}, \mathrm{s},-\mathrm{OMe}), 3.66(1 \mathrm{H}$, pentet, $J=6.1 \mathrm{~Hz}, \mathrm{C} 22), 3.58(4 \mathrm{H}, \mathrm{s}, \mathrm{C} 34$ \& C35), $3.53(2 \mathrm{H}, \mathrm{q}, J=7.0 \mathrm{~Hz}, \mathrm{C} 36), 3.47(2 \mathrm{H}, \mathrm{t}, J=6.6 \mathrm{~Hz}, \mathrm{C} 33), 3.42(1 \mathrm{H}, \mathrm{m}, \mathrm{C} 5), 3.28(1 \mathrm{H}$, 
ddd, $J=2.7,9.7,13.2 \mathrm{~Hz}, \mathrm{C} 5), 2.67(2 \mathrm{H}, \mathrm{t}, J=7.8 \mathrm{~Hz}, \mathrm{C} 31), 2.57(1 \mathrm{H}, \mathrm{dd}, J=12.0,12.3 \mathrm{~Hz}$, C2), $2.50(1 \mathrm{H}, \mathrm{dd}, J=2.4,12.5 \mathrm{~Hz}, \mathrm{C} 2), 2.30(2 \mathrm{H}, \mathrm{dt}, J=4.4,7.5 \mathrm{~Hz}, \mathrm{C} 24), 2.21(1 \mathrm{H}, \mathrm{m}, \mathrm{C} 4)$, $2.13(1 \mathrm{H}$, ddd, $J=7.4,11.6,15.9 \mathrm{~Hz}, \mathrm{C} 7), 2.05(1 \mathrm{H}, \mathrm{ddd}, J=2.2,11.5,13.7 \mathrm{~Hz}, \mathrm{C} 18), 2.00(1 \mathrm{H}$, m, C20), $1.98(1 \mathrm{H}, \mathrm{m}, \mathrm{C} 22-\mathrm{OH}), 1.90(2 \mathrm{H}, \mathrm{dq}, J=8.5,6.5 \mathrm{~Hz}, \mathrm{C} 32), 1.80(1 \mathrm{H}, \mathrm{ddd}, J=2.8,11.4$, $14.0 \mathrm{~Hz}, \mathrm{C} 20), 1.75(1 \mathrm{H}, \mathrm{dq}, J=4.9,12.4 \mathrm{~Hz}, \mathrm{C} 9), 1.59$ (3H, m, C7 \& C25), 1.47 (1H, d (br), $J=$ $14.6 \mathrm{~Hz}, \mathrm{C} 4), 1.38(1 \mathrm{H}, \mathrm{d}(\mathrm{br}), J=13.1 \mathrm{~Hz}, \mathrm{C} 9), 1.26(8 \mathrm{H}, \mathrm{m}, \mathrm{C} 26-\mathrm{C} 29), 1.21(3 \mathrm{H}, \mathrm{t}, J=6.8 \mathrm{~Hz}$, C37), 1.20 (3H, s, -C(CH$\left.)_{2}\right), 1.05\left(3 \mathrm{H}, \mathrm{s},-\mathrm{C}\left(\mathrm{CH}_{3}\right)_{2}\right), 0.87(3 \mathrm{H}, \mathrm{t}, J=7.0 \mathrm{~Hz}, \mathrm{C} 30)$.

${ }^{13}$ CNMR (150 MHz, $\mathrm{CDCl}_{3}$, values obtained from 2D HSQC \& HMBC experiments): $\delta$ 172.4, 172.1, 166.9, 151.6, 142.5, 142.0, 138.6, 128.6 (2C), 126.2 (2C), 126.1, 119.7, 102.2, 99.0, 83.8, 75.9, 74.0, 71.5, 70.4, 69.9 (2C), 68.7, 66.6, 66.2, 65.7, 65.5, 64.5, 51.0, 45.5, 45.1, 42.0, 35.8, 34.5, 33.7, 32.3, 31.9, 31.6, 30.9 (2C), 30.1, 29.5, 25.1, 24.8, 23.1, 19.9, 15.7, 14.5 .

HRMS (MALDI): Calculated for $\mathrm{C}_{48} \mathrm{H}_{74} \mathrm{O}_{15} \mathrm{Na}$ [M+Na]: 913.4920. Found: 913.4883.

$[\alpha]_{D}^{23}=-87.5^{\circ}\left(c=0.02, \mathrm{CHCl}_{3}\right)$.

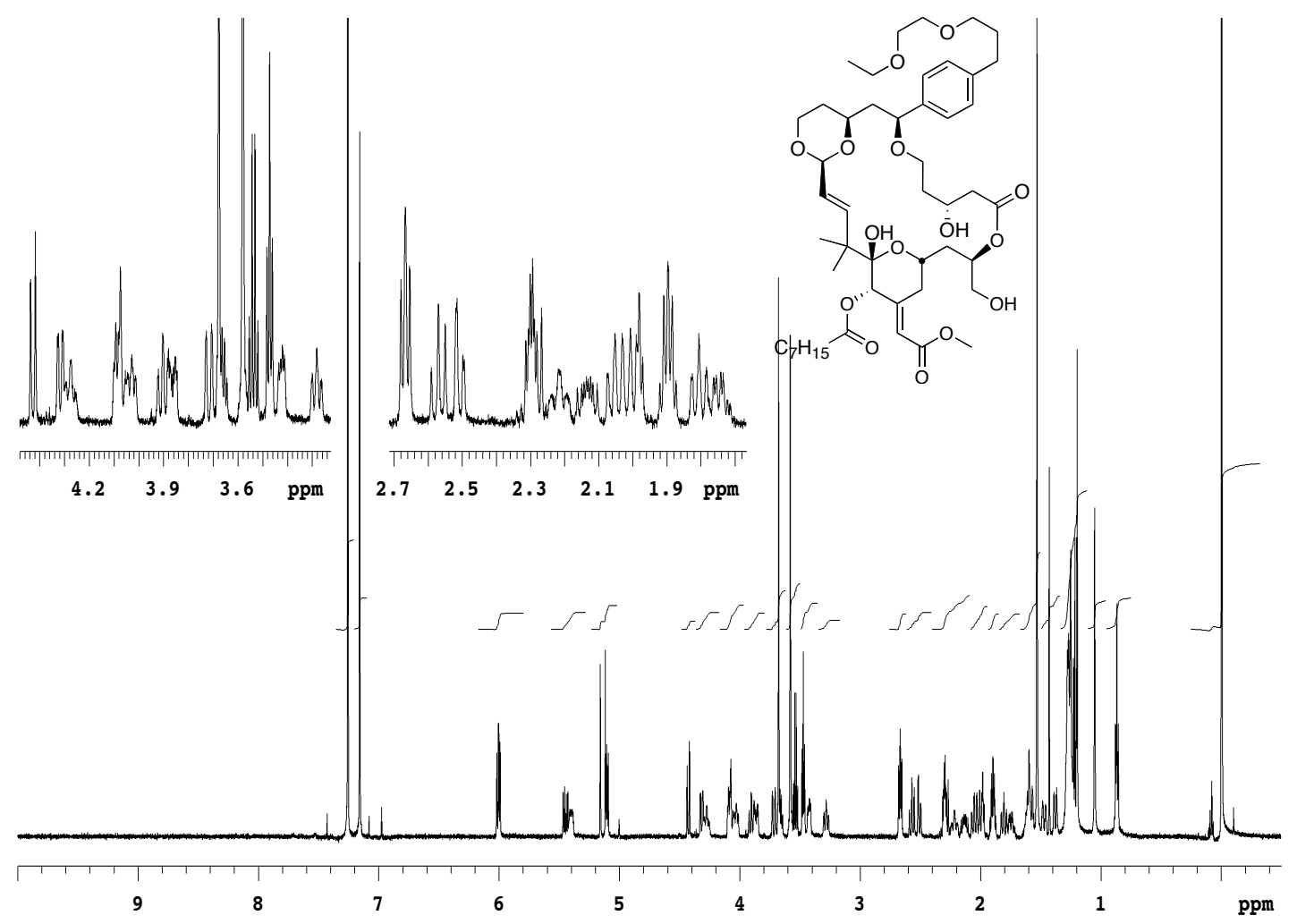




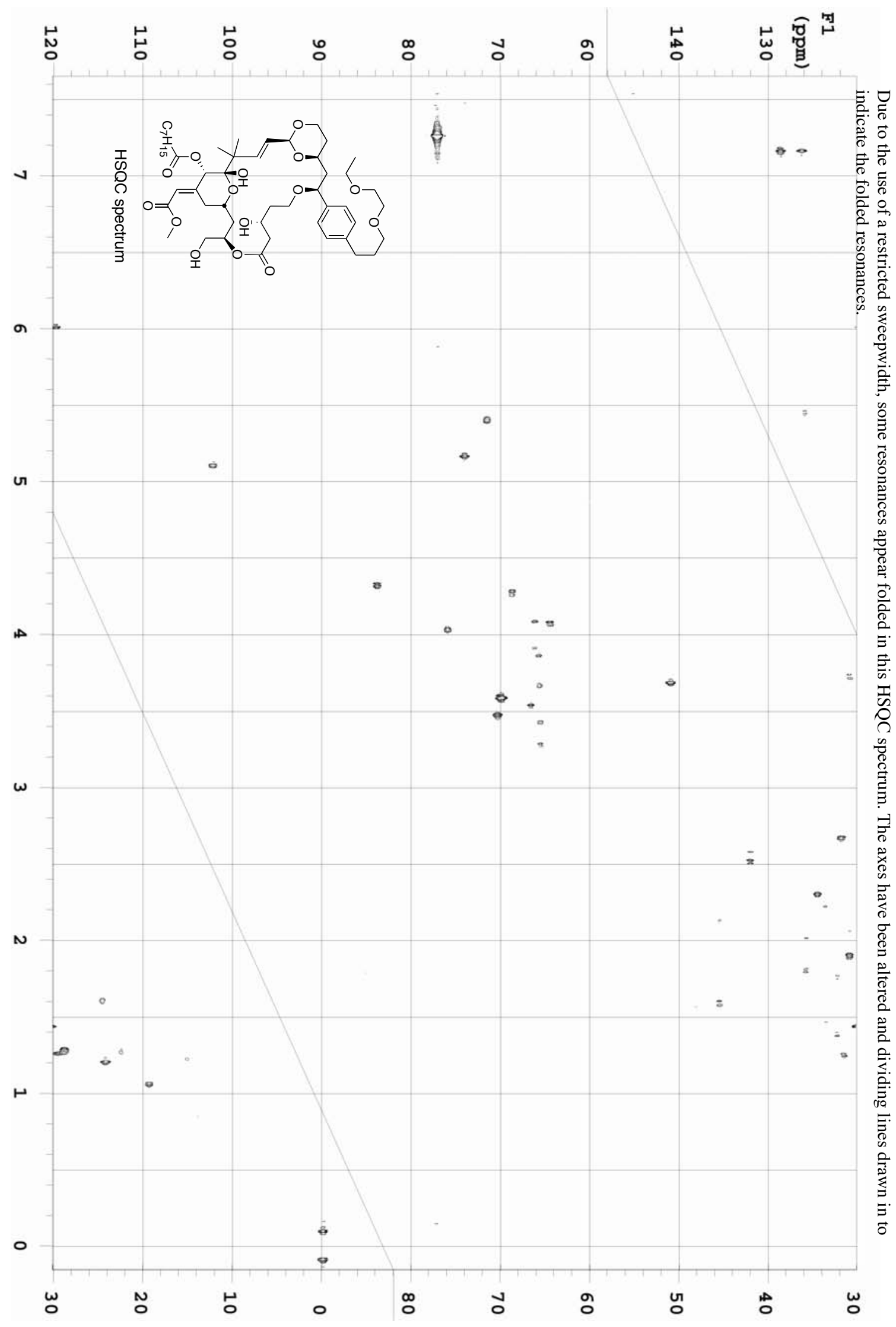




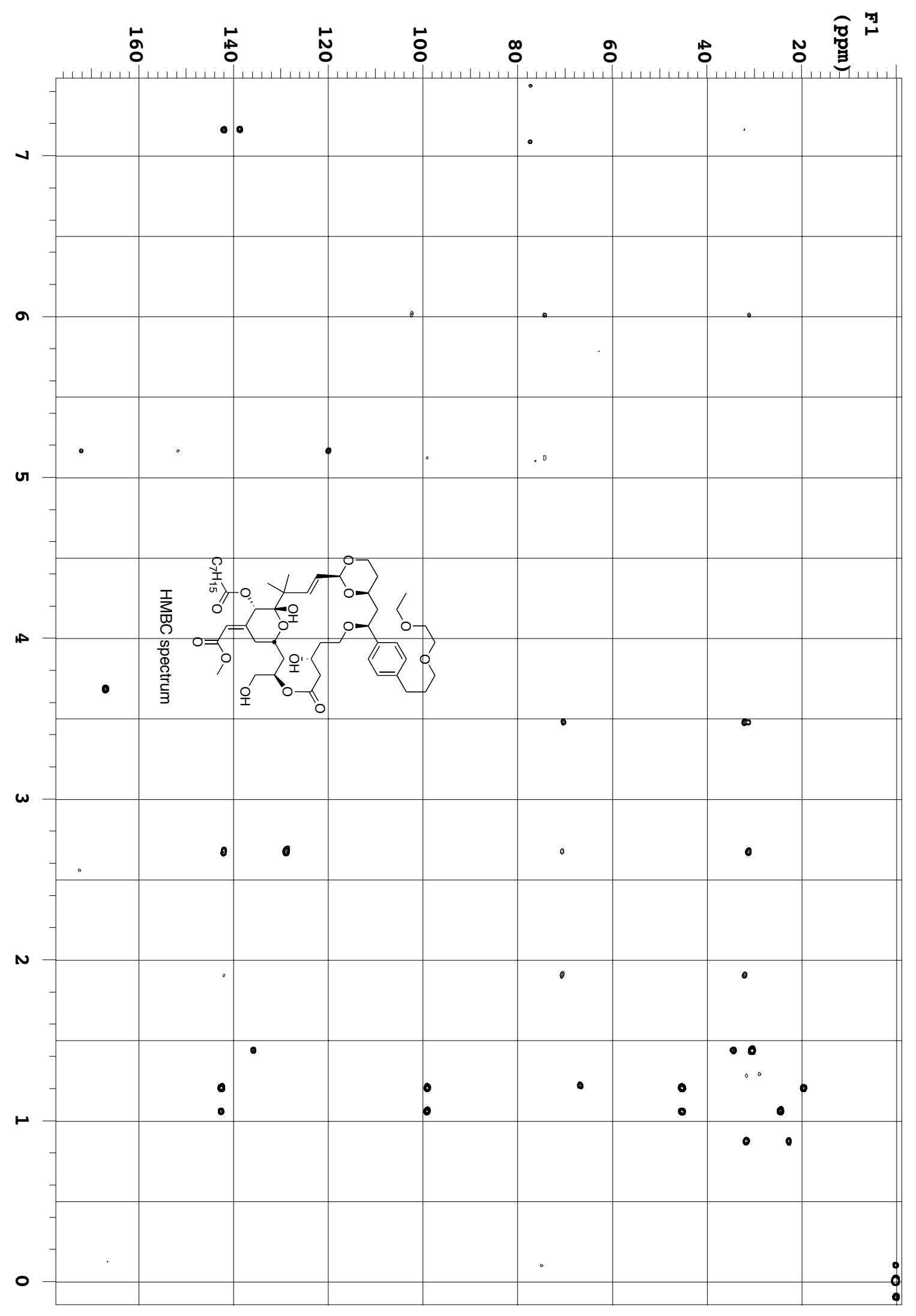




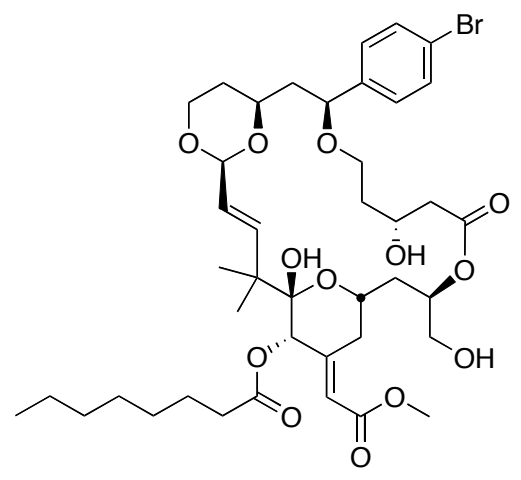

3

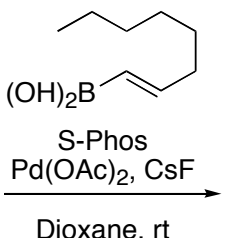

Dioxane, rt

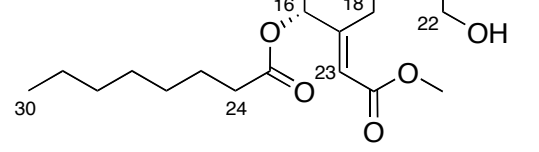

13

Aryl bromide $3(3.0 \mathrm{mg}, 3.6 \mu \mathrm{mol})$ and $\mathrm{CsF}(3.6 \mathrm{mg}, 24 \mu \mathrm{mol})$ were measured into a $2 \mathrm{~mL}$ glass vial and placed under high vacuum for $20 \mathrm{~min}$. The vial was removed from the vacuum under $\mathrm{N}_{2}$ gas and placed under an $\mathrm{N}_{2}$ atmosphere. To this vial was added palladium acetate (1.0 $\mathrm{mg}, 4.5 \mu \mathrm{mol})$, trans-1-octen-1-ylboronic acid $(1.1 \mathrm{mg}, 7.0 \mu \mathrm{mol}$, Aldrich) and 2dicyclohexylphosphino-2',6'-dimethoxy-1,1'-biphenyl (S-Phos, $2.8 \mathrm{mg}, 6.7 \mu \mathrm{mol}$, Strem Chemicals) together as a single portion of a solid mixture. The vial was purged with $\mathrm{N}_{2}$ for 1 min, then dioxane $(70 \mu \mathrm{L})$ was added via syringe. The rubber septum was removed and the vial was sealed with 2-ply teflon tape, followed by a plastic screw-cap which was finally sealed with parafilm. The vial was vortexed momentarily (to wash solids on the vial walls into the solvent) and the dark orange suspension was allowed to stir at $\mathrm{rt}$ for $46 \mathrm{~h}$. The reaction mixture was filtered through a cotton plug using $\mathrm{Et}_{2} \mathrm{O}$ and then concentrated under a stream of $\mathrm{N}_{2}$. Flash chromatography of the residue $(50 \rightarrow 100 \%$ EtOAc / pentane) yielded $1.1 \mathrm{mg}$ of $\mathbf{1 3}(35 \%)$ as well as $1.3 \mathrm{mg}$ of recovered $\mathbf{3}(43 \%)$ both as colorless solid residues ( $62 \%$ at $57 \%$ conversion).

Data for 13:

$\mathbf{R}_{f}=0.63$ (70\% EtOAc, 30\% pentane) - one black spot $\mathrm{w} / \mathrm{p}$-anisaldehyde stain.

IR (thin film): 3460, 3331 (br), 2926, 2855, 1722, 1378, 1362, 1287, 1259, 1231, 1159, 1135, $1105,1082,1063,1005,980,918,886,852,808,755,660 \mathrm{~cm}^{-1}$.

${ }^{1}$ HNMR $\left(600 \mathrm{MHz}, \mathrm{CDCl}_{3}\right): \delta 7.31(2 \mathrm{H}, \mathrm{m}, \mathrm{Ar}-\mathbf{H}), 7.18(2 \mathrm{H}, \mathrm{m}, \mathrm{Ar}-\mathbf{H}), 6.35(1 \mathrm{H}, \mathrm{dt}, J=15.8$, $1.3 \mathrm{~Hz}, \mathrm{C} 31), 6.23(1 \mathrm{H}, \mathrm{dt}, J=15.8,6.9 \mathrm{~Hz}, \mathrm{C} 32), 6.01(1 \mathrm{H}, \mathrm{d}, J=15.9 \mathrm{~Hz}, \mathrm{C} 13), 6.00(1 \mathrm{H}, \mathrm{d}, J$ $=2.1 \mathrm{~Hz}, \mathrm{C} 23), 5.45(1 \mathrm{H}, \mathrm{dd}, J=7.3,15.9 \mathrm{~Hz}, \mathrm{C} 12), 5.40(1 \mathrm{H}, \mathrm{ddt}, J=5.9,11.9,2.8 \mathrm{~Hz}, \mathrm{C} 21)$, $5.16(1 \mathrm{H}, \mathrm{s}, \mathrm{C} 16), 5.12(1 \mathrm{H}, \mathrm{s}, \mathrm{C} 15-\mathrm{OH}), 5.10(1 \mathrm{H}, \mathrm{d}, J=7.3 \mathrm{~Hz}, \mathrm{C} 11), 4.42(1 \mathrm{H}, \mathrm{d}, J=11.8 \mathrm{~Hz}$, C3-OH), $4.32(1 \mathrm{H}, \mathrm{dd}, J=2.7,11.8 \mathrm{~Hz}, \mathrm{C} 6), 4.28(1 \mathrm{H}, \mathrm{m}, \mathrm{C} 3), 4.08(2 \mathrm{H}, \mathrm{m}, \mathrm{C} 10$ \& C19), 4.03 $(1 \mathrm{H}, \mathrm{ddd}, J=2.7,7.3,11.4 \mathrm{~Hz}, \mathrm{C} 8), 3.90(1 \mathrm{H}, \mathrm{dt}, J=2.1,11.8 \mathrm{~Hz}, \mathrm{C} 10), 3.86(1 \mathrm{H}, \mathrm{ddd}, J=3.2$, $5.1,11.9 \mathrm{~Hz}, \mathrm{C} 22), 3.72(1 \mathrm{H}, \mathrm{dd}, J=2.5,13.8 \mathrm{~Hz}, \mathrm{C} 18), 3.68(3 \mathrm{H}, \mathrm{s},-\mathrm{OMe}), 3.67(1 \mathrm{H}$, pent, $J=$ $6.3 \mathrm{~Hz}, \mathrm{C} 22), 3.43(1 \mathrm{H}, \mathrm{ddd}, J=1.7,5.1,9.8 \mathrm{~Hz}, \mathrm{C} 5), 3.29(1 \mathrm{H}, \mathrm{ddd}, J=2.7,9.7,13.2 \mathrm{~Hz}, \mathrm{C} 5)$, $2.57(1 \mathrm{H}, \mathrm{dd}, J=11.6,12.7 \mathrm{~Hz}, \mathrm{C} 2), 2.51(1 \mathrm{H}, \mathrm{dd}, J=2.6,12.7 \mathrm{~Hz}, \mathrm{C} 2), 2.30(2 \mathrm{H}, \mathrm{m}, \mathrm{C} 24), 2.23$ $(1 \mathrm{H}, \mathrm{m}, \mathrm{C} 4), 2.19(2 \mathrm{H}, \mathrm{ddd}, J=1.4,7.1,14.8 \mathrm{~Hz}, \mathrm{C} 33), 2.13(1 \mathrm{H}, \mathrm{ddd}, J=7.4,11.8,15.8 \mathrm{~Hz}$, C7), 2.05 (1H, ddd, $J=2.2,11.6,13.8 \mathrm{~Hz}, \mathrm{C} 18), 2.01(1 \mathrm{H}, \mathrm{ddd}, J=2.0,11.5,14.1 \mathrm{~Hz}, \mathrm{C} 20), 1.99$ $(1 \mathrm{H}, \mathrm{dd}, J=5.1,6.5 \mathrm{~Hz}, \mathrm{C} 22-\mathrm{OH}), 1.81(1 \mathrm{H}, \mathrm{ddd}, J=2.7,11.5,14.2 \mathrm{~Hz}, \mathrm{C} 20), 1.75(1 \mathrm{H}, \mathrm{dq}, J=$ 4.9, $12.7 \mathrm{~Hz}, \mathrm{C} 9), 1.60(3 \mathrm{H}, \mathrm{m}, \mathrm{C} 7 \& \mathrm{C} 25), 1.45(3 \mathrm{H}, \mathrm{m}, \mathrm{C} 4 \& \mathrm{C} 34), 1.38(1 \mathrm{H}, \mathrm{d}(\mathrm{br}), J=13.4$ $\mathrm{Hz}, \mathrm{C} 9), 1.21-1.36\left(14 \mathrm{H}, \mathrm{m}, \mathrm{C} 26-\mathrm{C} 29\right.$ \& 35-37), $1.20\left(3 \mathrm{H}, \mathrm{s},-\mathrm{C}\left(\mathrm{CH}_{3}\right)_{2}\right), 1.05\left(3 \mathrm{H}, \mathrm{s},-\mathrm{C}\left(\mathrm{CH}_{3}\right)_{2}\right)$, $0.89(3 \mathrm{H}, \mathrm{t}, J=7.0 \mathrm{~Hz}, \mathrm{C} 30$ or C38), $0.87(3 \mathrm{H}, \mathrm{t}, J=7.1 \mathrm{~Hz}, \mathrm{C} 30$ or C 38$)$.

${ }^{13}$ CNMR $\left(125 \mathrm{MHz}, \mathrm{CDCl}_{3}\right): \delta 172.5,172.1,167.0,151.6,142.5,139.5,137.9,131.7,129.1$, 126.6 (2C), 126.1 (2C), 126.0, 119.9, 102.3, 98.9, 83.8, 75.9, 74.1, 71.6, 68.8, 66.3, 65.7, 65.6, 
$64.5,51.1,45.4,45.1,42.1,35.9,34.6,33.6,33.0,32.3,31.7,31.6,31.0,29.3,29.0,28.88,28.87$, 24.7, 24.3, 22.62, 22.55, 19.4, 14.11, 14.06 .

HRMS (MALDI): Calculated for $\mathrm{C}_{49} \mathrm{H}_{74} \mathrm{O}_{13} \mathrm{Na}$ : 893.5022. Found: 893.5026.

$[\alpha]_{D}^{24}=-40.9^{\circ}\left(c=0.13, \mathrm{CHCl}_{3}\right)$. 

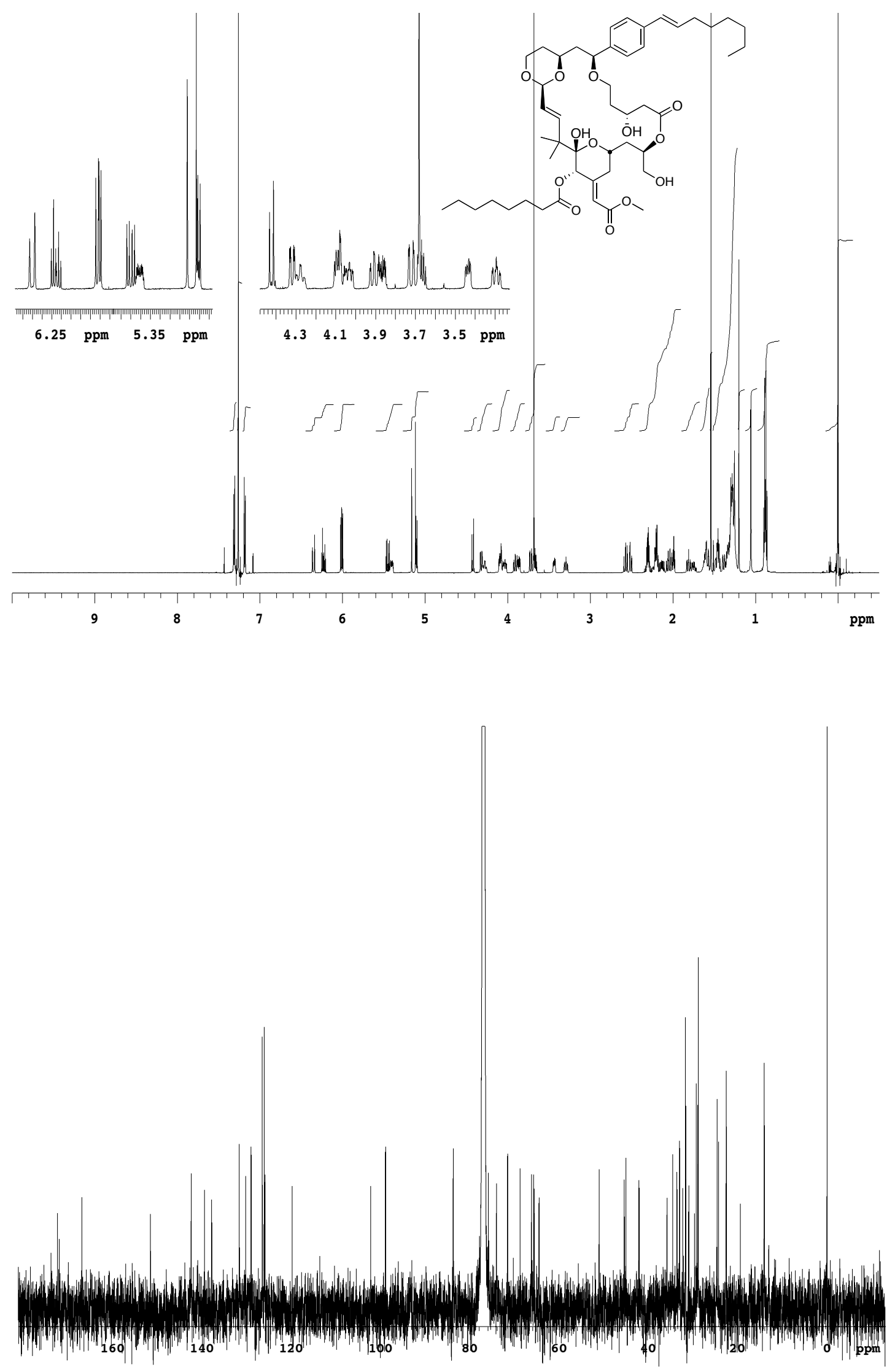

S-38 


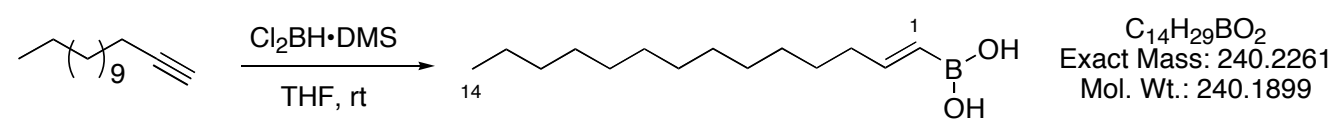

iv

To a solution of tetradecyne $(1.0 \mathrm{~mL}, 4.1 \mathrm{mmol}$, Farchan Laboratories $)$ in THF $(2.0 \mathrm{~mL})$ at 0 ${ }^{\circ} \mathrm{C}$ under $\mathrm{N}_{2}$, was added dichloroborane-methylsulfide complex $(0.75 \mathrm{~mL}, 6.5 \mathrm{mmol})$ in one portion via syringe. The yellow solution was stirred for $5 \mathrm{~min}$ at $0{ }^{\circ} \mathrm{C}$ then allowed to warm to rt. The solution was stirred for $6 \mathrm{~h}$, and then cannulated into a mixture of $\mathrm{Et}_{2} \mathrm{O}(6 \mathrm{~mL})$ and water $(3$ $\mathrm{mL}$, vigorous bubbling!). This mixture was stirred for $1 \mathrm{~h}$ then poured into a saturated aqueous brine solution $(10 \mathrm{~mL})$. The aqueous layer was extracted with $\mathrm{Et}_{2} \mathrm{O}(1 \mathrm{x} 8 \mathrm{~mL})$ and then with EtOAc $(1 \times 8 \mathrm{~mL})$. The combined organic layers were dried over anhydrous $\mathrm{MgSO}_{4}$, filtered and concentrated to give a pasty off-white solid. The solid was suspended in pentane and the white solid was filtered off. The filtrate was concentrated and allowed to solidify. It was then resuspended in pentane and filtered. The filtrate was reprocessed in this way two more times. In total, $325.5 \mathrm{mg}$ of iv was isolated as a white powder (33\%). TLC analysis showed two spots that correspond to the anhydride and the free acid. The NMR spectra of this material showed a mixture of the monomeric acid as well as the cyclic trimer anhydride. In order to obtain spectra of the monomeric acid, $150 \mu \mathrm{L}$ of water was added to the NMR sample in $\mathrm{CDCl}_{3}$. The biphasic mixture was vigorous shaken then allowed to separate into two layers. Subsequent proton spectra showed only trace amounts of the trimeric material.

Data for iv:

$\mathbf{R}_{f}=0.52$ and 1.0 (40\% EtOAc / Hexanes) - two spots, $\mathrm{KMnO}_{4}$ stain.

IR (KBr pellet): 3462, 3350, 3001, 2956, 2919, 2872, 2849, 1637, 1470, 1425, 1388, 1351, 1152 , $1037,989,923,791 \mathrm{~cm}^{-1}$.

${ }^{1}$ HNMR (500 MHz, $\left.\mathrm{CDCl}_{3}\right)$ : (cyclic trimer) $\delta 6.96(1 \mathrm{H}, \mathrm{td}, J=6.5,17.6 \mathrm{~Hz}, \mathrm{C} 2), 5.53(1 \mathrm{H}, \mathrm{td}, J$ $=1.5,17.6 \mathrm{~Hz}, \mathrm{C} 1), 2.21(2 \mathrm{H}, \mathrm{m}, \mathrm{C} 3), 1.43(2 \mathrm{H}, \mathrm{m}, \mathrm{C} 13), 1.27(18 \mathrm{H}, \mathrm{m}, \mathrm{C} 4-\mathrm{C} 13), 0.88(3 \mathrm{H}, \mathrm{t}, J$ $=6.8 \mathrm{~Hz}, \mathrm{C} 14) ;($ monomeric acid) $\delta 6.52(1 \mathrm{H}, \mathrm{td}, J=6.6,17.9 \mathrm{~Hz}, \mathrm{C} 2), 5.41(1 \mathrm{H}, \mathrm{td}, J=1.6,17.9$ $\mathrm{Hz}, \mathrm{C} 1), 4.36$ (2H, s, -OH), 2.16 (2H, m, C3), 1.41 (2H, m, C13), 1.27 (18H, m, C4-C13), 0.88 $(3 \mathrm{H}, \mathrm{t}, J=6.8 \mathrm{~Hz}, \mathrm{C} 14)$.

${ }^{13}$ CNMR (125 MHz, $\mathrm{CDCl}_{3}$ ): (cyclic trimer) ${ }^{5} \delta$ 157.9, 35.7, 31.9, 29.68, 29.66, 29.64, 29.58, 29.5, 29.4, $29.228 .2,22.3,14.1$.

HRMS (EI+): Calculated for $\mathrm{C}_{42} \mathrm{H}_{81} \mathrm{~B}_{3} \mathrm{O}_{3}\left[\mathrm{M}^{+}\right]$(cyclic trimer): 666.6465 . Found: 666.6481 .

\footnotetext{
${ }^{5}$ The ${ }^{13} \mathrm{C}$ peak for the $\alpha$-carbon is not visible due to the influence of the quadrupolar boron nucleus: Hall, L. W.; Odom, J. D.; Ellis, P. D. J. Am. Chem. Soc. 1975, 97, 4527-4531.
} 

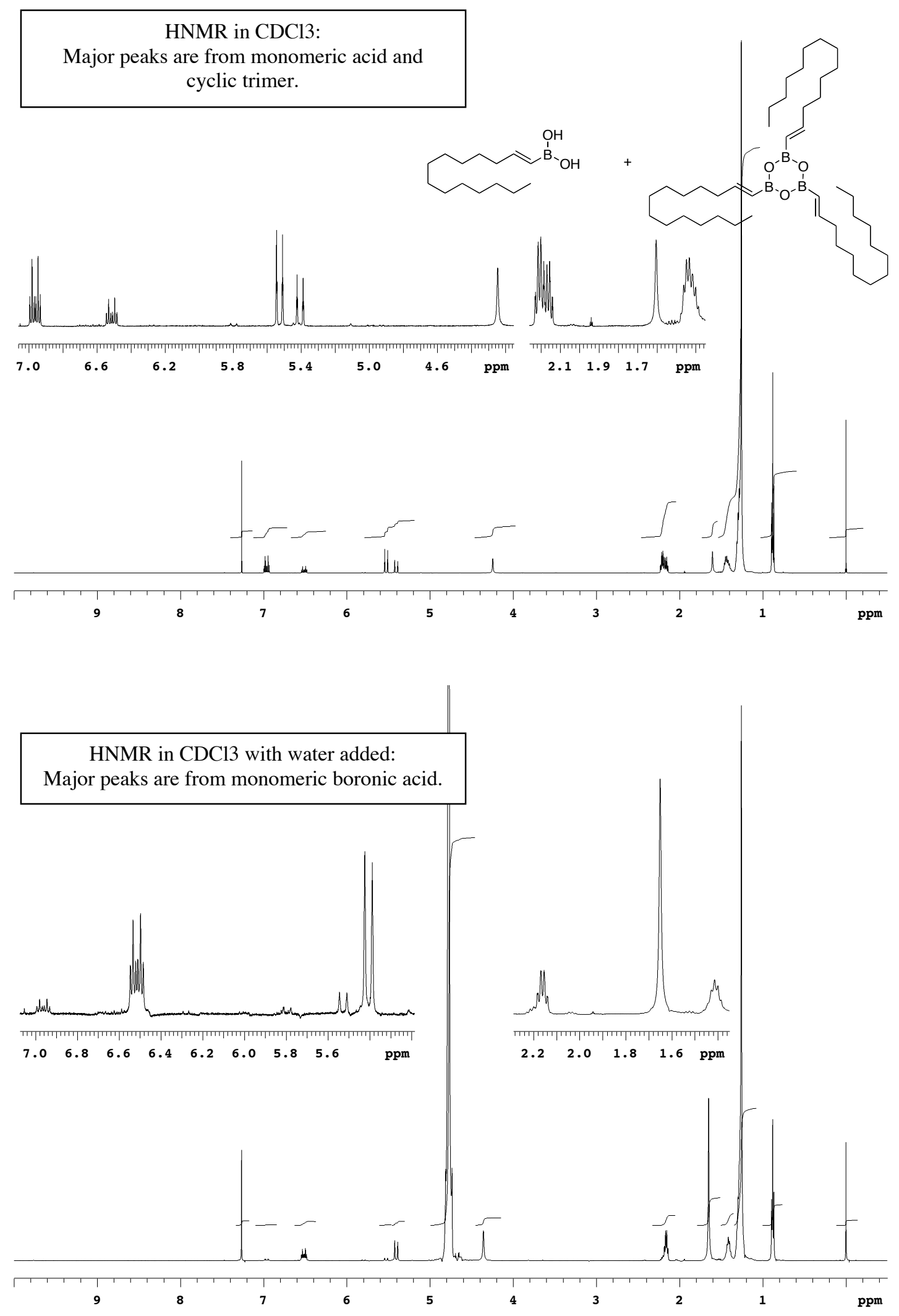


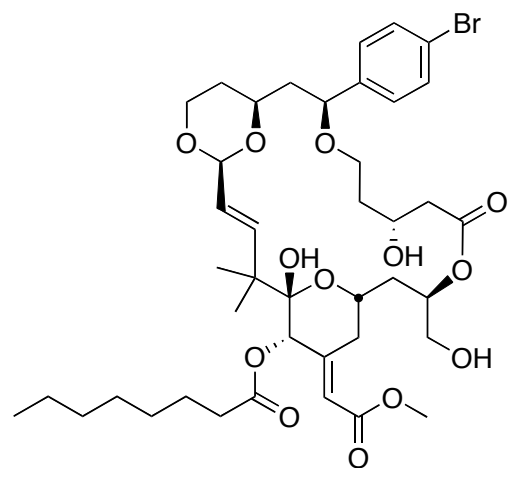

3
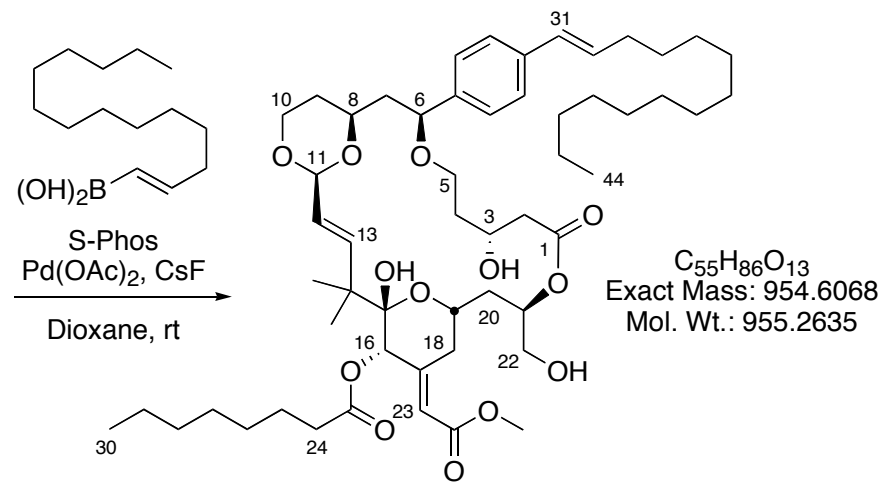

14

Aryl bromide $3(3.0 \mathrm{mg}, 3.6 \mu \mathrm{mol})$ and $\mathrm{CsF}(3.6 \mathrm{mg}, 24 \mu \mathrm{mol})$ were measured into a dry 2 $\mathrm{mL}$ glass vial and placed under high vacuum for $15 \mathrm{~min}$. The vial was removed from the vacuum under $\mathrm{N}_{2}$ gas and placed under an $\mathrm{N}_{2}$ atmosphere. To this vial was added palladium acetate (1.0 $\mathrm{mg}, \quad 4.5 \mu \mathrm{mol}$ ), trans-1-tetradecen-1-ylboronic acid (iv, $1.6 \mathrm{mg}, 7.0 \mu \mathrm{mol}$ ) and 2dicyclohexylphosphino-2',6'-dimethoxy-1,1'-biphenyl (S-Phos, $2.8 \mathrm{mg}, 6.7 \mu \mathrm{mol}$, Strem Chemicals) together as a single portion of a solid mixture. The vial was purged with $\mathrm{N}_{2}$ for 30 sec, then dioxane $(70 \mu \mathrm{L})$ was added via syringe. The rubber septum was removed and the vial was sealed with 2-ply teflon tape, followed by a plastic screw-cap which was finally sealed with parafilm. The vial was vortexed momentarily (to wash solids on the vial walls into the solvent) and the light orange suspension was allowed to stir at $\mathrm{rt}$ for $24 \mathrm{~h}$. The reaction mixture was filtered through a cotton plug using $\mathrm{Et}_{2} \mathrm{O}$ and then concentrated under a stream of $\mathrm{N}_{2}$. Flash chromatography of the residue ( $40 \rightarrow 100 \%$ EtOAc / pentane) yielded $1.2 \mathrm{mg}$ of $\mathbf{1 4}$ (35\%) as well as $1.8 \mathrm{mg}$ of recovered $\mathbf{3}(60 \%)$ both as colorless semi-solid residues (88\% at $40 \%$ conversion).

Data for 14:

$\mathbf{R}_{f}=0.74$ (70\% EtOAc, 30\% pentane) - one black spot $\mathrm{w} / \mathrm{p}$-anisaldehyde stain.

IR (thin film): 3462, 3328 (br), 2924, 2853, 1732, 1374, 1362, 1287, 1258, 1230, 1158, 1135, $1108,1082,1063,1004,980,852,808,755 \mathrm{~cm}^{-1}$.

${ }^{1}$ HNMR (600 MHz, CDCl $)$ : $\delta 7.31(2 \mathrm{H}, \mathrm{m}, \mathrm{Ar}-\mathrm{H}), 7.18(2 \mathrm{H}, \mathrm{m}, \mathrm{Ar}-\mathrm{H}), 6.35(1 \mathrm{H}, \mathrm{dt}, J=15.8$, $1.5 \mathrm{~Hz}, \mathrm{C} 31), 6.23(1 \mathrm{H}, \mathrm{dt}, J=15.9,6.9 \mathrm{~Hz}, \mathrm{C} 32), 6.01(1 \mathrm{H}, \mathrm{d}, J=16.1 \mathrm{~Hz}, \mathrm{C} 13), 6.01(1 \mathrm{H}, \mathrm{d}, J$ $=2.1 \mathrm{~Hz}, \mathrm{C} 23), 5.45(1 \mathrm{H}, \mathrm{dd}, J=7.4,16.0 \mathrm{~Hz}, \mathrm{C} 12), 5.40(1 \mathrm{H}, \mathrm{ddt}, J=5.8,12.2,2.9 \mathrm{~Hz}, \mathrm{C} 21)$, $5.16(1 \mathrm{H}, \mathrm{s}, \mathrm{C} 16), 5.12(1 \mathrm{H}, \mathrm{s}, \mathrm{C} 15-\mathrm{OH}), 5.10(1 \mathrm{H}, \mathrm{d}, J=7.3 \mathrm{~Hz}, \mathrm{C} 11), 4.42(1 \mathrm{H}, \mathrm{d}, J=11.8 \mathrm{~Hz}$, C3-OH), $4.32(1 \mathrm{H}, \mathrm{dd}, J=2.7,11.7 \mathrm{~Hz}, \mathrm{C} 6), 4.28(1 \mathrm{H}, \mathrm{m}, \mathrm{C} 3), 4.08(2 \mathrm{H}, \mathrm{m}, \mathrm{C} 10$ \& C19), 4.03 $(1 \mathrm{H}, \mathrm{ddd}, J=2.7,7.3,11.3 \mathrm{~Hz}, \mathrm{C} 8), 3.91(1 \mathrm{H}, \mathrm{dt}, J=2.2,11.9 \mathrm{~Hz}, \mathrm{C} 10), 3.87(1 \mathrm{H}, \mathrm{ddd}, J=3.1$, 4.9, $11.9 \mathrm{~Hz}, \mathrm{C} 22), 3.72(1 \mathrm{H}, \mathrm{dd}, J=2.6,13.8 \mathrm{~Hz}, \mathrm{C} 18), 3.68$ (3H, s, -OMe), 3.67 (1H, pentet, $J$ $=6.1 \mathrm{~Hz}, \mathrm{C} 22), 3.43(1 \mathrm{H}, \mathrm{ddd}, J=1.7,5.1,9.6 \mathrm{~Hz}, \mathrm{C} 5), 3.29(1 \mathrm{H}, \mathrm{ddd}, J=2.5,9.7,12.8 \mathrm{~Hz}$, C5), $2.57(1 \mathrm{H}, \mathrm{dd}, J=11.6,12.7 \mathrm{~Hz}, \mathrm{C} 2), 2.51(1 \mathrm{H}, \mathrm{dd}, J=2.7,12.7 \mathrm{~Hz}, \mathrm{C} 2), 2.30(2 \mathrm{H}, \mathrm{m}, \mathrm{C} 24)$, $2.23(1 \mathrm{H}, \mathrm{m}, \mathrm{C} 4), 2.21(1 \mathrm{H}, \mathrm{dd}, J=1.3,7.1 \mathrm{~Hz}, \mathrm{C} 33), 2.18(1 \mathrm{H}, \mathrm{dd}, J=1.3,6.8 \mathrm{~Hz}, \mathrm{C} 33), 2.13$ $(1 \mathrm{H}, \mathrm{ddd}, J=7.5,11.8,15.8 \mathrm{~Hz}, \mathrm{C} 7), 2.06(1 \mathrm{H}, \mathrm{ddd}, J=1.9,11.5,13.6 \mathrm{~Hz}, \mathrm{C} 18), 2.01(1 \mathrm{H}$, ddd, $J=2.6,12.5,14.3 \mathrm{~Hz}, \mathrm{C} 20), 1.99(1 \mathrm{H}, \mathrm{dd}, J=4.8,6.8 \mathrm{~Hz}, \mathrm{C} 22-\mathrm{OH}), 1.81(1 \mathrm{H}$, ddd, $J=2.9,11.5$, $14.2 \mathrm{~Hz}, \mathrm{C} 20), 1.75(1 \mathrm{H}, \mathrm{dq}, J=4.8,12.5 \mathrm{~Hz}, \mathrm{C} 9), 1.60(3 \mathrm{H}, \mathrm{m}, \mathrm{C} 7$ \& C25), $1.45(3 \mathrm{H}, \mathrm{m}, \mathrm{C} 4$ \& C34), $1.38(1 \mathrm{H}, \mathrm{d}$ (br), $J=13.4 \mathrm{~Hz}, \mathrm{C} 9), 1.21-1.36(26 \mathrm{H}, \mathrm{m}, \mathrm{C} 26-\mathrm{C} 29$ \& 35-43), $1.20(3 \mathrm{H}, \mathrm{s},-$ $\left.\mathrm{C}\left(\mathrm{CH}_{3}\right)_{2}\right), 1.05\left(3 \mathrm{H}, \mathrm{s},-\mathrm{C}\left(\mathrm{CH}_{3}\right)_{2}\right), 0.88(3 \mathrm{H}, \mathrm{t}, J=7.0 \mathrm{~Hz}, \mathrm{C} 30$ or $\mathrm{C} 44), 0.87(3 \mathrm{H}, \mathrm{t}, J=7.1 \mathrm{~Hz}$, $\mathrm{C} 30$ or $\mathrm{C} 44)$.

${ }^{13}$ CNMR $\left(125 \mathrm{MHz}, \mathrm{CDCl}_{3}\right): \delta 172.5,172.1,167.0,151.6,142.5,139.5,137.9,131.7,129.1$, 126.6 (2C), 126.1 (2C), 126.0, 119.9, 102.3, 98.9, 83.8, 75.9, 74.1, 71.6, 68.8, 66.3, 65.7, 65.6, 
$64.5,51.1,45.4,45.1,42.1,35.9,34.6,33.6,33.0,32.3,31.9,31.6,31.0,29.67,29.66,29.63$, 29.61, 29.5, 29.3 (2C), 29.2, 29.0, 28.9, 24.7, 24.3, 22.7, 22.6, 19.4, 14.12, 14.06.

HRMS (MALDI): Calculated for $\mathrm{C}_{55} \mathrm{H}_{86} \mathrm{O}_{13} \mathrm{Na}$ [M+Na]: 977.5961. Found: 977.5923.

$[\alpha]_{D}^{24}=-62.6^{\circ}\left(c=0.16, \mathrm{CHCl}_{3}\right)$. 

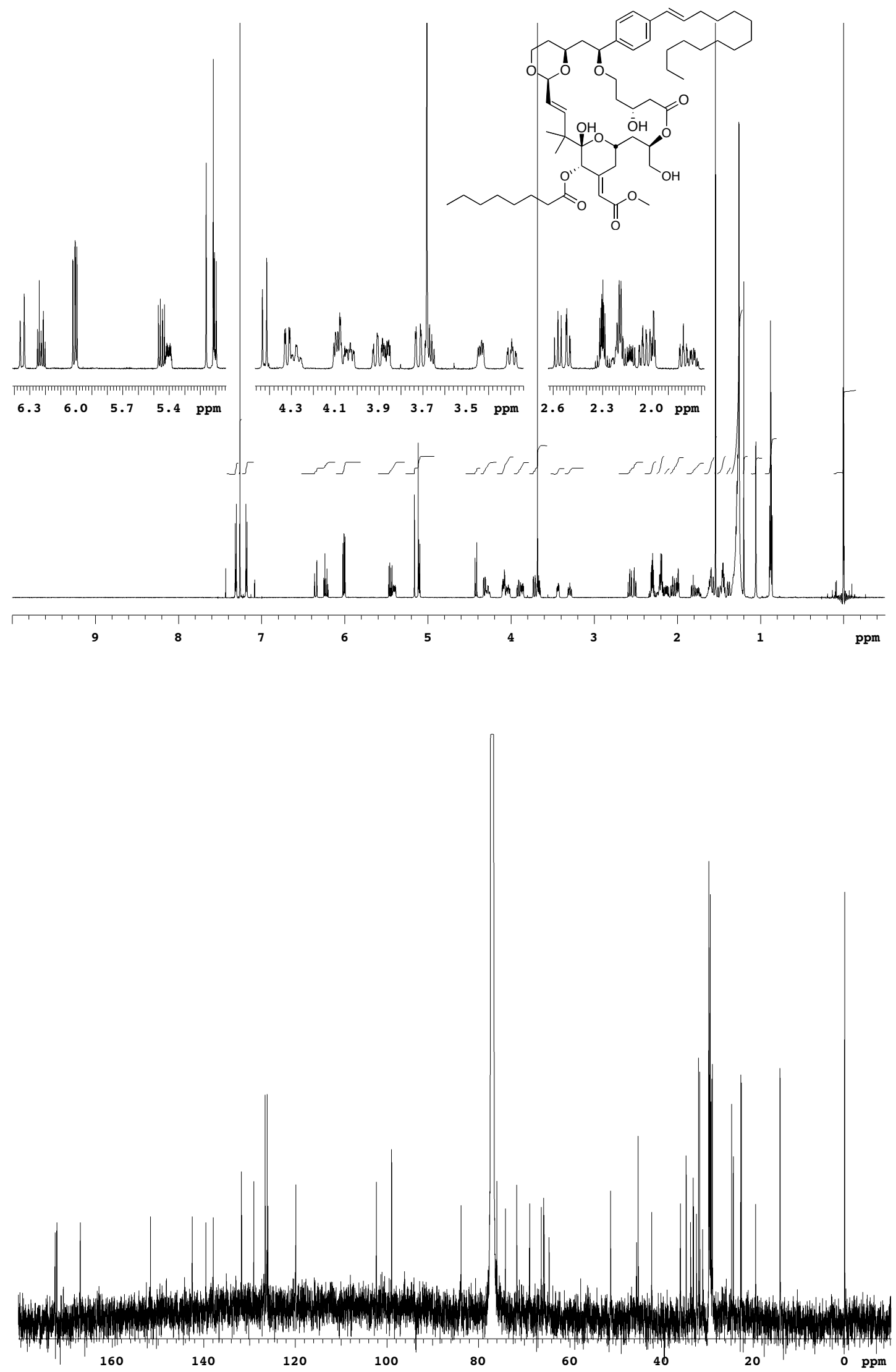